\title{
GCU
}

Glasgow Caledonian

University

University for the Common Good

\section{Atomic spectrometry update. Review of advances in the analysis of metals, chemicals and materials}

Carter, Simon ; Fisher, Andy S.; Hinds, Michael W.; Lancaster, Steve; Marshall, John

Published in:

Journal of Analytical Atomic Spectrometry

DOI:

10.1039/c3ja90051g

Publication date:

2013

Document Version

Publisher's PDF, also known as Version of record

Link to publication in ResearchOnline

Citation for published version (Harvard):

Carter, S, Fisher, AS, Hinds, MW, Lancaster, S \& Marshall, J 2013, 'Atomic spectrometry update. Review of advances in the analysis of metals, chemicals and materials', Journal of Analytical Atomic Spectrometry, vol. 28, no. 12, pp. 1814-1869. https://doi.org/10.1039/c3ja90051g

\section{General rights}

Copyright and moral rights for the publications made accessible in the public portal are retained by the authors and/or other copyright owners and it is a condition of accessing publications that users recognise and abide by the legal requirements associated with these rights.

Take down policy

If you believe that this document breaches copyright please view our takedown policy at https://edshare.gcu.ac.uk/id/eprint/5179 for details

of how to contact us. 


\section{ASU REVIEW}

Cite this: J. Anal. At. Spectrom., 2013, 28, 1814

Received 3rd September 2013 Accepted 3rd September 2013

DOI: 10.1039/c3ja90051g

www.rsc.org/jaas

\section{Atomic spectrometry update. Review of advances in the analysis of metals, chemicals and materials}

\author{
Simon Carter, ${ }^{a}$ Andy S. Fisher, ${ }^{\mathrm{b}}$ Michael W. Hinds, ${ }^{\mathrm{c}}$ Steve Lancaster ${ }^{\star d}$ \\ and John Marshalle
}

\section{$1 \quad$ Metals}

1.1 Ferrous metals

1.2

2

2.1

2.1.1

2.1.2

2.1.3

2.1.4

2.2

2.2.1

2.2.2

2.2.3

2.2.4

2.2.5

2.3

2.3.1

2.3.2

2.3.3

2.3.4

2.3.5

2.4

2.4.1

2.4.2

2.4.3

3

3.1 art objects

Speciation dissolution materials

Materials
Non-ferrous metals

Industrial chemicals

Fuels and lubricants

Petroleum products-gasoline, diesel, gasohol and exhaust particulates

Fuel-coal, peat and other solid fuels

Oils-crude oil, lubricants

Alternative fuels

Organic chemicals and solvents

The analysis of archaeological, cultural heritage and

Remote analysis of harmful materials

Applications requiring minimal sample preparation Applications requiring analyte extraction or sample

Inorganic chemicals and acids

Coupled techniques

Forensic applications

Remote analyses of inorganic materials

Other applications of the analysis of inorganic

Analysis of nano-structures

Nuclear materials

Reviews, overviews and CRMs

Nuclear safeguards and forensics

Other nuclear applications

Ceramics and refractories
3.1.1

3.2

3.2.1

3.2 .2

3.3

3.3.1

3.3.2

3.3.3

3.4

3.4.1

3.4.2

3.4.3
Analysis of archaeological, cultural heritage and art objects

Thin Films and Depth Profiling

Laser-based techniques

Glow discharge and plasma techniques

Glasses

Laser-based techniques

Other techniques

Analysis of archaeological, cultural heritage and art objects

Catalysts

Surface Characterisation

Sampling and sample preparation

Miscellaneous

This review period has seen some changes to the format of the review. These changes are intended to more accurately reflect the current state of analytical research in the analysis of metals, chemicals and materials. As a consequence, the title of the review and some of the section headings have been altered to reflect this. Significant areas of growth include the use of LIBS in remote analysis, especially of explosives and nuclear materials. The standoff capability of the technique makes it very desirable in these areas. The use of chemometrics and procedures for removing substrate interferences are proving to be effective in making the technique more quantitative. It is also beginning to be reported for use in Process Analysis in the pharmaceuticals industry and has been employed to investigate the processes occurring within an engine and to model the processes occurring in explosions. The analysis of archaeological, cultural heritage and art objects continues to be a significant area of research, with some interesting developments in multi-analytical techniques which are employed to yield maximum information with as little disruption to the object as possible. Similarly, the high resolution capabilities of synchrotron radiation are being increasingly employed for high value objects where the cost of the analysis is outweighed by the benefits. In the field of materials analysis, the development of 
techniques for investigating the growth of novel material structures is a significant activity, e.g., the nucleation of alpha Alumina via titania templates, studied using a range of techniques. Papers outlining advances in the use of asymmetric field flow fractionation with a multi-detection approach for the characterisation of particle size and size distribution are reviewed as is a comparison of hydrodynamic chromatography with AFFF. Papers dealing with single particle analysis are also reviewed. The catalyst section is shorter than in previous reviews and this reflects the fact that fewer new developments in analytical chemistry are being published. The emphasis is on advances in the analysis of catalyst materials. This is the latest review covering advances in atomic spectrometric measurements of metals, chemicals and materials. It follows on from last year's review ${ }^{1}$ and should be read in conjunction with other reviews in the series. ${ }^{2-5}$ This year will be the last time that Mike Hinds will write for the review. His contribution over many years has been very significant and greatly appreciated. We welcome John Marshall to the writing team.

\section{Metals}

For all types of metals and alloys, the most interesting contributions continue to centre on solid sample analysis methods. There continues to be a large number of papers in which different combinations of spectroscopic and non-spectroscopic methods were applied to the problem of interest. The overall distribution of papers reviewed for the metals section (as percentage of papers received) by instrumental method was: atomic absorption 3\%, optical emission 25\% (LIBS included at $21 \%$ ), mass spectrometry 9\%, SIMS 8\%, XRF 13\%, and the use of multiple instrumental methods $40 \%$. Papers that are fundamental in nature and/or focus on instrumental development were not cited. A number of papers were included in Table 1 which demonstrated a range of materials and/or analytes that were thought noteworthy.

\subsection{Ferrous metals}

A large number of researchers have used multiple spectroscopic methods along with other analytical methods to study metals. The phases of three aluminium-iron-niobium alloys determined using XRD and subjected to electrochemical treatment for dealloying were described in a paper by Drensler et al. ${ }^{6}$ The preferential dissolution of Fe was determined in the electrolyte using FAAS and confirmed using SEM-EDXRF analysis of the remaining alloy. This study showed that phases in the alloys depend on how the alloys were prepared and heat treated. The oxidation of $\mathrm{Zr}$ doped iron-chromium-aluminium-ytterbium alloy thin walled heating elements was examined under a variety of experimental conditions using thermogravimetry, optical microscopy, SEM, TEM, sputtered neutrals mass spectrometry (SNMS) and GD-OES by Gopalakrishnan et al. ${ }^{7}$ Increased emissivity of the alumina scales formed on the alloy can be obtained by suitable Zr-doping of the alloy. Mun et al. ${ }^{8}$ studied B segregation to austenite grain boundaries in low alloy steel, by a combination of SIMS and particle tracking autoradiography (PAT). The segregation was examined both during cooling and when the alloy was held at one temperature. The distribution of $\mathrm{B}$ indicated that the retardation effect of $\mathrm{B}$ on ferrite transformation was due to the cooling rate dependence of $\mathrm{B}$ segregation into the austenite grain boundaries during cooling. Another group, Kim et al., ${ }^{9}$ also used SIMS and PAT to estimate the distribution of B in the coarse grained heat affected zone of low alloy steels. Steel containing $30 \mu \mathrm{g} \mathrm{g}^{-1} \mathrm{~B}$ showed high segregation of B even at low heat inputs whereas the opposite was observed when B concentration was lower at $10 \mu \mathrm{g} \mathrm{g}^{-1}$.

The nano-structure and nano-chemical composition of dissimilar weld joints between nickel-based alloy, Alloy 690, and low alloy steel were studied by using optical microscopy, SEM, TEM, SIMS, and 3D atom probe tomography as reported by Choi et al. ${ }^{10}$ Examination of the weld root region revealed an unmixed zone in the nickel-based alloy, a fusion boundary and a heat affected zone in the low alloy steel. Small scale analysis of the low alloy steel indicated a non-homogeneous distribution of elements with higher $\mathrm{Fe}$ and lower concentrations of $\mathrm{Cr}, \mathrm{Mn}$ and $\mathrm{Ni}$. It was also observed that carbides precipitated near the fusion boundary. Kaciulis et al. ${ }^{11}$ examined the microstructure and microchemistry of nickel-based super alloys with biphasic structure (gamma and gamma' phases), using SEM, AFS, PIXE and scanning photoemission microscopy (SPEM). The compositional differences between the two phases were elucidated through SPEM spectral images. The development of the detrimental gamma' phase in the alloy, was due to both external applied stress and to diffusion of $\mathrm{W}$ from the gamma to gamma' phase. Rafique et al. ${ }^{12}$ synthesized 3D flower-like iron-cobalt alloy nanostructures and characterized them using a variety of instrumental methods. The iron-cobalt crystalline structure, with an average crystal size of $24 \mathrm{~nm}$, was determined using XRD. The nearly equi-atomic composition (FeCo) was confirmed by using EDXRF and ICP-OES. It was also found that the flower-like structure was composed of six nano triangular prisms. The base and height dimensions that range between 100 and $200 \mathrm{~nm}$ were determined through the use of SEM and TEM.

A variety of methods are applicable to examining metal coatings. Itani et al. ${ }^{13}$ studied the properties of two types of zincchromium coatings on steel using XPS and scanning Auger electron spectroscopy. Specific regions of interest were examined: top surface, shallow subsurface and the whole bulk to the steel interface. Elemental composition was determined using XPS and compounds were identified by Auger electron spectrometry by comparison to reference samples and materials. It was observed that one type of coating was composed of metallic zinc and chromium with oxides residing only on the surface and interface. The other coating was composed mainly of zinc and chromium oxides. Thin coatings of titanium carbonitride on steel were studied by Miller et al. ${ }^{14}$ using Auger electron spectroscopy to confirm the composition of the coating. The chemical state of the elements in the coating was determined using XPS and the morphology of the coating was investigated using atomic force microscopy. In air, the coatings were oxidized but maintained their uniformity as indicated by 
Table 1

Technique; Atomization;

Element

$\mathrm{Ag}$ and $\mathrm{Au}$

Matrix

Copper

Presentation

$\mathrm{AA} ; \mathrm{F} ; \mathrm{L}$

$\mathrm{Al}$

$\mathrm{Al}$ and $\mathrm{Zn}$

$\mathrm{Cr}$

Pt

Ta

Various(4)

Various

Various (7)
Galvanized steel

Uranium-tantalum alloy

Aluminum-magnesiumsilicon

Titanium-6aluminumvanadium alloy

Magnesium

Tool steel

Tantalum

SIMS;一; S

XRF;一; S

XRF;一; S

OES; GD; S

OES; ICP; L
Comments

Ref.

Blister copper was dissolved

24

in nitric acid. The solution

was filtered and the trapped residue was solubilized in

aqua regia (1 part $\mathrm{HNO}_{3}+3$

parts $\mathrm{HCl}$ ). Both the

supernatant solution and

the re-dissolved portions

were determined for Au and

Ag by FAAS. There was good

agreement with results from

fire assay reported.

The diffusion of $\mathrm{Al}$ into

magnesium was studied

through SIMS depth

profiling.

The selective dissolution of

$\mathrm{Al}$ and $\mathrm{Zn}$ by varying the $\mathrm{pH}$

from 2 to 12 in $30 \mathrm{mM} \mathrm{NaCl}$

followed by ICP-OES analysis

of the resulting solutions.

Dissolution of $\mathrm{Zn}$ occurred

at low $\mathrm{pH}$ whereas $\mathrm{Al}$

dissolved at high $\mathrm{pH}$.

LIBS was used to follow the

real time deposition of $\mathrm{Cr}$

onto H13 Tool Steel.

Accuracy was assessed to be within $2.78 \%$.

SIMS was used to determine extent of Pt implanted into titanium through depth

profile measurements.

Concentration of $\mathrm{Ta}$ in the

alloy was determined by

EDXRF. RSD's were reported as $0.6 \%$ for $1.5 \% \mathrm{Ta}$ and

$0.3 \%$ for $5 \%$ Ta.

The solubility of Fe in molten alloy was examined by sampling the melt and analysing the solidified material.

The effects of surface laser treatment were analysed by elemental depth profiling by GD-OES.

Titanium alloy samples were dissolved in a mixture of $\mathrm{HF}$ and $\mathrm{HNO}_{3}$ at $70^{\circ} \mathrm{C}$. The limit of detection was estimated to be between 10 and $27 \mu \mathrm{g}$ $\mathrm{L}^{-1}$ for the suite of elements. Recovery study results were between 95 and 105\%. An RSD value was quoted but without a concentration to give it meaning. 
Table 1 (Contd.)

\begin{tabular}{|c|c|c|c|c|}
\hline Element & Matrix & $\begin{array}{l}\text { Technique; Atomization; } \\
\text { Presentation }\end{array}$ & Comments & Ref. \\
\hline Various (16) & Uranium-zirconium alloy & OES; ICP; L & $\begin{array}{l}\text { The alloy sample was } \\
\text { dissolved and the alloy } \\
\text { metals were extracted into } \\
\text { an organic phase consisting } \\
\text { of } 30 \% \text { tributyl phosphate in } \\
\text { carbon tetrachloride, leaving } \\
\text { the analyte elements in the } \\
\text { aqueous phase. }\end{array}$ & 33 \\
\hline Various (5) & Zirconium & AA; ETA; L & $\begin{array}{l}\text { Zirconium was dissolved } \\
\text { and trace elements were } \\
\text { determined by GF-AAS. } \\
\text { Detection limits in solid } \\
\text { were estimated to be } 0.05 \text {, } \\
1.0,0.5,1.0, \text { and } 5.0 \mu \mathrm{g} \mathrm{g}^{-1} \\
\text { for Cd, Co, Cu, Mn, and } \mathrm{Zn} \text {, } \\
\text { respectively. }\end{array}$ & 34 \\
\hline
\end{tabular}

TOF-SIMS. Mechanical tests were also done which compared the coating with the underlying steel substrate.

The study of metal corrosion has been a topic of interest during this review period. A selective, quantitative, and sensitive method for the determination of $\mathrm{Cr}^{\mathrm{VI}}$ in corrosion protection coatings for hard chrome coatings on copper or zinc electro-

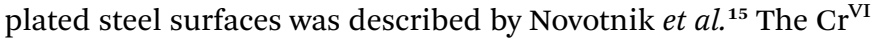
species was determined by ultrasonic extraction with an alkaline solution ( $\mathrm{pH}$ 12), followed by anion-exchange HPLC-ICPMS. Importantly, the interconversion of species was measured by using enriched isotope solutions of ${ }^{50} \mathrm{Cr}^{\mathrm{VI}}$ and ${ }^{53} \mathrm{Cr}^{\mathrm{III}}$. The method accuracy was validated by measuring $\mathrm{Cr}^{\mathrm{VI}}$ in BCR CRM 545 welding dust, with both external calibration and speciated

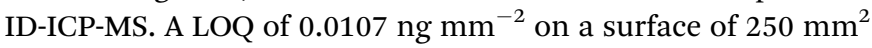
was reported, however the authors did not specify the accepted RSD for this claim. Corrosion products of a 450 year old iron nail were investigated at the nanometer level using synchrotron based scanning transmission X-ray microscopy (STXM) as reported by Michelin et al. ${ }^{\mathbf{1 6}}$ Data acquired from a thin film of the metal-corrosion products, including the interface, showed an interfacial layer of $100 \mathrm{~nm}$ of maghemite and magnetite (as compared with iron mineral reference spectra). Further from the interface, the corrosion products were mainly iron carbonates and smaller quantities of iron oxides. Although the authors claim to have insights into the long term corrosion of steels, it must be pointed out that as the actual material was iron, corrosion is strongly related to the environmental conditions.

Groenewold et al. ${ }^{17}$ used a number of methods to characterize arsenic contamination in rust from steel containers that held chemical warfare agents including 2-chlorovinyldichloroarsine. The distribution and state of As in rust was examined by using SIMS, XPS, Auger electron spectroscopy, and SEM-EDXRF. At the topmost layer, $\mathrm{As}^{\mathrm{III}}$ and $\mathrm{As}^{\mathrm{V}}$ were homogeneously distributed and closely associated with iron. Studies with XPS and SIMS showed As in the reduced state at depths of several nanometers. The SEM-EDXRF observations indicated that As was present at depths of several microns. It was not homogeneously distributed and may be in different chemical states and concentrations. Rust must be completely removed from these steel containers to ensure they are free of arsenic. The oxidation of nickel-based super alloys was studied by Kim et al. ${ }^{18}$ by using LIBS to do elemental depth profiling. A complex outer layer consisting of $\mathrm{MnCr}_{2} \mathrm{O}_{4}$ and $\mathrm{Cr}_{2} \mathrm{O}_{3}$ was found along with an inner layer of $\mathrm{Al}_{2} \mathrm{O}_{3}$ from both Alloy 617 and Haynes 230 super alloys. The authors claim depth profiles obtained by LIBS were in broad agreement with profiles obtained using SIMS, SEM-EDXRF and XRD. However, SEM-EDXRF and XRD are usually not able to do true depth profiling.

Elemental mapping of an engine valve using LIBS demonstrated the capability of the method to characterize complex shapes, as described by Lopez-Quintas et al. ${ }^{19}$ Chemical maps of $\mathrm{Cr}, \mathrm{Fe}, \mathrm{Mn}$ and $\mathrm{Ni}$ were determined. Depth profiles at different sites on the engine valve were made for $\mathrm{Cr}$ and Fe. The authors suggested that a LIBS instrument with this capability would be a useful evaluation tool for the quality control of manufactured metal parts. A LIBS system has been designed for fast analysis and sorting of metallic scrap pieces as described by Gurell et al. ${ }^{\mathbf{2 0}}$ The laser operated at a distance of 1 meter from the samples and 5-10 pulses (at $20 \mathrm{~mJ}$ ) bored through to the bulk material. The apparatus successfully sorted eighteen different certified reference materials (CRMs) in the laboratory and will be tested in a factory. Gonzaga and Pasquini ${ }^{21}$ described a compact and low cost LIBS instrument comprising a diode pumped Q-switched neodymium:lanthanum scandium borate (Nd:LSB) microchip laser, a Czerny-Turner spectrograph (250-390 nm) and a 1024 pixel linear array detector. This spectrometer was used to determine $\mathrm{Cr}$ and $\mathrm{Ni}$ in steels in which the content of these elements ranged from 5 to $26 \%$. The relative errors for these elements were $3.7 \%$ for $\mathrm{Cr}$ and $6.7 \%$ for $\mathrm{Ni}$; the authors claim that the errors were similar to, or lower than, other more costly LIBS analysers. This paper is mentioned in this review because continued innovation toward more compact systems could eventually lead to a handheld LIBS analyser. 
It has been reported by Lefaix-Jeuland et al. ${ }^{22}$ that SIMS was very effective when used for measuring the distribution of $\mathrm{He}$ implanted into mono and poly crystalline iron samples. Primary $\mathrm{Cs}^{+}$ions combined with $\mathrm{He}$ to form detectable $\mathrm{CsHe}^{+}$ions which allowed concentration levels of He above $60 \mu \mathrm{g} \mathrm{g}^{-1}$ to be measured. Implanted He was detected to a depth of $220 \mathrm{~nm}$ which was in agreement with predicted values.

Wang et al. ${ }^{23}$ used SR-XAS to identify and quantitate zinc speciation in zinc-containing wastes from steelmaking. Dust collected from the electric arc furnace and the basic oxygen furnace contained primarily $\mathrm{ZnFe}_{2} \mathrm{O}_{4}$. Zinc in the off gas sludge from the basic oxygen furnace was present as $\mathrm{Zn}_{5}\left(\mathrm{CO}_{3}\right)_{2}(\mathrm{OH})_{6}$, $\mathrm{ZnFe}_{2} \mathrm{O}_{4}$, and $\mathrm{ZnSO}_{4} \cdot 7 \mathrm{H}_{2} \mathrm{O}$. Blast furnace dust was comprised of $39 \% \mathrm{ZnCl}_{2}$ (soluble phase) and $61 \% \mathrm{ZnFe}_{2} \mathrm{O}_{4}$ (insoluble phase). Similarly, $\mathrm{ZnCl}_{2}$ was found in the insoluble phase of blast furnace sludge but the soluble fraction appeared to contain a zinc silicate.

\subsection{Non-ferrous metals}

As in the previous section, multiple spectroscopic methods are becoming increasingly important and a number of papers dealing with this area are reviewed. The morphology, element content, porosity and pore diameter distribution in foam aluminium were characterized using SEM, ICP-MS and a mercury injection apparatus as reported by Wang and Ren. ${ }^{35}$ Samples were observed to have uniform pore sizes and changes to the particle size of $\mathrm{NaCl}$ used in the manufacturing process resulted in similar changes to the foam aluminium pore sizes Oztoprak et al. ${ }^{36}$ investigated two copper plates welded together with stellite 6 powder (cobalt based alloy) using a millisecond pulse from a Nd:YAG laser. The composition of the weld and diffusion of the stellite 6 into the copper plates were followed using LIBS through depth profiling. Results from SEM-EDXRF showed that stellite 6 particles penetrate into the copper plates and mechanical tests indicated that this process increased hardness of the material. Holmberg et $a .^{37}$ characterized the brightly coloured uniform passive layers formed on zirconium by electrochemical treatments using many different methods. Profilometry and AFS were used to examine the surface roughness and surface morphology. Electron backscattering diffraction and SEM measurements showed that the passive coloured layers were uniform and crystalline. The corrosion products in $1 \% \mathrm{NaCl}$ solutions were determined using ICP-MS. There was little corrosion when the samples were polished, whereas etched samples showed substantially more corrosion. The effect of solidification parameters on the microstructure of antimonybismuth-zinc lead-free solder was described by Cadirli et al., ${ }^{38}$ who used XRF to determine the composition of the microstructure. The phases of the microstructure were identified through the use of XRD. The combination of XRF and XRD was also applied to the analysis of nano-carbide transformation on used $2.25 \mathrm{Cr}-1 \mathrm{Mo}$ boiler tubes. ${ }^{39}$ Measurements by XRD identified the carbide-phase components and an increase in the carbide phase, with tube age, was observed. A decrease in Mo concentration with ageing in the carbide phase region was determined using XRF.
The combination of Raman spectroscopy and XRF was used in three different applications. Sutter et al. ${ }^{\mathbf{4 0}}$ studied the surfaces of a titanium-aluminium-vanadium alloy and tantalum after sliding across each other (at $34 \mathrm{~m} \mathrm{~s}^{-1}$ ) using the techniques of Raman spectroscopy and XRF. The presence of $\mathrm{Ti}_{2} \mathrm{O}$ in anatase form confirms a rapid and a very localized rise in temperature on the friction surface. There was also evidence of titanium alloy transferring material onto the surface of tantalum (less than $10 \mu \mathrm{m}$ thickness) despite the high sliding velocity. Surface roughness was also studied using a profilometer. The second application, by Petrova et al., ${ }^{\mathbf{4 1}}$ examined the gemstones and noble metals in a sceptre. A handheld Raman spectrometer was used to confirm types of gemstones attached to the sceptre. The presence of silver alloys and gilding were identified by a handheld XRF spectrometer. The silver values from semiquantitative analysis were confirmed by hallmarks and newly discovered documentation. The authors concluded that the combination of Raman and XRF handheld spectrometers is an ideal tool for studying historical artefacts where in situ investigations are necessary. Colomban et al. ${ }^{\mathbf{4 2}}$ also used portable Raman and $a$ hand held XRF instrument to analyse Asian bronze and brass pieces in situ at a museum. The composition of the metal alloys was determined using XRF and Raman spectroscopy was used in an to attempt to identify the compounds making up the patina on these metal surfaces in a non-invasive manner. The following compounds were identified in the patina: $\mathrm{CuO}, \mathrm{Cu}_{2} \mathrm{O}$, $\mathrm{Cu}_{2} \mathrm{~S}$, tin oxides, $\mathrm{ZnO}, \mathrm{Cu}_{3}(\mathrm{OH})_{4} \mathrm{SO}_{4}, \mathrm{Cu}_{4}(\mathrm{OH})_{6} \mathrm{SO}_{4}, 3 \mathrm{PbOPb}-$ $\mathrm{SO}_{4} \cdot \mathrm{H}_{2} \mathrm{O}, \mathrm{PbSO}_{4} / \mathrm{PbO}, \mathrm{Pb}\left(\mathrm{AsO}_{4}\right)_{3} \mathrm{Cl}$ and $\mathrm{HgO} / \mathrm{HgS}$. It is interesting to note that although worldwide sales of hand held XRF spectrometers have been quite high (reviewer's informal discussions with XRF vendors), there were relatively few reports in the literature in this review period related to the use of these portable instruments.

The surface characteristics titanium sandblasted with zirconia particles were compared with the surface effects using conventional alumina particles in order to improve osseointegration for titanium bone implants. Li et al. ${ }^{43}$ used SEM, profilometry and XRF, to study these surfaces. There were fewer residual zirconia particles remaining on the titanium surface $(0.13 \pm 0.07 \%)$ than were observed for alumina $(6.74 \pm 0.74 \%)$. However, the alumina particles generated a rougher surface than zirconia particles under the same experimental conditions. Overall, the authors concluded that zirconia particles were preferred over alumina because zirconia is more biocompatible than alumina, fewer zirconia particles remain on the surface after sandblasting, and sufficient surface roughness was obtained for osseointegration. Titanium alloys manufactured for biomedical implant applications were surface modified by laser treatment, as outlined by Chikarakara et al. ${ }^{\mathbf{4 4}}$ Metallography tests showed martensite structure formed on the laser treated region and EDXRF analysis indicated a more homogeneous element distribution after laser treatment. There was also a $67 \%$ increase in microhardness after laser treatment compared with the bulk material.

Multiple methods were also applied to metal art objects and metal archaeological pieces. The surface structure of a silver coin, manufactured in the Japanese Edo period, was studied using 
XRD, EDXRF, electron diffraction, SEM and TEM as described in a paper by Taguchi and Kirino. ${ }^{45}$ Results obtained using EDXRF showed a composition of $13 \% \mathrm{Ag}$ and $87 \% \mathrm{Cu}$. However, reflectance spectra did not show evidence of $\mathrm{Cu}$. Polishing some coins revealed that the coins changed to a copper colour and reflectance spectra showed the presence of $\mathrm{Cu}$. The microstructure analysed using TEM showed that a related silver coin had two surface layers: a $0.6 \mu \mathrm{m} \mathrm{Ag}$ rich layer over top of a 0.8 $\mu \mathrm{m}$ thick layer of $\mathrm{Cu}_{2} \mathrm{O}$. This suggested that the surface of the coin had been treated to form a silver layer. Pardini et al. ${ }^{46}$ used XRF and LIBS to determine the elemental composition of a large collection of silver Roman republican denarii coins encompassing a time period of about two centuries. Most of the measurements were performed in situ at the Monetiere museum in Florence. There was a remarkable connection between the quality of the silver and some crucial contemporary events. A group of 416 silver coins from the Ottoman Empire (16th and 17th centuries) were analysed to Rodrigues et al. ${ }^{47}$ to confirm the fineness of silver and also to study the provenance of the alloys used in the manufacture of these coins. Small cross sections of the coins were cut, embedded in plastic and analysed using SR- $\mu$ XRF and $\mu$ PIXE. The silver content ranged between $90 \%$ and $95 \%$. These measurements did not agree with the historical interpretations which predicted a debasement of approximately $44 \%$ of the silver content in the coins. This might have been a very well preserved batch of coins and, therefore, did not represent the general population of these silver coins.

Gouda et al. ${ }^{48}$ determined the chemical composition of ten ancient Egyptian bronze statues using XRF spectrometry and used XRD to study the corrosion products and composition of eleven damaged artefacts. The damaged artefacts were composed of a lead bronze alloy where the $\mathrm{Pb}$ concentration varied from 3.43 to $18.04 \%$ and the Sn varied from 2.53 to $10.67 \%$. For eight of the eleven artefacts, the patina was mainly composed of cuprite $\left(\mathrm{Cu}_{2} \mathrm{O}\right)$ along with other compounds such as cassiterite $\left(\mathrm{SnO}_{2}\right)$, cerussite $\left(\mathrm{PbCO}_{3}\right)$, atacamite $\left(\mathrm{Cu}_{2}(\mathrm{OH})_{3} \mathrm{Cl}\right)$ and paratacamite $\left(\mathrm{Cu}(\mathrm{OH})_{3} \mathrm{Cl}\right)$.

There is continued interest in the application of LIBS to various metal investigations. Galbacs et al. ${ }^{49}$ compared results obtained using a multiple pulse LIBS system with those obtained using ICP-OES and ICP-MS for the determination of $\mathrm{Pb}$ and $\mathrm{Sn}$ in soldering tin samples. It was found that the concentration range of multi-pulse LIBS was broader than that from single pulse LIBS. The accuracy demonstrated by the LIBS method was $1-5 \%$, although the authors did not specify whether this was relative accuracy or absolute accuracy. They further claimed that LIBS was sufficient for semiquantitative purposes in this application and that it provides rapid in situ analyses that would be useful for soldering alloy discrimination.

The application of LIBS to precious metal recovery and recycling of precious metal scrap was described by Legnaioli et al. ${ }^{50}$ The authors presented evidence to support their claim that LIBS would be a viable alternative to ICP-OES and XRF. The LOD, for the elements considered, was in the order of $0.2 \mathrm{mg} \mathrm{g}^{-1}$ and a relative standard deviation of $5 \%$ was observed for Au at $20 \mathrm{mg} \mathrm{g}^{-1}$.

Suyanto et al. ${ }^{51}$ quantitatively determined deuterium in zircaloy with a LIBS system designed without a sample chamber but with the sample in a helium gas environment. The LOD was estimated to be $20 \mu \mathrm{g} \mathrm{g}^{-1}$ and with only a $10 \mu \mathrm{m}$ diameter crater produced by the laser pulses required for the measurement. It was noted that this method had potential to be used as a routine, in situ and virtually non-destructive method for determining deuterium in metal piping of heavy water nuclear power plants.

A portable laser ablation sampling device was developed for in situ measurements of materials outside of the laboratory. Glaus et $a .^{52}$ reported that the particles generated by the laser ablation sampling were trapped on membrane filters and subsequently analysed using LA-ICP-MS in the laboratory. This provided an elemental fingerprint of the material. This sampling method was tested on glass and gold reference materials. Accuracies of better than $20 \%$ were observed for most elements and typical limits of detection were in the range of 0.01-1 $\mu \mathrm{g} \mathrm{g}^{-1}$.

A review of available copper alloy reference materials that could be used for calibration of LA-ICP-MS for the analyses of ancient copper or bronze artefacts was undertaken by Walaszek et al. ${ }^{53}$ The authors cited 66 references and metallurgical data were presented on the homogeneity and spatial element distribution in these materials. A number of reference materials were examined by optical microscopy and SEM-EDXRF. The effect of inhomogeneities on different ablation and calibration strategies was evaluated and used to define optimum analytical conditions.

A review of the application of XRF to investigations of cultural relics and archaeological finds was written by Musilek et al. ${ }^{54}$ The authors cited 111 references and discussed the advantages and disadvantages of XRF for the analysis of a wide variety of materials (including metals) to assist in determining the origin and production technologies of these materials. An investigation into performance of three different detectors of portable XRF spectrometers was conducted by Guerra et al. ${ }^{55}$ Different reference materials (including brass cuttings NBS-1105) were used to assess how the each detector performed. Limits of detection were estimated for elements present in the reference materials and a coin and two other materials were used as case studies to further assess these detectors.

Flame atomic absorption spectrometry continues to be a useful tool for simple analyses as demonstrated by Dessuy et al. ${ }^{56}$ who determined $S b$ and $P b$ in pewter alloys with a high resolution continuum source FAAS. Samples were dissolved in hydrochloric acid and hydrogen peroxide with a cold finger system to minimize analyte loss. The main resonance line for $\mathrm{Pb}$ at $217.001 \mathrm{~nm}$ and a secondary line for Sb at $212.739 \mathrm{~nm}$ were used and limits of detection were estimated to be 0.02 and $5.7 \mathrm{mg} \mathrm{L}^{-1}$ for $\mathrm{Pb}$ and $\mathrm{Sb}$, respectively. Accuracy was assessed by recovery studies and comparison to results obtained using ICP-OES. Use of $t$-tests demonstrated that there were no significant differences at the $95 \%$ confidence interval between the data observed using the two techniques.

\section{Industrial chemicals}

\subsection{Fuels and lubricants}

This has again been a popular area of research with numerous papers being published that have described methodology for 
the analysis of such materials. Compared with previous years' updates, there has been a sharp decline in the number of papers using continuum source AAS as a means of detection.

2.1.1. Petroleum products-gasoline, diesel, gasohol and exhaust particulates. Several different techniques have been used for the analysis of these materials. Two applications have utilized LIBS to investigate the processes occurring within a flame or an engine. A paper by Zhang et al. ${ }^{57}$ described the use of LIBS to quantify local equivalence ratios of kerosene-air mixtures at elevated pressure in a high temperature test cell that simulates supersonic combustion. A Nd:YAG laser operating at $532 \mathrm{~nm}$ and an energy of $120 \mathrm{~mJ}$ was used to excite the materials and the $\mathrm{H} / \mathrm{O}$ and $\mathrm{H} / \mathrm{N}$ emission signals and equivalence ratio were measured at different gas pressures. In addition, the full width at half maximum (FWHM) of the H-alpha line was also measured at different pressures. It increased in proportion with the pressure. The instrumentation used enabled the temperature to be monitored from room temperature to $350{ }^{\circ} \mathrm{C}$ and the pressure from 1-4 atmospheres. The detection system comprised collection optics, a fibre optic, a low resolution spectrometer $(0.125 \mathrm{~nm}$ resolution at $500 \mathrm{~nm})$ that covered the wavelength region of 200-850 $\mathrm{nm}$ and an intensified CCD camera. The system developed was used successfully to obtain the data required online. A second paper to utilize LIBS was prepared by Buschbeck et al. ${ }^{58}$ who determined local lambda values (the normalized air-fuel mass ratio) in a single cylinder direct injection engine and also the ignition location lambda. A $\mathrm{Nd}$ :YAG laser operating at $1064 \mathrm{~nm}$ in the nanosecond region yielded a laser spot about $10 \mu \mathrm{m}$ in diameter. Initially, the detection system was relatively simple with a cold mirror, bandpass filters and two photomultiplier tubes being used to detect light from $\mathrm{H}$ at $656.2 \mathrm{~nm}$ and from $\mathrm{O}$ at $777 \mathrm{~nm}$. However, this was later replaced with a Czerny-Turner monochromator and an intensified CCD camera. The LIBS data collected enabled variations of ignition location lambda for different fuel injection strategies as well as correlations between variations ignition location lambda and the combustion dynamics to be calculated. Insufficient lean lambda values were the source of misfires in lean combustions. The study was extended to use both LIBS and LIF to obtain additional information on the twodimensional lambda distribution. The instrumental setup was similar to that for the LIBS experiments, but a Nd:YAG laser operating at $266 \mathrm{~nm}$ was used to excite molecules into fluorescing at their most sensitive region, i.e. $<320 \mathrm{~nm}$. The setup and operation were discussed at length in the paper.

Several other techniques have been used to analyse these materials and these will be discussed individually. Cunha et al. ${ }^{\mathbf{5 9}}$ determined $\mathrm{Cu}, \mathrm{Cr}$ and $\mathrm{Pb}$ in gasoline and naphtha using a piston-propelled flow batch analyser and ETAAS detection. The flow batch analyser was used to prepare micro-emulsions of the materials in a surfactant (Triton X-100), water and an alcohol (propanol, heptanol, cyclohexanol, tert-butanol and isopropanol were all tested). In the study of gasoline samples, the Triton X-100 was diluted with tert-butanol whereas for the naphtha, propanol was used to dilute the emulsifying agent. Once prepared, the emulsions were delivered by the flow batch analyser to an autosampler cup of the ETAAS instrument and the analytes determined. The design and programme of the flow batch analyser as well as the temperature programme of the ETAAS instrument were presented. Since very high concentrations of Triton X-100 were used (between 25 and 75\%), the solutions were very viscous. The authors, therefore, undertook a careful rheological evaluation to ensure that the individual components could be transported efficiently. Data obtained using the automated method of micro-emulsion formation were compared with those obtained manually and, according to a paired $t$-test, were in agreement. Recoveries were quantitative, with $\mathrm{Pb}$ in naphtha being recovered at $100.2 \pm 3.4 \%$ and $\mathrm{Cu}$ and $\mathrm{Cr}$ in gasoline at $100.7 \pm 4.6 \%$.

A second paper to use ETAAS as a means of detection was prepared by Becker et al. ${ }^{60}$ who determined As in acid digests of gasoline samples using a HG-ETAAS method utilizing iridium as a permanent modifier. A full two level factorial design with centre point was used to optimize the system. The sample volume, the pre-reduction conditions, the temperature programme and the mass of iridium used $(150 \mu \mathrm{g})$ were kept constant whilst the effects of reducing agent concentration (negative effect), acid concentration (negative effect) and trapping temperature (positive effect) were determined. Optimal parameters for arsine generation were: $2.7 \mathrm{~mol} \mathrm{~L}^{-1}$ hydrochloric acid, $1.6 \%$ $(\mathrm{w} / \mathrm{v})$ sodium tetrahydroborate, a collection temperature of $250{ }^{\circ} \mathrm{C}$ for a time of $30 \mathrm{~s}$. An impressive LOD of $6.4 \mathrm{ng} \mathrm{\textrm {L } ^ { - 1 }}$ was obtained which corresponded to an absolute amount of $24 \mathrm{pg}$. Two different acid digestion systems (a digester block with a cold finger using sulfuric acid and a microwave oven using nitric acid) were compared and the results obtained also compared with those obtained using a method in which the samples were introduced to ETAAS as a micro-emulsion and determined directly. In general, the results were comparable, with those obtained using the microwave oven being in particularly good agreement with those obtained using the reference method.

Universal calibration for metal determination in fuels and biofuels using segmented flow injection into a heated spray chamber of an ICP-OES instrument was described by Sanchez et $a{ }^{61}$ A single pass spray chamber equipped with a brass hollow cylinder for heating was used to introduce the sample. The sample itself could be gasoline, superethanol, diesel or biodiesel. All sample types were diluted $1: 1$ with xylene except for superethanol which required a $1: 3$ dilution to prevent plasma extinction. An aliquot $(<5 \mu \mathrm{L})$ of the diluted sample was introduced into the system for analysis. Optimization of the heated spray chamber found that sensitivity increased with increasing temperature. The heated spray chamber had the added benefit of decreasing both memory and matrix effects. The latter were virtually eliminated at $350{ }^{\circ} \mathrm{C}$ because, irrespective of type, the sample was completely vaporized into the plasma. Consequently, LOD of better than $7 \mu \mathrm{g} \mathrm{L}^{-1}$ were obtained for analytes such as $\mathrm{Mn}, \mathrm{Si}$ and V. Calibration was achieved using different volumes of a single xylene-based solution as a universal standard. Recoveries were in the range 95-106\%.

Vorapalawut et al. ${ }^{62}$ developed a method by which Fe, Ni, S and $\mathrm{V}$ distribution in various fractions of crude oils could be 
characterized using a combination of thin layer chromatography (TLC) and LA-ICP-MS. Crude oils and their fractions (saturated, aromatic, resin and asphaltene) were placed on an activated silica gel plate and separated using a selection of different solvents. Each band of material was then placed on a microscope slide and analysed using LA-SF-ICP-MS. Optimization of the LA process indicated that a scan process of $80 \mu \mathrm{m} \mathrm{s}^{-1}$, a laser energy of $50 \%(0.45 \mathrm{~mJ})$ and a spot size of $200 \mu \mathrm{m}$ were optimal. The LOD for the analytes Ni and V which are present at trace level were 18 and $23 \mathrm{ng} \mathrm{g}^{-1}$ respectively. A sample could be characterized within 10 minutes.

2.1.2. Fuel-coal, peat and other solid fuels. A review entitled "analytical methods for mercury analysis in coal and coal combustion by-products" containing 115 references was prepared by Lopez-Anton et al. $^{63}$ The review focussed on the specific characteristics of each of the sample types and the problems associated with their analysis. The methods used to achieve the analyses (both those under development and standard methods) were described, along with their associated advantages and disadvantages. The focus was mainly on the quality of the data produced and the uncertainties.

Recent progress in the use of LIBS for monitoring coal quality and unburned carbon in fly ash was summarized by Zhang et al. ${ }^{\mathbf{6 4}}$ Although described as a review, the references cited within the paper indicate that it is much more of a discussion of LIBS for assorted sample types. However, the recent progress in the area of interest in the authors' own laboratories were also summarized. A software-controlled LIBS system comprising a selfcleaning device for online coal quality monitoring in power plants was developed and discussed. The automated sampling device was capable of elemental analysis (where the analytes included $\mathrm{Al}, \mathrm{C}, \mathrm{Ca}, \mathrm{Fe}, \mathrm{H}, \mathrm{Mg}, \mathrm{S}, \mathrm{Si}$, Ti and organic O), as well as proximate analysis. This was made possible through the use of optimal data processing methods that were capable of correcting for both spectral interferences and matrix effects.

There have been several other papers published that have utilized LIBS to determine analytes in such materials. Included in this number is a paper by Yuan et al. ${ }^{65}$ who determined $\mathrm{C}$ in anthracite powders. The quantitative determination of $\mathrm{C}$ in anthracite powders has historically been difficult using LIBS because of the high measurement uncertainty. These authors bound the powder together using sodium silicate and then used the $\mathrm{Si}$ as an internal standard. This yielded data with better measurement precision and accuracy. A total of 15 anthracites were used for calibration and a further seven for validation. Using the optimal amount of binder, the precision was $12.1 \%$ RSD and the root mean square error of prediction was $6.25 \%$. The same research group also determined $\mathrm{C}, \mathrm{H}$ and $\mathrm{N}$ in 24 bituminous coal samples using LIBS. ${ }^{66}$ Argon and helium were compared as an ambient gas to prevent the ingress of nitrogen from the atmosphere and to enhance the emission signals. Partial least squares modelling was used to compare the different ambient gases. In general, the argon provided better precision through a decrease in signal fluctuation and a greater enhancement in sensitivity. The root mean square error of prediction for $\mathrm{C}$ concentration decreased from $4.25 \%$ in air to $3.49 \%$ in argon, whilst the average relative error decreased from
4.96 to $2.98 \%$. Similar improvements were observed during $\mathrm{H}$ determination. The determination of $\mathrm{N}$ proved impossible. Although a distinct signal was observed using air as an ambient gas, it disappeared when argon was used which indicated that the signal arose from the $\mathrm{N}$ in the air rather than from the sample.

The final LIBS paper in this section was provided by Dong et al. ${ }^{67}$ who also compared air, argon and helium at atmospheric pressure as ambient gas for the determination of atomic and molecular $C$ in coal. These authors used a Q-switched Nd:YAG laser operating at $266 \mathrm{~nm}$ and with a power of $14 \mathrm{~mJ}$ and time resolved analysis to monitor the signal arising from C, $C_{2}$ and $\mathrm{CN}$. Both the optical setup and the preparation methodology for the coal samples were described in the paper. Briefly, the powdered coal of $100 \mu \mathrm{m}$ grain size was pressed using 25 tons into a pellet $5 \mathrm{~mm}$ thick and $31 \mathrm{~mm}$ in diameter. The laser was fired a total of 50 times per site and at four sites per pellet to ensure any statistical evaluation was valid. These authors also used atomic Fe emission lines to construct Boltzmann plots to determine plasma excitation temperature. Computer simulations of $C_{2}$ spectra were used to deduce molecular rotational temperature and the electron density and total atomic and molecular number density were reported to deduce emission differences between atomic and molecular C. The total molecular number and the reactions between plasma species and the ambient gas were the main factors affecting molecular $\mathrm{C}$ emission. The influence of laser energy on the rotational temperature in an air atmosphere was also studied and demonstrated that $C_{2}$ band emission could be utilized to correct for plasma fluctuations.

Other applications of coal analysis have also been reported. In one, by Guo et al. ${ }^{68}$ the characterization of $\mathrm{Hg}$ in coals was undertaken using a temperature-programmed decomposition with $\mathrm{Hg}$ detection using AFS. Acid leaching followed by $\mathrm{Hg}$ detection was also used to assist in the characterization. In general, the content of organically-bound $\mathrm{Hg}$ was in the range $16-36 \%$ and was released in the temperature region of $220-600{ }^{\circ} \mathrm{C}$. Silicate bound $\mathrm{Hg}$ constituted $10-26 \%$ of $\mathrm{Hg}$ and was released at temperatures greater than $600{ }^{\circ} \mathrm{C}$. Pyrite-bound $\mathrm{Hg}$ was typically $5-44 \%$ of total $\mathrm{Hg}$ and was released between 250 and $630{ }^{\circ} \mathrm{C}$. Finally, hydrochloric acid soluble $\mathrm{Hg}$ ranged between 10 and $34 \%$ and was released at lower temperatures. Six Chinese coals were used during the study.

Two papers have used ICP-OES as a means of detection after using different extraction techniques. Although the atomic spectrometry in both papers is not terribly novel, they do present viable methods of sample preparation and have therefore been included in the review. In one of these papers, Low and Zhang, ${ }^{69}$ optimized a microwave extraction technique from coal and coal fly ash prior to the ICP-OES determination of six major elements ( $\mathrm{Al}, \mathrm{Ca}, \mathrm{Fe}, \mathrm{K}, \mathrm{Mg}$ and $\mathrm{Na}$ ) and 12 trace elements. Seven reference samples including two coal certified materials (the South African Bureau of Standards material SARM 19 and NIST SRM 1632c) and three coal fly ash reference materials (SRM 1633c, SRM 2690 and BCR 38) were used during the optimization process. The recoveries of these elements in the different samples were tested as a function of the amount of 
hydrofluoric acid used (over the range 0-2 $\mathrm{mL}$ ), microwave power $(900 \mathrm{~W}$ or $1200 \mathrm{~W}$ ) and sample mass $(0.05$ or $0.1 \mathrm{~g})$. The amount of nitric acid $(5 \mathrm{~mL})$ and hydrogen peroxide $(2 \mathrm{~mL})$ remained constant throughout. The use of hydrofluoric acid was not necessary for the majority of elements in coal samples, the exceptions being $\mathrm{K}, \mathrm{Pb}$ and $\mathrm{V}$. In particular, the $\mathrm{Pb}$ extraction was noted as being highly sample specific and hence unpredictable. Conversely, the coal fly ash samples required the use of 0.1-0.2 mL hydrofluoric acid to obtain complete recovery. Even with the use of the hydrofluoric acid, the recovery of Al was never quantitative.

The other paper was prepared by Mujuru et al. ${ }^{70}$ who used $N, N$-dimethylformamide (DMF) and ultrasound to assist the extraction of five trace elements from slurries of coal samples. Coal $(2 \mathrm{~g})$ was placed in a plastic cup containing agate grinding elements and then DMF $(5 \mathrm{~mL})$ was added. After sealing the cup, it was placed in a micronizing mill for a period of $30 \mathrm{~min}$. The coal slurry was placed in a $200 \mathrm{~mL}$ capacity volumetric flask, Triton X-100 was added as a dispersant and sufficient nitric acid added to give a final concentration of $2 \%$. The flasks were placed in a sonic bath for 15 minutes to ensure good dispersion of particles. The slurry was then analysed using ICP-OES. Results obtained were compared with those obtained using a dry slurry technique. With this technique $2 \mathrm{~g}$ of coal were ground in the absence of DMF and then a $0.5 \mathrm{~g}$ aliquot placed in a $25 \mathrm{~mL}$ volumetric flask, and the Triton X-100 and nitric acid added. These slurries were also sonicated prior to analysis. To determine the amount of analyte extracted from the particles, aliquots of the slurries were passed through a $0.45 \mu \mathrm{m}$ filter. The concentrations in the supernatant and the slurry could, therefore, be compared. Although there was some extraction in the absence of DMF, greater extraction was found in its presence. The proportions of the analytes extracted were very variable, with that for $\mathrm{V}$ being the lowest $(17 \%)$ and that for $\mathrm{Pb}$ being the highest (100\%). Studies using SEM indicated that partial dissolution of the slurry particles occurred. The procedure developed was applied to the analysis of three bituminous coal samples from different seams from South Africa.

Lusilao-Makiese et al. ${ }^{71}$ determined total $\mathrm{Hg}$ in coals using ICP-MS but also developed a method of $\mathrm{Hg}$ speciation that involved a four stage sequential leaching process followed by determination using ID-GC-ICP-MS. Numerous $\mathrm{Hg}$ species were identified in the coals including $\mathrm{Hg}^{0}, \mathrm{Hg}^{2+}$ and $\mathrm{CH}_{3} \mathrm{Hg}^{+}$. Other species, e.g., ethylmercury, were also identified in some samples. Organic-bound $\mathrm{Hg}$ was typically $37-40 \%$, sulfidebound $\mathrm{Hg}$ constituted approximately $37-39 \%$ and the use of hydrochloric acid enabled $16 \%$ of the $\mathrm{Hg}$ to be extracted.

2.1.3. Oils-crude oil, lubricants. Sample preparation methods for subsequent determination of metals and non-metals in crude oils were reviewed (with 207 references) by Mello et al. ${ }^{72}$ The review combined text and easy reference tables to provide the necessary information and covered topics as diverse as acid decomposition methods (including the use of microwave technology), combustion bombs, simple dilution in organic solvents (the dilute and shoot approach), emulsification, and methods that can be used for speciation analysis. Also discussed were the direct analysis methods, e.g., ETAAS, XRF, etc., which require little or no sample preparation. A useful addition to the review was a section that discussed available reference materials. The authors point out that unfortunately many of the reference materials are either diesels, petrol or wear oils. Many of them have certified values for $\mathrm{S}$ and $\mathrm{a}$ few for $\mathrm{Ni}$ and $\mathrm{V}$. However, there are few materials that are certified for a suite of analytes.

Acid decomposition protocols are still popular for this sample type because ICP instrumentation "prefers" aqueous phase solvents to be aspirated. However, the conditions required for complete dissolution are usually very harsh. Ortega et al. ${ }^{73}$ compared several different acid dissolution protocols including three separate microwave digestion procedures (that used different temperatures and pressures), a microwave combustion method and a high temperature ashing method. The latter comprised putting crude oil (500 mg) in a quartz container, adding nitric acid and hydrogen peroxide and then heating to $320{ }^{\circ} \mathrm{C}$ in an autoclave. The paper gave the full experimental conditions for each of the protocols. As well as giving the relative recoveries obtained from the analysis of the certified material NIST SRM 1634c, the authors also commented on the quality of the blank solutions and on the time required per digestion. Although the microwave induced combustion was the most rapid, it did suffer from poor quality blanks. It also used a far higher temperature $\left(1400{ }^{\circ} \mathrm{C}\right)$ than any of the other protocols. In general, recovery using the different protocols ranged from 80 to $120 \%$, with one of the acid digestion procedures giving a consistently high recovery rate of between 91 and $105 \%$ for all of the analytes determined. Other protocols, e.g., the microwave induced combustion, were far more variable, with recoveries ranging from 84.4 to $119.9 \%$ depending on the analyte.

Another paper to have compared different sample decomposition methods was prepared by Pontes et al. ${ }^{74}$ These authors compared three methodologies for the preparation of crude oil and related products prior to the determination of total $\mathrm{Hg}$ using $C V-A A S$. These three methodologies were: a closed-pressurized vessel, a cold finger digest collection system placed in an aqua regia heated digestion apparatus, and a pyrolysis system. The loss of sample through evaporation was determined for each of the methods, with the pyrolysis system suffering the worst loss rates, largely because it used the smallest mass of material ( $\sim 25 \mathrm{mg}$, compared with $0.25 \mathrm{~g}$ and $0.5 \mathrm{~g}$ for the high pressure asher and cold finger systems respectively). Methods designed to minimize these problems were investigated. Since the cold finger system was large and heavy, the sample could not be weighed directly into it. The authors, therefore, used gelatinous capsules, similar to those used in the pharmaceutical industry to enclose drugs, to contain the samples. Sample was simply weighed into one half of the capsule and was then enclosed using the other half, with the whole capsule being transferred into the digestion system. Despite the development of this weighing protocol, the authors stated that this method had the highest blank levels, the highest LOQ and the longest digestion time of all the methods tested. The pyrolysis system used only $\sim 25 \mathrm{mg}$ of sample, since higher mass led to interference effects through the production of smoke, yielding recoveries of $\mathrm{Hg}$ between 63 and $180 \%$. Placement of the sample on alumina 
powder within the pyrolysis chamber decreased evaporation losses significantly (by between 38 and 61\%) and had the added bonus of facilitating the cleaning of the chamber after use. The LOD obtained depended on the method used for sample preparation but all were impressive, with values of $1.3,8.6$ and $3.3 \mathrm{ng}$ $\mathrm{g}^{-1}$ being achieved for the high pressure ashing, cold finger and pyrolysis methods respectively. Precision was better than $14 \%$ RSD for all procedures.

A different approach to acid digestion was presented by Cinosi et al. ${ }^{75}$ who described atmospheric pressure, vapour phase decomposition of high boiling petrochemical fractions and distillation bottoms. Sample was attacked by nitric acid vapours that had been heated to $100{ }^{\circ} \mathrm{C}$ and the digest analysed using TXRF. The LOD obtained were in the range of $10-100 \mu \mathrm{g} \mathrm{kg}^{-1}$ for $\mathrm{As}, \mathrm{Bi}, \mathrm{Co}, \mathrm{Cr}, \mathrm{Cu}, \mathrm{Fe}, \mathrm{Mn}, \mathrm{Ni}, \mathrm{Pb}, \mathrm{Sr}$ and $\mathrm{Zn}$ whereas they were higher (0.3-3 $\mathrm{mg} \mathrm{kg}^{-1}$ ) for $\mathrm{Ba}, \mathrm{Ca}, \mathrm{K}, \mathrm{P}, \mathrm{Rh}$, Ti and $\mathrm{V}$. The preparation procedure was rapid, lasting less than 30 minutes. The results were compared with those obtained using the standard ASTM D5708 method B, that requires the use of a sulfuric acid wet ash followed by a period in a muffle furnace to remove the carbonaceous residue and the uptake of the remaining residue using nitric acid prior to ICP-OES determination. The digestion protocol developed and reported in this paper was significantly faster, since the standard method takes typically a whole day. Results obtained using the two protocols were in reasonable agreement, with a regression coefficient of 0.92 being achieved. Precision values were also similar.

The determination of As and Se in crude oil using a simple dilution in xylene and analysis using ICP-MS was reported by de Albuquerque et al. ${ }^{76}$ The dynamic reaction cell was not required for the determination of As, since there was no visible interference on $\mathrm{m} / \mathrm{z} 75$. However, the introduction of methane into the cell at a rate of $0.5 \mathrm{~mL} \mathrm{~min}^{-1}$ was required for the determination of Se at $m / z$ 80. Results obtained using this simple "dilute and shoot" method were compared with those obtained using a nitric acid decomposition of the material in a hotblock followed by reduction using $6 \mathrm{M}$ hydrochloric acid and analyte determination using HG-ICP-MS. The results were in good agreement. The reference method provided significantly lower LOD (0.002 and $0.0414 \mu \mathrm{g} \mathrm{kg}{ }^{-1}$ for As and Se, respectively) compared with the proposed method $\left(0.04\right.$ and $0.1 \mu \mathrm{g} \mathrm{kg}^{-1}$ for As and Se). However, it was much slower. Further method validation for the proposed method was achieved using certified reference materials. Despite its short-comings, the proposed method was deemed fit for purpose.

Two papers by Ortega et al. ${ }^{77,78}$ discussed the determination of $\mathrm{Pb}$ isotope ratios in crude oils and related materials. In one ${ }^{78}$ oil $(0.5 \mathrm{~g})$ was digested using hydrogen peroxide and nitric acid in a high pressure asher. Half of the digest was used to determine total $\mathrm{Pb}$ using ICP-MS and the other half underwent $\mathrm{Pb}$ isolation and purification using AG1-X8 resin prior to $\mathrm{Pb}$ ratios being measured using MC-ICP-MS. The protocol for this was described in detail. Poor recovery from the resin led the authors to optimize the amount of resin used, and at least $0.5 \mathrm{~mL}$ was optimal. Several mass bias correction procedures were evaluated and a combination of thallium normalization and standard bracketing was optimal. Precisions were 0.0039, 0.0014 and $0.0012 \%$ and accuracies (in parts per thousand) were 0.001 , 0.001 and 0.004 for ${ }^{208} \mathrm{~Pb} /{ }^{204} \mathrm{~Pb},{ }^{207} \mathrm{~Pb} /{ }^{204}$ and ${ }^{206} \mathrm{~Pb} /{ }^{204} \mathrm{~Pb}$, respectively for the analysis of NIST SRM 981. The other paper ${ }^{77}$ also used an acid digestion but then derivatized the $\mathrm{Pb}$ using tetraethylborate forming tetraethyl lead. This was extracted into isooctane and then injected into a GC-MC-ICP-MS system. Again, thallium was used as a means of mass bias correction, being introduced as a liquid sample aspirated simultaneously to the eluent from the GC. Results obtained using the method were compared with those obtained using conventional continuous sample introduction.

A simple emulsion protocol for the determination of $\mathrm{Ba}, \mathrm{Ca}$, $\mathrm{Mg}$ and $\mathrm{Sr}$ in crude oils using FAAS (for $\mathrm{Ca}, \mathrm{Mg}$ and $\mathrm{Sr}$ ) and ETAAS (for Ba) was described by Pessoa et al. ${ }^{79}$ The amount of sample required was dependent on the analyte, but was approximately $0.1 \mathrm{~g}, 0.25 \mathrm{~g}$ and $1 \mathrm{~g}$ for $\mathrm{Mg}$, Ca and $\mathrm{Sr}$ respectively. Sample was diluted with $1 \mathrm{~mL}$ of toluene, sonicated using a sonic bath for five min and then Triton X-100 (100 mg) and potassium chloride $(0.5 \mathrm{~mL}$ of $2 \%)$ added. Finally, the sample was diluted to $10 \mathrm{~mL}$ using $0.2 \% \mathrm{v} / \mathrm{v}$ nitric acid, shaken manually and replaced in the sonic bath for a further five minutes. Calibration standards were prepared in a similar way but using base oil and aqueous standards. The emulsions were stable for at least five days. Recoveries ranged from 96 to 104\% and the results obtained from the analysis of the reference material NIST SRM 1085b were in good agreement with certified values.

A pressurized liquid extraction method for the determination of $\mathrm{Al}, \mathrm{Cu}, \mathrm{Mg}, \mathrm{Pb}, \mathrm{Sn}$ and $\mathrm{Ti}$ in lubricating oils was developed by Carballo-Paradelo et al. ${ }^{80}$ who used dilute $\mathrm{HCl}$ as extractant. A very specific order of loading the extraction cell was required and this was given in the paper. A Plackett-Burman experimental design was used to study the variables: $\mathrm{pH}$ of the aqueous extractant, mass of dispersing agent, mass of cleaner agent, temperature and time of the extraction cycle and the number of cycles. The most important of these variables was the $\mathrm{pH}$, which had to be set at 0.5 using concentrated hydrochloric acid. Using ICP-MS as a means of detection, the recoveries obtained when a reference material (NIST SRM 1084a) was analysed ranged from 80 to $118 \%$, although $\mathrm{Ag}$ was much poorer (typically $\sim 20 \%$ ). The authors stated that the method had several advantages over other procedures because it dealt solely with aqueous extracting solutions, which means that calibration standards could also be aqueous-based. In addition, fewer interferences were observed because organic solvents were not introduced to the plasma.

A simple, direct method of determining As and Cd in crude oils was described by de Jesus et al. ${ }^{\mathbf{8 1}}$ Sample was weighed directly onto solid sampling platforms and then analysed using ETAAS. A matrix modifier of $0.1 \%$ palladium, $0.06 \%$ magnesium and $0.06 \%$ Triton X-100 was suitable for both analytes and calibration was against aqueous calibrants. Under optimal conditions (ash and atomize curves were plotted for both aqueous standards and for the crude oil samples), detection limits were 5.1 and $0.2 \mu \mathrm{g} \mathrm{kg}^{-1}$ for As and Cd respectively. As usual with direct solid sampling, precision was not particularly good, but was described as being better than 20\% RSD. Method validation was 
achieved in two ways. For the As determination, the reference material NIST SRM $1634 \mathrm{c}$ was used. However, no suitable material was available for the $\mathrm{Cd}$ and so an alternative method was employed. This involved the open vessel digestion of the sample using a cold finger to achieve reflux. Using the paired $t$-test, the results obtained using this method were compared with those obtained using the solid sampling protocol and were not significantly different.

Pontes et $a l^{82}$ determined $\mathrm{Hg}$ in crude oils and related products by developing an online system that utilized a gold trap to preconcentrate the $\mathrm{Hg}$ vapour prior to determination using $\mathrm{CV}$ $A A S$. The preparation of the gold trap was described in detail as was the FI manifold that utilized numerous valves to inject sample, direct argon flow etc. The $\mathrm{Hg}$ was released from the samples using tin(II) chloride and the $\mathrm{Hg}$ was then trapped on a gold amalgamation trap. This was then heated to release the $\mathrm{Hg}$ so that it could be detected using a Zeeman effect CV-AAS instrument. The use of the Zeeman effect background correction system was described as being beneficial because it helped overcome interference effects caused by any organic vapours released from the trap. Numerous parameters were optimized including the amalgamation temperature, amalgamation time and the argon purge gas flow rate. Trapping at $120^{\circ} \mathrm{C}$ provided a sharper peak once the $\mathrm{Hg}$ was released than collection at room temperature, which provided a distorted, broad peak and led to over estimates of the analyte concentration (135\% recovery). The release temperature was a compromise between the high temperatures required to avoid non-Gaussian shaped peaks and durability of the heating element of the trap. Above $600{ }^{\circ} \mathrm{C}$, the nickel-chromium alloy heating element became brittle and snapped. A maximum of $600{ }^{\circ} \mathrm{C}$ was therefore employed. An amalgamation time of three minutes was sufficient to obtain complete recovery from $2.5 \mathrm{~mL}$ of crude oil spiked with $1 \mu \mathrm{g} \mathrm{L} \mathrm{L}^{-1}$ $\mathrm{Hg}$. The LOD obtained was $0.06 \mu \mathrm{g} \mathrm{\textrm {L } ^ { - 1 }}$ and precision was better than $10 \%$ RSD. Recoveries of between 85 and $116 \%$ were achieved for an assortment of sample types including crude oils, diesel and gas condensates.

The use of xerogels for elemental analysis of crude oils using LAICP-MS was described by Martinez et al. ${ }^{83}$ Crude oil $(0.5000 \mathrm{~g})$ was dissolved in dichloromethane and then added to zirconium n-propoxide. This mixture was then added to ethanol, water, nitric acid and Tergitol to form the xerogel. This was dried, mixed with boric acid and pelletized prior to LA-ICP-MS analysis using a Nd:YAG laser and a sector field ICP-MS instrument. An internal standard of indium was used to correct for variation in the amount of sample ablated. Calibration was achieved using aqueous standards encapsulated in a xerogel in a similar fashion to the oils. Results for Ni, S and V obtained using this method were compared with those obtained using either direct introduction of the organic sample (in the case of $\mathrm{Ni}$ and $\mathrm{V}$ ) or by a digestion (for $\mathrm{S}$ ) and were similar. Excellent sensitivity and good precision were obtained.

2.1.4. Alternative fuels. Over the last few years, there have been a growing number of applications of the analysis of alternative fuels and this trend has continued during this review period. A particularly high proportion of the papers in this section originate from South America in general and Brazil in particular. Presumably this is because of the amount of alternative fuels used in these countries.

The development of suitable certified reference materials is a requisite for method validation for any sample type. During this review period, Zhu et al. ${ }^{84}$ reported the development of a material certified for $\mathrm{Cu}$ in bio-ethanol (NMIJ CRM 8301-a). The material was made from rice fermentation and was mixed with copper acetate and acetic acid. Samples were diluted by a factor of 10 using $1 \mathrm{M}$ nitric acid and then analysed using a number of techniques including gravimetric standard addition ICP-MS, ID-ICP-MS and ETAAS. The combined uncertainty (3.79\% relative value) of the certified value was calculated as a combination of the uncertainties of homogeneity (3.53\%), analytical method $(0.64 \%)$ method to method variance $(1.16 \%)$ and calibration standards $(0.35 \%)$. The material was certified as having a $\mathrm{Cu}$ content of $53.7 \pm 4.1 \mathrm{ng} \mathrm{g}^{-1}$.

Three papers have reported a simple "dilute and shoot" approach to the determination of analytes in biodiesel. Amais et $a l .{ }^{85}$ diluted the material 10 -fold in ethanol prior to the determination of Si using a nitrogen MIP-OES, with sample introduction using a flow blurring nebulizer and a cyclonic, double pass spray chamber. The method was compared with two others. In one, a micro-emulsion was formed by mixing sample $(1 \mathrm{~mL})$ with Triton X-100 $(0.5 \mathrm{~mL})$ and $20 \%$ nitric acid $(0.5 \mathrm{~mL})$ followed by dilution with $\mathrm{n}$-propanol to $10 \mathrm{~mL}$. The second comparative method used a microwave assisted acid digestion approach in which samples $(0.2 \mathrm{~g})$ were mixed with $50 \%$ nitric acid $(5 \mathrm{~mL})$ and hydrogen peroxide $(3 \mathrm{~mL})$. Detection limits ranged from 5 to $20 \mu \mathrm{g} \mathrm{L}^{-1}$ and precision was better than $2 \%$ RSD for all methodologies. The dilute and shoot approach was faster and far more simple than the other protocols and seemed not to have any drawbacks. It even enabled calibration using inorganic standards. Another paper to use a dilute and shoot approach was prepared by Caldas et al. ${ }^{86}$ who applied a four variable Doehlert design for the multivariate optimization of $\mathrm{Cu}$ determination using ETAAS in petroleum-derived insulating oils. The oil was diluted 1:1 with hexane and then injection $(20 \mu \mathrm{L})$ into the ETAAS instrument. The Doehlert design optimized the drying, ash and atomization temperatures, the ash time and the heating ramp during the drying step. Under optimal conditions, the LOD achieved was $1.6 \mu \mathrm{g} \mathrm{L}^{-1}$ and the LOQ was $5.3 \mu \mathrm{g} \mathrm{L}^{-1}$. Even though optimal conditions were used, the method of standard additions was required since interferences were not removed completely. This would, of course, slow the rate of analysis considerably.

The third paper to use the dilution approach was reported by de Souza $e t ~ a l .{ }^{87}$ who diluted an assortment of oil matrices by a factor of 10 using xylene and then analysed the dilute samples using ICP-MS employing a reaction cell gas of methane. Calibration was achieved using a commercial organic-based, multielemental standard diluted in xylene. A specialist organic material introduction kit comprising a micro-nebulizer, a cryogenic chamber kept at $5{ }^{\circ} \mathrm{C}$ and a demountable torch with an injector with a bore of only $0.85 \mathrm{~nm}$ was used. A small flow of oxygen $\left(0.1 \mathrm{~L} \mathrm{~min}^{-1}\right)$ was introduced between the outlet of the spray chamber and the torch to minimize the amount of carbon being deposited on the interface and ion lens system of the 
instrument. The chemometric optimization technique of Central Composite Design was used to optimize ICP operating parameters such as power and the nebulizer and auxiliary gas flows ensuring that doubly charged ion $\left(\mathrm{Ba}^{2+}\right)$ and oxide-related $\left(\mathrm{LaO}^{+}\right)$interferences were minimized. The optimal conditions were: nebulizer gas flow rate of $0.42 \mathrm{~L} \mathrm{~min}^{-1}$, auxiliary gas flow rate of $1.1 \mathrm{~L} \mathrm{~min}{ }^{-1}$ and a power of $1300 \mathrm{~W}$. Addition of methane to the reaction cell was employed to overcome any interferences during the determination of ${ }^{40} \mathrm{Ca},{ }^{52} \mathrm{Cr},{ }^{56} \mathrm{Fe}$ and ${ }^{24} \mathrm{Mg}$. The amount of methane required was not the same for all analytes,

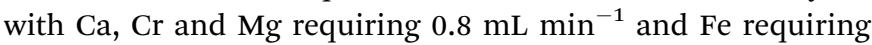
only $0.1 \mathrm{~mL} \mathrm{~min}^{-1}$. Use of a methane flow rate higher than $0.1 \mathrm{~mL} \mathrm{~min}^{-1}$ actually increased interference effects exerted on the Fe signal. The work was validated using NIST SRM 1034c (residual fuel oil) and SRM 1085b (wear metals in lubricant oil) and was then applied to biodiesel samples that included cotton, sunflower, used frying oil, palm, pequi, soybean, animal fat and castor bean oil. A suite of analytes were determined, with most giving recoveries in the range $90-110 \%$ but with $\mathrm{Mg}$ giving a recovery of $119 \%$. Detection limits ranged between $0.2 \mu \mathrm{g}$ $\mathrm{kg}^{-1}(\mathrm{Cr})$ and $40 \mu \mathrm{kg}^{-1}(\mathrm{Zn})$.

Aranda et al. ${ }^{88}$ developed a method by which $\mathrm{Cd}$ and $\mathrm{Hg}$ were determined in biodiesel using ETAAS with the sample being introduced as an emulsion. Sample was weighed and mixed with $0.25 \mathrm{~mL}$ of nitric acid. This was left for $15 \mathrm{~min}$ and then Triton $\mathrm{X}-100$ and isopropyl alcohol added. After sonication in an ultrasonic bath for 15 minutes, water was used to dilute the emulsion. Aliquots of $10 \mu \mathrm{L}$ were injected for analysis along with a palladium-magnesium matrix modifier. Ash and atomize curves were constructed to obtain optimal instrumental operating conditions. The presence of interferences again necessitated the use of standard additions for the determination of both analytes. The stability of the emulsion was also considered, with a ratio of $4: 1$ sample : nitric acid being optimal. Limits of detection and precision values were $10.2 \mu \mathrm{g} \mathrm{kg}^{-1}$ and $5.5 \%$ for $\mathrm{Hg}$ and $0.3 \mu \mathrm{g} \mathrm{kg}{ }^{-1}$ and $4.4 \%$ for Cd. Results obtained using the emulsification method were compared with those obtained following a microwave assisted acid digestion.

As well as the paper by de Souza $e t$ al. ${ }^{87}$ (discussed above), two other papers have discussed methods of minimizing interferences during ICP-MS analysis of such materials. In one, by Donati et al. ${ }^{89}$ an interference standard and an oxide detection system were employed to determine $\mathrm{P}$ and $\mathrm{S}$ in different oil types. An interference standard is similar in concept to the use of a conventional internal standard, but instead of spiking the sample with indium or another analyte not present at significant quantity in the sample, it relies on a polyatomic interference or an argon species present in the plasma. On this occasion, the ${ }^{30} \mathrm{Si}^{16} \mathrm{O}^{1} \mathrm{H}^{+}$was used in an attempt to maximize accuracy. A univariate approach was taken to optimize the operating conditions, with power, nebulizer flow rate, dwell time and sampling depth all being optimized to give maximum intensities for ${ }^{31} \mathrm{P}^{16} \mathrm{O}^{+}$at $m / z 47$ and for ${ }^{32} \mathrm{~S}^{16} \mathrm{O}^{+}$and ${ }^{32} \mathrm{~S}^{16} \mathrm{O}^{1} \mathrm{H}^{+}$at $\mathrm{m} / \mathrm{z} 48$ and 49 respectively. The procedure developed appeared to depend on the fact that $\mathrm{Ti}$, which would provide several isobaric interferences at the mass to charge ratios being determined, is not a common contaminant of oils. Therefore, although the method appeared to work satisfactorily for the samples analysed, it may be completely inappropriate for many other sample types. The oils were prepared for analysis using a microwave oven assisted acid digestion protocol. Also included in this paper was the use of an interference standard to improve accuracy. The authors claim that it is efficient for most determinations, but inspection of some of the data included in the paper indicates that recovery varied from 62 to $196 \%$. The same research group published a paper that determined several analytes in fuel ethanol. ${ }^{90}$ These authors tested the use of both hydrogen and helium as collision reaction interface gases in an attempt to maximize the efficiency of interference removal during ICP-MS analyses. Sample was diluted 20 -fold and this was sufficient to prevent any carbonaceous build-up on the interface cones. A central composite design approach was used to optimize plasma power, sampling depth and nebulizer gas flow rate. Oxygen addition through the skimmer cone was more efficient in removing polyatomic interferences $\left({ }^{40} \mathrm{Ar}^{12} \mathrm{C}^{+}\right.$, ${ }^{40} \mathrm{Ar}^{16} \mathrm{O}^{+},{ }^{12} \mathrm{C}_{2}^{+}$, and ${ }^{12} \mathrm{C}^{16} \mathrm{O}^{+}$on ${ }^{52} \mathrm{Cr}^{+},{ }^{56} \mathrm{Fe}^{+},{ }^{24} \mathrm{Mg}^{+}$and ${ }^{28} \mathrm{Si}^{+}$ respectively) than addition via the auxiliary gas flow or the introduction of helium. Calibration was achieved using matrix matched, inorganic standards and $\mathrm{Y}$ was used as the internal standard. The protocol developed was applied to the determination of a suite of 16 analytes in fuel ethanol. Recoveries and precision ranged from 78 to $114 \%$ and 0.4 to $2 \%$, respectively. The LOD obtained were better than $0.8 \mu \mathrm{g} \mathrm{L}^{-1}$ for all analytes except for Si, which had a LOD of $14 \mu \mathrm{g} \mathrm{L} \mathrm{L}^{-1}$.

Since many analytes are present at quite low levels in fuels, preconcentration techniques are occasionally required so that determination can be achieved more reliably. Teixeira et al. ${ }^{91}$ adjusted the $\mathrm{pH}$ of ethanol fuel samples to between 5 and 7.5 by the addition of the minimum amount of hydrochloric acid or sodium hydroxide. Aliquots of the sample $(10 \mathrm{~mL})$ were then passed through disks of P81 ion exchange cellulose chromatography papers that had been placed in polycarbonate filter holders. After drying, the papers were placed in an XRF sample cup and held in place by Mylar film. The papers were then analysed directly using EDXRF. Calibration was achieved by preparing standards in ethanol and passing these through filter papers in the same way as the samples. The analytes determined were $\mathrm{Cu}, \mathrm{Fe}, \mathrm{Ni}$ and $\mathrm{Zn}$. Parameters such as flow rate through the filter and sample volume were optimized. Although $1 \mathrm{~mL} \mathrm{~min}^{-1}$ was optimal (higher flow rates led to a significantly reduced signal for a $10 \mathrm{~mL}$ sample volume), a $2 \mathrm{~mL} \mathrm{~min}^{-1}$ flow rate was chosen as a compromise between sensitivity and sample throughput. A sample volume of $10 \mathrm{~mL}$ was adequate for the accurate determination of the analytes, although improved LOD could be obtained if higher sample volumes were used. This came at the cost of increased analysis time. Precision was approximately $3 \%$ RSD for all analytes and LOD were 13, 15, 15 and $12 \mu \mathrm{g} \mathrm{L}^{-1}$ for $\mathrm{Cu}, \mathrm{Fe}, \mathrm{Ni}$ and $\mathrm{Zn}$ respectively. The data obtained were compared with those obtained using a standard ICP-OES technique and were in excellent agreement. Another preconcentration technique prior to an atomic spectrometric determination of analytes in fuel ethanol was reported by Cortez and Pasquini. ${ }^{92}$ These authors used a flow injection-like manifold to drip sample onto a filter paper that was placed inside a 
small heated circular oven (called a ring oven). The droplets diffused by capillary action through the paper before they completely evaporated and formed a circular area of approximately $2 \mathrm{~cm}$ diameter. This enabled preconcentration factors of approximately 250 to be achieved. The papers were then analysed directly using LIBS providing the analytes $\mathrm{Cu}, \mathrm{Fe}$ and $\mathrm{Na}$ with LOD of $0.3,0.4$ and $0.7 \mu \mathrm{g} \mathrm{mL}{ }^{-1}$, respectively. Recoveries were $98 \pm 12,92 \pm 18$ and $109 \pm 13 \%$ for the same analytes. Although the ring oven technology was used more frequently between the 1950s and the 1970s, it still proved to be adequate for this application.

A flow blurring multi-nebulizer that had three liquid sample channels and one gas inlet was used by Aguirre et al. ${ }^{93}$ to introduce biodiesel and oil samples to an ICP-OES instrument. The materials were analysed using an online standard additions protocol that utilized aqueous standards. Comparison was made with a commercially available nebulizer that used organic-based standards for conventional standard additions. Both nebulizers introduced sample at a rate of $100 \mu \mathrm{L} \mathrm{min}{ }^{-1}$ to a cyclonic-style spray chamber. For the analysis of a multielemental Conostan organic standard, the flow blurring multinebulizer provided data that were in agreement within $\pm 3 \%$ of certified values. This was marginally better than those obtained using the commercial system, which agreed to within -5 to + $4 \%$. Short term ( 1 minute) and long term ( 2 hours) precision of the two systems were assessed with the multi-nebulizer being more precise in the short term especially at lower concentration. The long term stability test demonstrated conclusively that the multi-nebulizer (precision values at several wavelengths being between 1.5 and 2\% RSD) out-performed the commercial one (between 10 and 13\% RSD). This was attributed to the commercial system having to introduce organic material continuously which led to gradual build-up of carbon on the tip of the injector. Since the multi-nebulizer used aqueous standards to form standard additions online, the organic content entering the plasma was effectively decreased. Consequently, carbon build-up was also decreased. Characterization of the primary aerosols produced by the two nebulizers indicated that the multi-nebulizer produced droplets of less than $33 \mu \mathrm{m}$ in diameter, whereas the commercial one had a much coarser droplet distribution (less than $114 \mu \mathrm{m}$ ). Once optimized, the multi-nebulizer was applied to the analysis of biodiesel samples, with recovery data being close to $100 \%$ in all cases.

Da Silva et al. ${ }^{94}$ developed a method by which $\mathrm{Hg}$ in ethanol biofuel was determined using photochemical vapour generation with AAS detection. Sample $(25 \mathrm{~mL})$ was mixed with either formic acid $(1.3 \mathrm{~mL})$ or acetic acid $(2.5 \mathrm{~mL})$ and then diluted to $50 \mathrm{~mL}$ with water. The prepared sample was then pumped at a rate of $7 \mathrm{~mL} \mathrm{~min}^{-1}$ through a photochemical reactor comprising a tube, of dimensions $200 \mathrm{~cm}$ by $2 \mathrm{~mm}$, surrounding a mercury lamp operating at $254 \mathrm{~nm}$ and $15 \mathrm{~W}$. On emerging from the photochemical reactor, the sample entered a gas-liquid separator where the vapour was purged to AAS detection using an argon flow rate of $52 \mathrm{~mL} \mathrm{~min}^{-1}$. The sample flow rate, concentration of the organic acids and purge gas flow rate were all optimized to give the highest $\mathrm{Hg}$ signal. Although some $\mathrm{Hg}$ is observed in the absence of the organic acids, the signals increased rapidly to the optimal values before starting to decrease again. This decrease in signal at higher concentrations of organic acids was attributed to them absorbing the UV light, hence decreasing the amount of light available to form the $\mathrm{Hg}$ vapour. Determination of single standards of methyl- and ethylmercury indicated that there was no significant sensitivity difference between them and inorganic mercury. The method developed was, therefore, capable of total $\mathrm{Hg}$ determination. Calibration standards were prepared using bi-distilled ethanol to matrix match the samples as closely as possible. Recoveries of the analytes from spiked solutions of bioethanol ranged from 97 to $101 \%$ for samples fortified with formic acid and from 98 to $103 \%$ for those with added acetic acid. The detection limit was $0.05 \mathrm{ng}$ $\mathrm{mL}^{-1}$. The photochemical vapour generation system was described as being transferable to other detection systems, e.g., ICP-MS, which could provide significantly lower LOD. The system could, potentially, also be automated.

\subsection{Organic chemicals and solvents}

The challenges posed by the need for the analysis of art and historical objects are driving some significant developments in advanced analytical technology, as evidenced by the large number of papers dealing with applications in this field.

During this review period the development of combined analytical techniques, making use of both atomic and molecular information, together with spatial resolution is being increasingly reported. Synchrotron radiation sources are being employed to assist with the drive for more spectral and spatial resolution.

The use of data processing techniques to improve the quality and reliability of LIBS measurements is a now a significant feature of several new applications although matrix effects and interferences remain a significant barrier to the reliability of the technique.

Speciation of elements and an understanding of the molecular form of analytes features in a number of papers. The use of high resolution continuum source spectroscopy in molecular absorption mode is also reported.

2.2.1. The analysis of archaeological, cultural heritage and art objects. The analysis of culturally or historically significant artworks continues to feature prominently in the atomic spectrometry papers published during this review period. Multiple analytical technique, portable instrumentation and micro-capability are increasingly being employed to yield the maximum analytical information from these objects. Novel applications have been reported for a range of techniques to study paint layers on sculptures which have been restored and/or repainted several times over centuries. ${ }^{95}$ These include analysis by portable XRF, $\mu$-XRD and SEM-EDX, with $\mu$-Raman and $\mu$-FTIR being used in conjunction with the atomic techniques. This combination of molecular and atomic techniques has proved the only way to identify the pigments and $\mu$-Raman spectroscopy was particularly useful in characterising pigments which were difficult to determine by $\mu$-XRD. Multivariate analysis of XRF data has also proved beneficial as large amounts of spectroscopic data are acquired to extensively map the surfaces of paintings. ${ }^{96}$ This paper presents a strategy for the multivariate treatment of XRF spectral and hyperspectral data sets in situ to 
further support decisions on sampling strategy. This data treatment is able to account for inter-correlation among variables, which is important in elemental analysis. The spectral profiles were analysed directly by means of PCA. This approach facilitated a fast in situ interpretation of the results.

Alfeld et al. ${ }^{97}$ described how conventional imaging had revealed that a Rembrandt was painted on a re-used canvas and had covered the original image. However, the detail of this image could not be revealed. Vermillion ( $\mathrm{HgS})$ had previously been identified to have been used for the flesh tones of the lower, hidden painting, suggesting that element-specific XRF imaging may be useful to reveal the hidden image. Vermillion was used in a mock up of the overpainted image which was prepared for this work. XRF imaging of this mock painting was conducted using three different XRF imaging systems: a mobile $\mathrm{X}$-ray tube based system and two synchrotron-based systems. The experimental set ups are shown in some very informative diagrams in the paper and the negative images of the scanned mock painting are presented. Images from the three systems were compared and, surprisingly, the X-ray tube based scanner gave better results than the synchrotron scanner for $\mathrm{Ca}$ imaging, as the lower sensitivity was compensated for by the longer dwell time per point with the mobile scanner. For the heavier elements $(\mathrm{Hg}$ and $\mathrm{Pb})$, the synchrotron-based scanner was more than one hundred times more sensitive, permitting shorter acquisition times and smaller step sizes. This yielded much higher resolution and contrast. This work has provided supporting evidence that transporting the original Rembrandt to a synchrotron facility should be a useful activity to undertake.

Finely ground blue glass pigment used in a variety of painting techniques is known as smalt. In one study, smalt specimens dispersed in linseed oil were prepared and artificially aged in a thermostated oven and a water vapour saturated atmosphere to simulate the progressive deterioration of the pigment. The artificially aged specimens were compared with samples taken from a fifteenth century painting. A multi-technique approach including SEM-EDX, colorimetry, X-ray absorption spectroscopy and $a b$ initio calculations has been used to understand the progressive discoloration and to reveal its correlation with changes in the pigment structure. ${ }^{98}$ Colour space analysis has shown that the artificial aging results in the same discolouration as the naturally aged smalt. SEM-EDX data showed changes in K$\mathrm{Si}$ and $\mathrm{C}-\mathrm{Co}$ ratios on aging. Quantitiative XAFS data was in agreement with this observation. An integrated approach based on density function theory (DFT) and time dependent DFT (TDDFT) has been demonstrated to be a powerful tool for rationalising the spectroscopic properties of art heritage samples and to relate them to the atomic structure of cobalt coordination complexes. This technique was employed to understand the change of Co coordination from tetrahedral to octahedral. This was considered to be the major reason for discoloration due to the formation of octahedral water complexes.

A similar multi-technique approach was employed for the identification of fillers and pigments within a set of 19th century chromolithographs ${ }^{99}$ and an excellent example of a multi-analytical approach was described by Le Gac et al. in the determination of the authenticity of an 18th century Pascal
Taskin harpsichord. ${ }^{100}$ This paper presents a detailed characterisation of this musical instrument using EDXRF for the elemental analysis of the colour palette, optical and SEM imaging in both SE and BSE modes for the identification of the structural layers, SEM-EDX for the determination of the elemental composition of the inorganic compounds and $\mu$-FTIR spectroscopy for the determination of the binders. Thermally assisted hydrolysis methylation GC-MS (THM-GC-MS) was employed for the characterisation of the resinous layers. This work led to some important conclusions about the cleaning or enlargement of the instrument to encompass more notes. The layers of varnish served as chronological markers for this work. This paper presents some interesting photographic images of the layers of varnish and is an excellent illustration of the value of multispectral techniques for gaining maximum atomic and molecular information. In situ $\mu$-XRF in dispersive mode has been employed for the study of varnish on early musical instruments. ${ }^{101}$ This paper reported that the main pigments were $\mathrm{Fe}$ - and Mn-based materials. The presence of a Cr-based pigment in one instrument was a significant difference.

A review of instrumental approaches for studying historical inks ${ }^{102}$ comprises 76 references to papers published over the last 10 years and covers ICP-MS and GFAAS, LIBS, Mossbauer spectroscopy, X-ray techniques including TXRF, synchrotron X-ray sources, XANES, SEM-EDX, and PIXE. In addition to the atomic techniques, vibrational spectroscopy is included, covering IR and Raman spectroscopy and a range of sample presentation techniques including ATR and DRIFTS. The advantages of multi-technique studies are illustrated with suitable examples. Examples of the use of multispectral and hyperspectral imaging are given, and include one example of the use of NIR multispectral imaging with PCA to enhance the legibility of an iron gall ink which had been obscured by the presence of another ink. The reader may also find of interest a review of XRF in the investigations of cultural relics and archaeological finds. ${ }^{54}$ This review contains 111 cited references. Multiple techniques, including both atomic and molecular spectroscopies, have been employed for the comprehensive analysis of a sample of ink from an inkwell allegedly found at Qumran. Analysis of this ink was carried out by a combination of elemental and molecular spectroscopy, including GC-MS, proteomic analysis, PXRD, Raman, FTIR, LIBS and ICP-MS. This analysis revealed much detail about the manufacture of the inks, the processes involved and the organic and inorganic composition. The authors speculate that it is likely that this specific ink could be recognized if analysis is undertaken of inks on manuscripts from Qumran and other locations in Israel and the Middle East, raising the possibility that the provenance of inks on the Dead Sea Scrolls could be achieved. ${ }^{103}$

A second review proposes a multi-analytical strategy for the dating and authenticating historical manuscripts. ${ }^{104}$ This strategy sets out the order in which analysis is carried out and suggests that pigment analysis be carried out by Raman spectroscopy to distinguish the period, followed by PIXE ink analysis to determine ink homogeneity and, finally, radiocarbon dating of the support material, supplemented by ink-dating using scanning Auger microscopy. 
Analysis and imaging of overpainted images has traditionally been difficult. The higher spectral and spatial resolution of synchrotron radiation sources is advantageous in the analysis of historically and culturally significant objects and has recently been employed with some success for visualising images which have been overpainted. ${ }^{105}$ The authors report the presence of highly heterogeneous pigment mixtures, including $\mathrm{Cu}$ pigments beneath a 17th century Old Master. Scanning XRF was used to efficiently visualize the underpainting of a picture attributed to Rembrandt and this data made an important contribution to discussions of the authenticity of this painting. In another example of the use of synchrotron X-rays, Howard et al. employed high-definition XRF using the Maia XRF detector system on an X-ray microprobe, at the X-ray microscopy beamline at the Australian Synchrotron, Victoria, for the elemental mapping of paintings. ${ }^{106}$ This revealed the distribution of metals in the pigments underlying the brush strokes and enabled the characterisation of a wide range of pigments, thereby providing knowledge of the pigments used in an artists work and allowing inference of the dates of the pigments' manufacture and patterns of use. Single point XRF was extensively used because of the ease with which it enabled pigment discrimination. Scanning XRF had the capability to spatially resolve elemental distributions in two dimensions and mobile scanning XRF systems are routinely employed for the in situ analysis of artwork. Three dimensional scanning is possible with the depthsensitive technique of confocal XRF and speciation and the chemical environment of elements can be probed with XANES. One of the major disadvantages of scanning XRF was the slow pixel acquisition rate, usually around $1 \mathrm{~s}^{-1}$ therefore restricting the size or the resolution of the scan and making full composition analysis of a painting very time consuming. For example, the elemental mapping of Van Gogh's Patch of Grass took around 2 days. On a painting measuring $510 \mathrm{~mm}$ wide by $600 \mathrm{~mm}$ high and with appropriate choice of beam energy to minimize $\mathrm{Pb} \mathrm{L}$ shell fluorescence and Raman scattering, an elemental map was recorded in 22.5 hours. Compton scatter was found to yield information on the canvas weave. The experimental set up is described in detail in the paper. This synchrotron-based fast XRF mapping has provided a dramatic advance in the ability to extract information from high $\mathrm{Pb}$ containing artworks, with elemental mapping of tens of megapixels and a spatial resolution of tens of micrometers being demonstrated.

The high-resolution capability of synchrotron-based spectroscopy is also an advantage in studying photodegradation pathways. Speciation of Cd based pigments has been studied using synchrotron-based Cd L-lll-edge XANES at the Stanford synchrotron Radiation Light Source and SEM-EDS, SR- $\mu$ FTIR, XRF mapping and $\mu$-XANES mapping at the Cd L-lll-edge of paint cross-sections were carried out at the European synchrotron facility (ESRF). ${ }^{\mathbf{1 0 7}}$ Results showed that $\mathrm{CdCO}_{3}$ was substantially enriched in the off-white surface crust of the faded CdS-based paint, suggesting that the $\mathrm{CdCO}_{3}$ was present as an insoluble photodgradation product rather than solely as a filler or starting reagent. Oxalates and sulphates were also found to be concentrated at the alteration surface. Degradation of green Cu-based pigments has been determined by the combined techniques of
FTIR, XRD and XRF using a synchrotron source. ${ }^{108}$ The $\mathrm{Cu}$ pigments were identified as $\mathrm{Cu}$ acetates and chlorides and their degradation products were identified as a range of products including carboxylates, formats and oxalates.

In a different approach, high resolution nanoprobe $X R F$ mapping has yielded highly sensitive elemental mapping in submicron pigment particles. ${ }^{109}$ This has enabled a better understanding of the formulation of paints and their chemical reactivity. The high Renaissance marks a very significant period of artistic production in painting and artists of this epoch advanced aspects of art that continue to be researched today. Of significance is the use of the superimposition of multiple layers of paint to achieve the desired optical effects. The depth profiling of these paintings is an important aspect of their analysis and a laboratory-based confocal $\mu-X R F$ spectrometer (CXRF) has been applied to their non-destructive analysis. ${ }^{\mathbf{1 1 0}}$ Here, directly measured elemental depth profiles are compared to lateral scans and results of conventional analysis on crosssections removed from the paintings in the same locations. This comparison has enabled a better evaluation of the feasibility of using CXRF for depth profiling the paint layers. Limitations of the technique occur when the same element is present in adjacent layers, if the layers are $<10 \mu \mathrm{m}$ thick, or if the composition within a layer is highly heterogeneous. Layer sequences and later retouching were successfully determined.

The reader may find a review of the development and trends in synchrotron studies of ancient and historical materials useful. ${ }^{\mathbf{1 1 1}}$ This review contains 202 references and discusses the many advantages of synchrotron radiation, particularly the continuous tunability over a high energy range with a high intensity radiation.

The chemical analysis of very small samples is often of major interest in the analysis of artworks, particulate matter on filters and raw material impurities, amongst many others. Gazulla et $a l .{ }^{112}$ have developed WD XRF procedures specifically for the chemical analysis of small samples. Sample preparation protocols were determined to be critically important in ensuring that the required detection limits and precision levels were achieved. This paper evaluates three sample preparation procedures: beads for geological materials; beads for nongeological particulate material such as particulate matter on filters, glasses, frits and ceramic glazes and pigments; and pellets for the for the analysis of volatile elements in geological samples. Detection limits, quantification limits and measurement uncertainties were similar to those obtained when beads or pellets of around $0.5 \mathrm{~g}$ were prepared. However, in this paper, sample sizes 30 to 40 times smaller in the case of beads and 100 times smaller in the case of pellets were achievable.

LIBS is becoming the technique of choice for the remote detection of hazardous materials and is also generating interest in the analysis of historical paintings, where its capabilities are being enhanced by the use of multivariate data treatment. ${ }^{113}$ In this paper, LIBS was employed to study two fragments of prehistoric rock wall paintings found at two Brazilian sites. A time-resolved echelle based system using a Nd:YAG laser (1064 $\mathrm{nm}$ and $5.2 \mathrm{~ns}$ pulse) was constructed by the authors and depth profile composition of the samples was investigated by 
firing successive laser shots at each location on the samples, 8 shots covering different areas of the substrate and 8 covering pigmented areas. The rapid multi-element capability and the requirement for the consumption of very small amounts of sample make LIBS ideal for this type of investigation. The very complex nature of the emission spectrum and the care needed to distinguish between the layers of the sample necessitated the use of pattern recognition data handling procedures. PCA was employed to distinguish different materials, including painting materials and substrate materials, but also facilitated a study of the depth profiling of the rock painting. PCA also enabled an evaluation of the appropriate energy needed to reveal each layer, to identify the layers present and to determine their major components and also indicated which of the collected spectra better represented the composition of the pigment. This work was carried out using a surrogate sample of a multilayer painting on wood and the experimental conditions optimized on the surrogate were then used to analyse the various layers of the actual samples. A range of pulse energies were tested and the minimum power required to yield intense spectra whilst being capable of differentiating the layers of paint was determined. PCA analysis of the LIBS data revealed several features which enabled the authors to conclude important information on the archaeological sites, the elemental features indicative of certain sites and possible changes induced by geological processes at each site.

2.2.2. Remote analysis of harmful materials. LIBS continues to command much interest in many other application areas, not least of which is standoff detection of harmful materials. Standoff LIBS has been employed for the classification of trace residues as either hazardous (explosives, biological, etc) or non-hazardous and has been reviewed in previous review articles, for example last year's review. ${ }^{1}$ LIBS is very suitable for this type of analysis due to the requirement for minimal or no sample preparation, the minimal sample amount required to generate a usable emission spectrum and the ability to generate a signal in standoff mode, in some cases from a distance of many meters. However, correct classification of the analyte is very substrate dependent and varies in difficulty depending on what is underneath the residue. ${ }^{114}$ The success of classification can be further compromised if the substrate material has similar elemental composition to the residue. De Lucia and Gottfried $^{\mathbf{1 1 5}}$ have evaluated the coupling of multivariate analysis with standoff LIBS to determine the effectiveness of classifying thin explosive residue layers on a range of painted surfaces. They examine several organic substrates and determine the success of classification by LIBS using PLS-DA models with the goal to classify materials as either explosive or non-explosive irrespective of the substrate. A range of explosive materials were examined; cyclotrimethylenetrinitramine (RDX), trinitrotoluene (TNT) and a 36\% TNT, 63\% RDX and 1\% wax mixture. The mixtures (1-2 mg) were added to a range of car panels of various colours and paint types to give approximate surface coverage of around $10 \mu \mathrm{g} \mathrm{cm} \mathrm{cm}^{-2}$. A range of non-explosive materials, including road dust, sand, diesel, lubricating oil and fingerprint oil were also investigated. The standoff LIBS system and associated collection optics, together with optimized conditions are described in detail and employed a $1064 \mathrm{~nm} 10 \mathrm{~Hz}$ double pulse laser source with $5 \mathrm{~ns}$ pulse width. The sample area interrogated by the laser was estimated to be $5 \mathrm{~mm}^{2}$ at a standoff distance of $25 \mathrm{~m}$. PLS-DA models were constructed based on preselected emission intensities and ratios and amounted to 132 variables, significantly fewer than the variables required when using whole spectra models. This approach resulted in the correct classification of either explosive or non-explosive materials on seven car panels with a true positive rate of $98 \%$ and a false positive rate of $2.7 \%$. These rates were only marginally worse than data obtained using the whole spectra.

The influence of metal substrates on the detection of explosive residues was investigated by Gottfried. ${ }^{\mathbf{1 1 6}}$ The results demonstrated that correct classification of RDX residue when applied to a range of metal substrates is feasible when PLS-DA is used. When limiting the spectral data examined to the features pertaining to RDX, a true positive rate of $96.5 \%$ and a false positive rate of $1.0 \%$ for RDX classification was achieved on a range of metal and metal alloy surfaces. PLS-DA was used by Wang et al. to distinguish explosives from plastics. ${ }^{117}$ They demonstrated that LIBS could discriminate explosives from seven types of plastics, although only one explosive was tested.

An alternative chemometric approach for identifying explosives by LIBS, employing machine learning classifiers, has been developed by Moros et al. ${ }^{118}$ A variety of organic residues, both explosive and potential interferent compounds located on a support surface were surveyed and their spectral responses determined. Relationships between the optical emission characteristics of residues and their hazardous nature have been extracted. By using the original intensities of the most relevant emission lines ( $\mathrm{C}, \mathrm{CN}, \mathrm{C}_{2}, \mathrm{H}, \mathrm{N}$ and $\mathrm{O}$ ), supervised learning methods (SLMs) were employed to cope with the complex and subtle differences between the LIBS spectra of explosive residues and potential interferents. Relevant spectral features were extracted from a training set of data and, on the basis of 2-D scatter plots, the variables were then processed to search for trends serving to differentiate between classes. This approach is described in detail in the paper and was shown to be capable of discriminating a range of organic explosive residues from several potential interfering materials when placed on the surface of an aluminium support. False positives and false negatives were better than $5 \%$. A slightly different approach by Fernandez-Bravo et al. ${ }^{119}$ involved the selective sampling of explosive residues on organic substrates. Since ablation and subsequent plasma formation are sensitive to the beam focal conditions and the pulse energy at the surface, the choice of an appropriate set of experimental conditions enabled the surface sensitivity to be controlled. This enabled a selective analysis of the residue in the absence of any spectral contribution from the organic substrate. 2-mononitrotoluene, 2,6-dinitrotoluene and 2,4,6-trinitirotulene were used as the model residues and nylon and Teflon were used as model substrates. Selective sampling of the residues on these substrates was successful in all cases where the plasma formation threshold of the residue was substantially different to the substrate.

It is well known that two-component explosives consisting of metal particles can yield more than twice the energy of molecular 
explosives alone. ${ }^{120}$ In this paper, LIBS was employed to probe some fundamental mechanisms occurring within the plasma, which is thought to be a useful model for reactions occurring within explosions. The plasma generated in LIBS experiments was employed to study the chemical reactions between metallic nanoparticles and RDX. Double pulse LIBS under argon was used to generate the plasma and exclude atmospheric nitrogen. Background corrected emission intensities for atomic and molecular species relevant to RDX were tracked over the first $10 \mu \mathrm{s}$ of the laser induced plasma lifetime. Bremsstrahlung background features were subtracted to give relevant emission lines for $\mathrm{C}, \mathrm{H}$ and $\mathrm{N}$. The molecular species exhibited very different time-dependent behaviour. Similarities between the chemistry within an explosion and a plasma are discussed in this paper. The time resolved atomic and molecular emissions of aluminium nanoparticles and organic species from the RDX, within the plasma were measured to gain an understanding of this chemistry. This confirmed that the presence of aluminium promotes the ejection process of carbon from the intermediate RDX reaction products and assumptions made in previous kinetic studies were shown to be invalid.

Least squares support vector machines (LS-SVM) are proposed as a means of improving variability in LIBS analysis so that the applicability of the technique may be extended to quality assurance and process monitoring in pharmaceutical production. ${ }^{121}$ Statistically significant improvements in the classification of pharmaceutical samples were demonstrated and this was due to the ability of this non-linear classification framework to address the variability in ablation of the sample and self absorption within the plasma. The authors claimed a significant improvement in correct allocation rate and a concomitant reduction in misallocation, even when samples not included in the training set were analysed, as compared with SIMCA and PLS-DA. The authors propose that this method of classification is sufficiently robust for the screening for counterfeit pharmaceuticals. Further work is required, involving the selection of spectral features, rather than the inclusion of the whole spectrum, which the authors postulate may worsen the model. The ultimate goal of this work is to enable the use of LIBS in the pharmaceutical industry as a routine quality assurance technique. In a further pharmaceutical application, LIBS was evaluated as a technique for the rapid screening and quality control of anti-diabetic tablets. ${ }^{122}$ Two APIs, metformin and glybenclamide were the compounds of interest. Chlorine concentration was estimated by measuring the ratio of the emission lines of chlorine to bromine, where bromine was added as an internal standard. Results were compared with those obtained by HPLC with UV detection and recoveries were $95-101 \%$. The reviewer notes that in the case of metformin the chlorine atom is present in the counter ion and not covalently bonded to the molecule. This procedure, therefore, relies on the presence of metformin as the hydrochloride. Hence, any changes such as the loss of $\mathrm{HCl}$ on storage may result in low recoveries but this was not examined in the paper.

2.2.3. Speciation. Auranofin (2,3,4,6-tetra-O-acetyl-1-thio- $\beta$ D-glucopyranosato-S-(triethyl-phosphine)gold) has been used in the treatment of rheumatoid arthritis since the 1970s but despite its long usage in this treatment its mode of action is still not fully understood. It undergoes ligand exchange reactions in vivo to generate reactive metabolites. Albert et al. ${ }^{123}$ present the development of LC-ESI-MS and LC-ICP-MS for the speciation of Auranofin and its thiol drug-protein adducts to develop an understanding of the reaction pathway of the drug. Previous studies with ${ }^{197} \mathrm{Au}$ Mössbauer (cited in this paper) have proposed a monomeric Auranofin with linear $\mathrm{S}-\mathrm{Au}-\mathrm{P}$ coordination. Proton and carbon NMR confirmed the proposed binding environment of the gold atom. Early attempts with ESIMS showed several gold species in the mass spectrum but did not preclude the possibility that these were formed in the ESI interface. In this paper, data from both LC-ESI-Orbitrap-MS and LC-ICP-MS has shown that the monomeric Auranofin is eluted from the column and the other species are formed in the ESI interface. It was shown that monomeric Auranofin is the main compound to undergo exchange reactions with added thiol ligands. Adduct formation of the gold species with glutathione and human serum albumin was simulated successfully in vitro and analysed by LC-ESI-MS and LC-ICP-MS. This showed that whilst the thiol ligand is displaced, the remaining triethylphosphine gold structure may covalently bind to either glutathione or human serum albumin. Moreover, oxidation of the phosphine ligand by disulphides occurs in both reaction mixtures. Phosphine oxide is the resulting product in both cases. Reactions of Auranofin with the thiols were quantitatively traced over several days by ICP-MS. This data enabled a potential reaction pathway of Auranofin with the two endogenous thiols to be proposed.

The S-containing drug metabolites, ethacrynic acid and tiotropium bromide, from in vitro and in vivo metabolism studies have been determined by LC-ICP-MS. ${ }^{124}$ The purpose of the LC separation was to minimize interference from diatomic oxygen. Quantitation was by monitoring $\mathrm{SO}^{+}$at $48 \mathrm{Da}$, which is free from isobaric interferences. The sensitivity of this species, along with plasma stability, is significantly influenced by mobile phase composition and various correction strategies were proposed, including calibration curves based on different solvent concentrations generated by FIA and post-column gradient compensation. Results obtained have shown that quantitation was successful regardless of the mobile phase composition once these correction strategies were applied. S-containing metabolites were readily distinguishable from endogenous S-containing compounds in animal studies and the authors propose this as a viable alternative to the use of radio labelled compounds or the necessity for having synthetic standards of the anticipated metabolites. Complementary analysis by LC-MS-MS was useful in determining the number of S-atoms in each metabolite.

Meglumine antimonite is an API used in the treatment of Leishmaniasis, a tropical disease caused by a parasite. The API is mainly $\mathrm{Sb}(\mathrm{v})$, present as a complex with $\mathrm{N}$-methylglucamine. $\mathrm{Sb}$ (III) may be present as a manufacturing impurity and this is likely to be complexed. As $\mathrm{Sb}(\mathrm{III})$ is more toxic than $\mathrm{Sb}(\mathrm{v})$, the determination of these $\mathrm{Sb}$ species is, therefore, required. The concentrations of $\mathrm{Sb}$ (III) are difficult to determine accurately and Seby et al. ${ }^{125}$ have developed two independent procedures for the determination of Sb species. In the first procedure, an 
anion exchange IC separation was coupled with ICP-MS. This was cross-checked with a differential pulse polarography (DPP) method, which could also be used routinely on the production site. Prior to detection, it was necessary to generate the free $\mathrm{Sb}$ species and this was achieved by diluting the samples in $\mathrm{HCl}$. Air was excluded to minimize $\mathrm{Sb}$ redox reactions, although the reviewer believes that $\mathrm{Sb}(\mathrm{III}) / \mathrm{Sb}(\mathrm{v})$ equilibria may still be an issue. The authors address this to a certain extent through judicious choice of $\mathrm{HCl}$ concentration. Good agreement was achieved for $\mathrm{Sb}$ (III) between both procedures.

A review of ICP-MS in drug development containing 166 references is included for the interested reader. ${ }^{126}$ This review covers aspects of bioanalysis and includes hyphenation with LC and CE. A useful review of hyphenated techniques as tools for speciation analysis of metal-based pharmaceuticals is also included. ${ }^{127}$ This review contains 179 references and includes method development, advantages and limitations of separation modes including HPLC, CE and GC and detection by ICP-MS and ESI-MS.

Fluorine has been determined in toothpaste by measuring the molecular absorption of aluminium mono fluoride using a highresolution continuum source and nitrous oxide-acetylene FAA. ${ }^{128}$ The paper discusses the optimisation of the instrumental parameters, with particular emphasis on the concentration of the molecule-forming reagent which was $\mathrm{Al}$ in this case, as linearity depends strongly on this concentration. Detection limits for Al were $5.5 \mathrm{mg} \mathrm{L}^{-1}$ based on $3 \delta$ and $n=10$. Results were in line with those claimed by the manufacturers. The advantage of this procedure is that the $\mathrm{F}$ in monofluorophosphate is accurately determined and this is not the case when using IC or ISE due to the non-quantitative nature of the release of $\mathrm{F}$ in solution. The authors claim that standard addition calibration is desirable to minimize interferences from competitive reactions with other matrix constituents, however, the reviewer urges caution as the validity of this claim was not tested in the paper.

Graphite furnace continuum source molecular absorption spectrometry (HR-CS-MAS) has been employed for the determination of bromine in aqueous solution as well as in organic solvents. ${ }^{129}$ In this case, the bromine was determined by the molecular absorption of $\mathrm{CaBr}_{2}$. The claimed linear range was 6 orders of magnitude (pg to $\mu \mathrm{g}$ ) with a detection limit of $78 \mathrm{pg} \mathrm{Br}$ and good recoveries for both inorganic and organic salts. The procedure was very tolerant to interferences from $\mathrm{Al}, \mathrm{Cl}, \mathrm{F}, \mathrm{Fe}$ and I and was employed to determine polybrominated flame retardants (PFBRs).

2.2.4. Applications requiring minimal sample preparation. The technique of TXRF is virtually unaffected by matrix effects, is very sensitive, with ppb level detection limits being commonly achieved and can require little sample preparation. As such it is potentially a very useful technique in many application areas, including pharmaceutical analysis. Pd and other metals are extensively used in pharmaceutical small molecule drug substance processes as catalysts and must be removed prior to release of the active pharmaceutical ingredient (API). Shaw et al. ${ }^{130}$ have evaluated TXRF instruments from two vendors (Bruker AXS, Technos) to establish whether TXRF can be used to accurately, precisely and sensitively determine Pd in pharmaceutical drug substances and whether it can be used to screen for other pharmaceutically relevant metals. They also looked at the advantages of TXRF as compared with ICP-MS for these analyses. Six drug substances (triprolidine $\mathrm{HCl}$, diphenylhydramine $\mathrm{HCl}$, chlorpheniramne maleate, pseudoephedrine $\mathrm{HCl}$, ephedrine sulphate and scotamine $\mathrm{HBr}$ ) were analysed by both instruments, each configured to access either Pd-L shell (binding energy $3.61 \mathrm{keV}$ ) or the Pd-K shell $(24.35 \mathrm{KeV})$, to determine linearity, accuracy and precision for $\mathrm{Cr}, \mathrm{Fe}, \mathrm{Pd}, \mathrm{Pt}$ and $\mathrm{Rh}$. Interferences from $\mathrm{Ar}, \mathrm{Cl}$ and $\mathrm{S}$ were checked at various fluorescence lines. Internal standardisation was found to be important to remove systematic errors resulting from variations in spotting and drying of the samples. The results for $\mathrm{Pd}$ on each instrument in their different configurations are shown, with detection limits between $0.001 \mu \mathrm{g} \mathrm{ml}$ and $3 \mu \mathrm{g} \mathrm{ml}^{-1}$ and performance being very dependent upon the instrument configuration. The difference between the detection of Pd-L and Pd-K lines was very important and instruments with access to $\mathrm{Pd}-\mathrm{K}$ lines were found to be most effective in determining Pd in APIs. The primary advantages of TXRF are its ability to accept organic solvent diluents, its ease of use and low operational cost when compared with ICP-MS. The authors concluded that TXRF based upon Pd detection is an ideal screening platform. However, an instrument with multiple sources would open the technique to wider use but at greater cost. TXRF has also been employed for the determination of $\mathrm{Au}$ and $\mathrm{Pt}$ containing active ingredients in metallodrugs at the ppb level in a cell suspension. ${ }^{\mathbf{1 3 1}}$

Mercury continues to pose significant analytical challenges and in one paper the use of a miniaturized micro-plasma based optical emission system has been developed for mercury determination. Photochemical vapour generation (PVG) was coupled with optical emission spectrometry using dielectric barrier discharge (DBD) as an excitation source. ${ }^{\mathbf{1 3 2}}$ With the exception of dilution and acidification with formic acid, thimerosal in vaccines was determined with no sample pretreatment, thereby eliminating the need for strong oxidising and reducing agents. Volatile $\mathrm{Hg}$ was generated by PVG and excited by the DBD plasma. The DBD micro-plasma source was operated under low power $(<18 \mathrm{~W})$ and low argon consumption

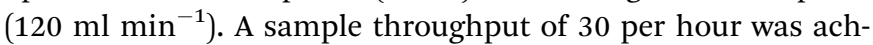
ieved with sample presentation by flow-injection. Operational parameters and potential interferences were investigated and described thoroughly in the paper, as was the flow-injection manifold. Detection limits for inorganic $\mathrm{Hg}^{2+}$ and thiomersal as $\mathrm{Hg}$ were $0.19 \mu \mathrm{g} \mathrm{L}^{-1}$ and $0.17 \mu \mathrm{g} \mathrm{L}^{-1}$ respectively. The detection limit was defined as $3 \sigma / m(n=10)$ and $m$ is the slope of the calibration data, well below the levels determined in 4 of the 6 different vaccines evaluated. Results were checked against an ICP-MS procedure and excellent agreement was found. This technology could be extended to other hydride forming elements such as As, Cd, Se and some transition elements including $\mathrm{Fe}$, and $\mathrm{Ni}$, although the authors do not show data for these elements. The low power and gas consumption should also prove useful in cases where portability is a requirement. Matrix assisted PVG with ICP-MS detection has also been 
employed for the determination of $\mathrm{Hg}$ in alcoholic drinks, again with no added reducing agent. ${ }^{133}$

Analysis of organic matrices by ICP techniques is challenging and has been addressed in one paper for the determination of $\mathrm{Cd}$ and $\mathrm{Pb}$ by the introduction of sub- $\mu$ l volumes of analyte into the plasma via a microfuidic chip within a FIA system. ${ }^{134} 52 \%$ ethanol was employed as a surrogate for distilled spirits and the effects of injection of varying volumes on the plasma stability and mass spectral interference from carbon were investigated. With a sample volume of $0.8 \mu \mathrm{l}$ there were no carbon deposits and the plasma stability was not affected. Further reduction of sample volume to $0.3 \mu \mathrm{l}$ resulted in no detectable spectral interference from carbon. Calibration was by external standardisation with aqueous standards and results were in good agreement with those after the matrix was destroyed by microwave digestion. It is often desirable to directly analyse solid samples with no dissolution step, to minimize the potential for contamination or analyte loss, especially where very limited amounts of material are available. One example of this is the forensic analysis of automotive paint samples found at crime scenes, especially hit and run cases. Solid sampling electrothermal vaporisation coupled with ICP-OES has been developed for the discrimination of automotive paint samples. ${ }^{135}$ In this paper, 18 samples of paint fragments were collected from 6 red car wrecks, sampled from the front, side and back. For each sample, 2 replicates of 0.8 to $2.0 \mathrm{mg}$ of paint were weighed directly onto a graphite ETV boat which was placed into the ETV furnace for vaporisation at $2200{ }^{\circ} \mathrm{C}$. The vapour was then transferred into the ICP in an Ar carrier containing $\mathrm{Cl}_{2} \mathrm{~F}_{2} \mathrm{C}$ reactant gas. Numerous elements were monitored but the very high sample introduction efficiency meant that several elements saturated the detector and the less sensitive elements were chosen. Of these, only 13 elements which showed significant variation between each paint sample were used. Normalisation of the emission of each chosen element to an Ar line was carried out, followed by integration of the signal. The data matrices, comprised of the integrated areas divided by the sample mass for each of the elements, were then subjected to PCA, cluster analysis and LDA. After identifying the major contributing variables by PCA, LDA was performed and showed that a combination of three or four variables from $\mathrm{Cr}, \mathrm{Pb}, \mathrm{Sn}$ and $\mathrm{Zn}$ correctly assigned each of the red paint samples to the correct source car. The correct assignment of the paint samples relies strongly upon the precision of the analysis and typical RSD values of between $6 \%$ and $12 \%$ were sufficient.

In a novel combination of electrothermal sampling of solids with HRCSAAS, the $\mathrm{Pb}$ content in lipstick was determined. ${ }^{136}$ The optimized procedure enabled around 0.25 to $2 \mathrm{mg}$ of sample to be loaded directly onto the platform and interference-free determination of $\mathrm{Pb}$ was carried out after calibration with aqueous standards, with an LOD of around $21 \mathrm{pg}$. In comparison of the results with microwave-assisted digestions no statistically significant differences were found.

2.2.5. Applications requiring analyte extraction or sample dissolution. Determination of trace impurities in pharmaceutical preparations is an important regulatory requirement. Microwave-induced combustion (MIC) has been applied for the digestion of APIs and subsequent determination of bromine and iodine by ICP-MS. ${ }^{137}$ Ten APIs including amoxicillin, atenolol, clavulanic acid, clonazepan, diltiazem, haloperidol, imipramine, nimesulide, propranolol and sodium diclofenac were digested by MIC. Combustion of $500 \mathrm{mg}$ of each API was achieved in $<30 \mathrm{~s}$ using 20 bar oxygen. Ammonium carbonate $(50 \mathrm{mM})$ was the absorbing solution for both analytes and a 5 minutes reflux step was included to maximize recoveries. Recoveries for $\mathrm{Br}$ and I from CRMs, after going through the same digestion procedure were 95 and $97 \%$ respectively. Residual carbon was determined by ICP-OES and was found to be $<500 \mathrm{mg} \mathrm{L}^{-1}$, an important consideration when determining the analytes by ICP-MS. Detection limits for Br and I by ICP-MS were $0.001 \mu \mathrm{g} \mathrm{g}^{-1}$ and $0.02 \mu \mathrm{g} \mathrm{g}{ }^{-1}$, respectively, compared with $4.2 \mu \mathrm{g} \mathrm{g}^{-1}$ and $0.3 \mu \mathrm{g} \mathrm{g}^{-1}$ by IC.

Guidelines for elemental impurities and novel analytical procedures are being adopted by the United States Pharmacopeia. In response to this, optimized sample preparation and quadrupole ICP-MS measurement protocols for the determination of elemental impurities in pharmaceutical products have been developed. ${ }^{138}$ This paper outlines the optimisation of two sample preparation procedures for organic pharmaceutical excipients, one relying on direct dissolution and the other, on microwave-assisted acid digestion. Optimisation concentrated on the reliable determination of $\mathrm{Hg}$, $\mathrm{Pd}$ and Os. Recoveries and concentrations were determined in two samples by ICP-MS with collisional removal of polyatomic interferents. Detection limits were an order of magnitude lower than the proposed limit concentrations for parenteral administration. Recoveries of 93.8 and $109.9 \%$ were achieved when standard addition calibration was employed.

A classification of a range of sorbents and SPE procedures for the determination of Au, Pd and Pt in different sample types has been proposed in a review article containing 214 references ${ }^{139}$ The authors classify the sorbents by their sorbent mechanisms, e.g., complex formation, ion exchange, adsorption, ion or molecular-imprinted polymers. These latter two sorbent classes are further divided, based on the kind of monomeric unit used to build the polymer, and the functional group responsible for imparting the characteristics (selectivity and capacity) of the polymer. Advantages of chemically modified sorbents, S-containing sorbents, together with microwave pretreatments and quantification by means of flow-injection approaches are discussed. Detection techniques reviewed include ICP-OES, ICPMS, ETAAS and NAA.

Determination of Se by solid phase micro extraction (SPME) coupled with a graphite furnace atomisation and detection by GC-MS is described by da Silva et al. ${ }^{140}$ Various factors affecting the derivatisation and extraction of Se(Iv) by SME-GF were evaluated including the acid type and concentration, the concentration of the derivatising agent, temperature and the extraction and derivatisation times and temperatures. $\mathrm{Se}^{\mathrm{IV}}$ detection limit was $0.37 \mu \mathrm{g} \mathrm{L}^{-1}$ defined as 3.3 multiplied by the ratio of standard deviation of the $y$-intercept to the slope. The determination of total Se in a CRM of water resulted in a $97 \%$ recovery, and $101 \%$ for plankton. Reduction of total Se to $\mathrm{Se}^{\mathrm{IV}}$ was required for quantitative recovery in the water and the 
plankton samples but when a drug sample was analysed the reduction step did not make a significant difference, suggesting that all the Se present in the drug was present as $\mathrm{Se}^{\mathrm{IV}}$. The paper references previous work coupling SPME with GF and contains useful diagrams illustrating the procedure, with extraction into the SPME fibre occurring within the GF. The fibre was then desorbed in the injector of the GC-MS system. Only $99 \mu \mathrm{l}$ of derivatized solution was required for this procedure, compared with $7 \mathrm{ml}$ for a conventional SPME procedure.

Multiwalled carbon nanotubes have been employed in SPE of a range of metals ( $\mathrm{As}, \mathrm{Bi}, \mathrm{Cd}, \mathrm{Hg}, \mathrm{Pb}$ and $\mathrm{Ti}) .{ }^{141}$ Extraction from cosmetic creams and detection by ICP-OES gave good recoveries and ppb detection limits.

Ultrasound assisted extraction has been notable by its absence in the recent literature with one exception for the determination of trace $\mathrm{Cr}$ and $\mathrm{Mn}$ in pharmaceutical formulations. ${ }^{142}$ Determination of these two elements was by ETAAS. The procedure was optimized for sonication power, extraction time and concentration of $\mathrm{HCl}$ in the extractant solution, together with mass of sample. The optimized extraction procedure gave quantitative recovery of the two elements from a range of pharmaceutical formulations including ciprofloxacin, cephalexin, azithromycin, amiodipine and methionine.

It is often desirable to directly analyse solid samples with no dissolution step, to minimize the potential for contamination or analyte loss, especially where very limited amounts of material are available. One example of this is the forensic analysis of automotive paint samples found at crime scenes, especially hit and run cases. Solid sampling electrothermal vaporisation coupled with ICP-OES has been developed for the discrimination of automotive paint samples. ${ }^{135}$ In this paper, 18 samples of paint fragments were collected from 6 red car wrecks, sampled from the front, side and back. For each sample, 2 replicates of 0.8 to $2.0 \mathrm{mg}$ of paint were weighed directly onto a graphite ETV boat which was placed into the ETV furnace for vaporisation at $2200{ }^{\circ} \mathrm{C}$. The vapour was then transferred into the ICP in an $\mathrm{Ar}$ carrier containing $\mathrm{Cl}_{2} \mathrm{~F}_{2} \mathrm{C}$ reactant gas. Numerous elements were monitored but the very high sample introduction efficiency meant that several elements saturated the detector and the less sensitive elements were chosen. Of these, only 13 elements which showed significant variation between each paint sample were used. Normalisation of the emission of each chosen element to an Ar line was carried out, followed by integration of the signal. The data matrices, comprised of the integrated areas divided by the sample mass for each of the elements, were then subjected to PCA, cluster analysis and LDA. After identifying the major contributing variables by PCA, LDA was performed and showed that a combination of three or four variables from $\mathrm{Cr}, \mathrm{Pb}, \mathrm{Sn}$ and $\mathrm{Zn}$ correctly assigned each of the red paint samples to the correct source car. The correct assignment of the paint samples relies strongly upon the precision of the analysis and typical RSD values of between $6 \%$ and $12 \%$ were sufficient.

In a novel combination of electrothermal sampling of solids with HRCSAAS, the $\mathrm{Pb}$ content in lipstick was determined. ${ }^{136}$ The optimized procedure enabled around 0.25 to $2 \mathrm{mg}$ of sample to be loaded directly onto the platform and interference-free determination of $\mathrm{Pb}$ was carried out after calibration with aqueous standards, with an LOD of around $21 \mathrm{pg}$. In comparisons of the results with microwave-assisted digestions no statistically significant differences were found.

\subsection{Inorganic chemicals and acids}

There has been a greater emphasis on novel papers in this year's review. Much of the current research into cements and concretes has focussed on aspects other than their analysis. Consequently, this section has been removed. Instead, it has been replaced by sections entitled "Remote analyses of inorganic materials" and "coupled techniques".

2.3.1. Coupled techniques. There have been several applications published that involve the use of a separation technique coupled with an atomic spectrometric detector. Such a coupling would normally be described as speciation analysis and it enables the different oxidation states or different chemical forms of an analyte present in a sample to be determined. The large majority of these applications are outside the remit of this update and are, instead, reviewed in a separate update. There are, however, some applications that are directly relevant to this review and these will be discussed below.

The metal to halide mole ratios in platinum complex ions have been determined by van Wyk et al. ${ }^{143}$ who used HPLC-ICP-OES to separate the species and then detect $\mathrm{Br}, \mathrm{Cl}$ and $\mathrm{Pt}$. The complex ions took the form of $\left[\mathrm{PtCl}_{6-n} \mathrm{Br}_{n}\right]^{2-},\left[\mathrm{PtCl}_{4}\right]^{2-}$ and $\left[\mathrm{PtCl}_{6}\right]^{2-}$ where $n$ could range from 0 to 6 . The different species were separated using a reversed phase, octadecylsilane column using the ion pairing reagent tetrabutylammonium nitrate and the analytes were detected at $177.708,134.724$ and $148.845 \mathrm{~nm}$ for $\mathrm{Pt}, \mathrm{Cl}$ and $\mathrm{Br}$ respectively. All nine possible species were detected when monitoring Pt emission but, of course, only eight peaks were observed for $\mathrm{Cl}$ because no peak would appear for the species $\left[\mathrm{PtBr}_{6}\right]^{2-}$. Similarly, the $\mathrm{Br}$ chromatogram produced only seven peaks since no emission signal was observed for $\left[\mathrm{PtCl}_{6}\right]^{2-}$ and $\left[\mathrm{PtCl}_{4}\right]^{2-}$. It was noted though, that resolution between some of the species was quite poor which could lead to inaccuracies in some of the mole ratios calculated. Optimization of the separation process did yield sufficiently good resolution, albeit by ESI-MS detection. The eluent used for the separation was $55 \%$ acetonitrile, $45 \%$ water, containing $9 \mathrm{mM}$ tetrabutylammonium nitrate. The other problem reported was that very large concentrations of species had to be injected so that the halogens could be determined. This was because they have a particularly poor LOD using ICP emission techniques. Despite these problems, the authors did report that the method was the first developed to determine them in such a way.

Several applications have utilized capillary electrophoresis (CE) coupled with ICP-MS detection. In one such application, Varenne et al. ${ }^{144}$ used CE-ICP-MS to determine metal affinities and binding constants of phosphorylated ligands. A standard $80 \mathrm{~cm} \times 70 \mu \mathrm{m}$ capillary was treated with methanol, followed by water and then dilute $\mathrm{NaOH}$. The inner surface was then coated with a $0.1 \% \mathrm{~m} \mathrm{~m}^{-1}$ solution of polybrene containing a large excess of the chloride form of the anion exchange resin AG MP-1 present in a formic acid/sodium chloride separation buffer. The 
operating conditions for the CE-ICP-MS system were listed in the paper and a specialist low-flow nebulizer was used. The coupling between the CE and ICP-MS instruments was via a home-made sheath-flow interface, the principles of which were also given. Fifteen phosphorylated compounds, including phosphine oxides, thiophosphines, phosphonates and phosphinates, were separated in less than one hour and using less than $5 \mathrm{ng}$ of material. The P-containing compounds could be classified according to their Eu ${ }^{\mathrm{III}}$ binding affinity in a hydroorganic medium (sodium formate, $\mathrm{pH}$ 3.7, water-methanol in a 90-10 mix). By varying the total Eu concentration, complexation constants could be determined according to a sequential multiple run strategy. The data obtained were in excellent agreement with the values obtained using UV-Vis spectrophotometric titrations. The identification and quantification of potential metabolites of Gd-containing contrast agents used for magnetic resonance imaging were reported by Telgmann et al. ${ }^{145}$ who used CE-ICP-MS as well as CE-ESI-MS for the task. In particular, the products formed after electrochemical oxidation were studied. The ESI-MS elucidated the structure of the products whereas the ICP-MS was better suited to quantifying the species. Two products were identified from the oxidation of the compound gadopentate. The material underwent an $\mathrm{N}$-dealkylation reaction. One of the metabolites was unstable and underwent further decomposition. Under the conditions used in the experiment, $8.7 \%$ of the original compound was oxidized.

It is well known that when CE or any ultra-low flow method is coupled with an atomic spectrometric detector, the suction effect exerted by the nebulizer can destroy the chromatography. A paper by Cheng et al. ${ }^{\mathbf{1 4 6}}$ discussed the elimination of this problem when using microchip electrophoresis coupled with ICP$M S$ (MCE-ICP-MS) by using porous monolithic plugs. The system was very complex, but involved a microchip, a demountable capillary micro-flow nebulizer, a heated single pass spray chamber, a negative pressure sampling device, a high voltage power supply, a syringe pump and the ICP-MS instrument. Full details and a schematic diagram of the setup were given in the paper. Full details were also given for the preparation of the chip and of the porous polymer plugs that were inserted into the chip to prevent the suction effects. Optimization of the electrophoresis was undertaken and this included a study of the flow rate, the buffer system used, the voltage applied and separation paths of different length. The eluent from the chip was transferred to the nebulizer by $0.1 \% \mathrm{HNO}_{3}$ containing $10 \mu \mathrm{g} \mathrm{L}^{-1} \mathrm{In}$ as an internal standard at a flow rate of $10 \mu \mathrm{L} \mathrm{min}{ }^{-1}$. The system was applied to the determination of iodide and iodate $\left(\mathrm{I}^{-}\right.$and $\left.\mathrm{IO}_{3}{ }^{-}\right)$in table salt. The sample was introduced to the system and the two analytes were separated using an electric field of $857 \mathrm{~V} \mathrm{~cm}^{-1}$ and a running buffer of $10 \mathrm{mM}$ borate ( $\mathrm{pH}$ 9.2). The separation path was only $15 \mathrm{~mm}$ long and this enabled the species to be separated within $25 \mathrm{~s}$. Since only very low volumes were introduced to the system, the absolute LOD were impressive, being 0.12 and $0.13 \mathrm{fg}$ for $\mathrm{I}^{-}$and $\mathrm{IO}_{3}{ }^{-}$respectively. Precision of the migration times was in the range $1.1-1.6 \%(n=10)$ and the analytical precision was $2.5-$ $2.8 \% \mathrm{RSD}$. The levels of $\mathrm{I}^{-}$and $\mathrm{IO}_{3}{ }^{-}$in the table salts were in accordance with the labelled values. Spiking of the samples yielded recoveries in the range $92-105 \%$.

2.3.2. Forensic applications. The relevant papers in this section of the review concentrated on the forensic analysis of gunshot residue. In one, by Latzel et al., ${ }^{147}$ milli-XRF was used to provide an elemental map of the area of bullet impact and this could then be used to estimate the approximate shooting distance. A numerical tool was presented that displayed the data and enabled a more reliable estimate of shooting range to be made. Despite the advances made, the authors admitted that some problems still existed and these included when there were only a few residue spots spread over a large distance or when the material on which the residue is deposited provides a highly scattering background. The paper presented many of these problems and concluded that the spectral interpretation could not be fully automated since an experienced analyst was required to verify some of the points. A second application, this time by Abrego et al., ${ }^{148}$ described the development of a LA-ICPMS method for the unambiguous characterization of gunshot residue. Tape lifts were used to collect the residues from skin surfaces and these were then analysed for their ${ }^{121} \mathrm{Sb},{ }^{137} \mathrm{Ba}$ and ${ }^{208} \mathrm{~Pb}$ content. Several other analytes, e.g. ${ }^{27} \mathrm{Al},{ }^{29} \mathrm{Si},{ }^{33} \mathrm{~S},{ }^{35} \mathrm{Cl},{ }^{39} \mathrm{~K}$, ${ }^{44} \mathrm{Ca},{ }^{57} \mathrm{Fe},{ }^{60} \mathrm{Ni},{ }^{63} \mathrm{Cu},{ }^{66} \mathrm{Zn}$ and ${ }^{118} \mathrm{Sn}$, were also monitored to obtain additional information. Numerous different firearms, calibres and ammunition were used during the study. It was demonstrated that the LA-ICP-MS approach could be a real alternative to the standard SEM-EDX protocol and had the added advantage of being much quicker.

Another interesting application was described by Taborelli et $a l .{ }^{\mathbf{1 4 9}}$ There is very little literature on the radiochemical and microscopic analysis of gunshot wounds in decomposed material and even less in skeletonized samples. There is also very little information on the survival of gunshot residues in skeletonized samples. The authors therefore undertook a study in which nine gunshot wounds were produced on pig heads and these were allowed to skeletonize for a period of four years. At the same time, four gunshot entries on human heads from judicial cases were also studied. The human samples were macerated to the bone in water prior to analysis. In all cases, analysis was performed using SEM-EDX. A partial success was reported, with four of the nine pig samples and all four of the human samples demonstrating positive results. In the human samples, two lesions showed traces of $\mathrm{Pb}$ and $\mathrm{Sb}$, one only $\mathrm{Pb}$ and the fourth $\mathrm{Ba}, \mathrm{Pb}$ and $\mathrm{Sb}$. This was a pilot study and the authors noted the necessity of further studies to ascertain the environmental modifications that occur to gunshot residue. A paper by Rinke et $a{ }^{150}$ described the use of LIBS and several chemometric techniques to discriminate between several different types of $9 \mathrm{~mm}$ ammunition. Different manufacturers and ammunition with different jackets (non-jacketed, copper jacketed and metallic alloy jacketed) were tested. Some of the bullets were fired through steel plates and the edges of the holes then analysed. Other bullets were scraped manually along steel plates and others along some porcelain. The analyses were performed using LIBS utilizing a Nd:YAG laser operating at $1064 \mathrm{~nm}$ with a pulse width of $9 \mathrm{~ns}$ and an energy of $63 \mathrm{~mJ}$ pulse $^{-1}$. Data were acquired over the wavelength range 200 to $500 \mathrm{~nm}$ with 12 
spectra being collected from each hole or scrape line. The data matrix collected was analysed using target factor analysis with a Bayesian soft classifier being used to calculate probability and the Pearson correlation coefficient also being used. Of the 36 single sample transfers, the copper-jacketed and non-jacketed ammunition had a probability of 1 of the jacket material being transferred to the porcelain after a scrape. The metal alloyjacketed ammunition was less successfully detected on both steel and porcelain substrates. The analysis of the bullet hole walls was also not entirely successful, with over $50 \%$ of the samples leaving insufficient transfer to be identified.

2.3.3. Remote analyses of inorganic materials. This section has been introduced to the review because there has been an upsurge in the number of LIBS analyses undertaken during this review period. As well as the LIBS application discussed at the end of the previous section, numerous others have been published. One of the main advantages of LIBS is that it may be used remotely, i.e. it has a "stand-off" capability that enables analytes to be measured in a substance at a distance. This means that a toxic or otherwise dangerous sample may be analysed safely. It also means that it may readily be incorporated into an automated system enabling rapid and unattended analyses.

Two papers by the same research group have determined contaminants in gases. In one, Eseller et al. ${ }^{151}$ determined He in gas mixtures comprising mainly of hydrogen. At very high concentrations of $\mathrm{He}(>7.25 \%)$ the LIBS signal was quenched because of Penning ionization. The $\mathrm{H}$ alpha line at $656.28 \mathrm{~nm}$ broadened with increasing He content. The He was determined at $587.56 \mathrm{~nm}$. The system was optimized in terms of the laser energy, the delay time between the laser pulse and the acquisition of data and the gas pressure. The signal showed good correlation with $\mathrm{He}$ concentrations up to $1 \%$ and the optimized system had a LOD of $78 \mathrm{ppm}$. In a paper by Tripathi et al. ${ }^{152}$ the work was extended to include the determination of several analytes (Ar, He, $\mathrm{N}$ and $\mathrm{O}$ ) in hydrogen fuel used for rockets. This paper provided both schematic diagrams and photographs of the system used. A frequency doubled Nd:YAG laser operating at an energy of $110 \mathrm{~mJ}$ pulse $^{-1}$ and with a pulse frequency of $10 \mathrm{~Hz}$ was focussed through the sample cell and then the emission from the analytes in the plasma was reflected back to the spectrometer which had three different gratings present. The grating that had 1200 lines $\mathrm{mm}^{-1}$ provided sufficient resolution for the determination of the analytes. The detector was an intensified CCD containing $1024 \times 256$ pixels and was operated with a $300 \mathrm{~ns}$ gate delay and a $2 \mu$ s gate width. Each recorded LIBS spectrum was the average of 450 laser pulses. Unfortunately, although the spectrometer had sufficient resolution to separate the analyte lines, it had narrow spectral range coverage and could not detect all of the analytes simultaneously. Therefore, $\mathrm{Ar}, \mathrm{N}$ and $O$ were determined (at 763.51, 746.83 and $777.35 \mathrm{~nm}$, respectively) and then the grating was moved so that the He could be determined at $587.56 \mathrm{~nm}$. A notch filter had to be used for the successful determination of $\mathrm{He}$ because otherwise, the signal was swamped by the presence of light originating from $\mathrm{H}$. The optimized system yielded LOD of 97, 25, 80 and $10 \mathrm{ppm}$ for Ar, $\mathrm{He}, \mathrm{N}$ and $\mathrm{O}$ respectively.
Double pulse LIBS has been shown in the past to offer some advantages over the single pulse version. Two papers that used double pulse LIBS are worth reporting. Heilbrunner et al. ${ }^{153}$ used double pulse LIBS to determine trace elements ( $\mathrm{Al}$ and $\mathrm{P}$ ) in iron oxide. The LIBS system used was a frequency doubled Nd:YAG laser operating with a pulse duration of $6 \mathrm{~ns}$, an inter-pulse delay time of $500 \mathrm{~ns}$ and at an energy of $140 \mathrm{~mJ}$ pulse $^{-1}$. The performances of a CCD and an intensified CCD were compared during the analysis. The operation of these different detectors including their temperature of operation $\left(-20\right.$ and $-60{ }^{\circ} \mathrm{C}$ for iCCD and CCD respectively) and their mechanisms of gating were discussed at length. Samples were placed on a rotating stage to ensure that the same spot did not undergo continual ablation. Use of the CCD detector resulted in significantly higher signal to noise ratios and consequently lower LOD for both $\mathrm{Al}$ and $\mathrm{P}$ when compared with the iCCD detector. However, the CCD detector required a greater number of laser pulses to achieve an acceptable signal quality. This would be a disadvantage when determining elements that become depleted, e.g. B. The authors presented experimental data demonstrating that an increased number of laser pulses on the same site led to a decreasing B signal whist the Fe signal remained virtually unchanged. The other paper to report the use of double pulse LIBS was provided by Suliyanti et al. ${ }^{154}$ who determined $\mathrm{Ca}$ and $\mathrm{Cl}$ in an attempt to evaluate the strength of concrete. Instead of using the same laser to provide the two pulses, as was the case in the previous paper, these authors used two separate lasers in orthogonal geometry. The first laser (Nd:YAG operating at $1064 \mathrm{~nm}$ with an energy of $110 \mathrm{~mJ}$ and a repetition rate of $10 \mathrm{~Hz}$ ) produced a plasma in the helium atmosphere about $5 \mathrm{~mm}$ in front of the sample surface. The second laser (Nd:YAG operating at $532 \mathrm{~nm}$ ) then provided a pulse $10 \mu \mathrm{s}$ after the helium plasma was formed. The ablated material then entered the helium plasma where light emitted was collected and transported via a fibre optic to the spectrometer and detector. The power of the second laser was varied. At a power of $135 \mathrm{~mJ}$, a crater with a diameter of $0.7 \mathrm{~mm}$ was created. Such a large crater is obviously undesirable. However, this was reduced in size to $10 \mu \mathrm{m}$ when an energy of $2.5 \mathrm{~mJ}$ was used. The double pulse LIBS system provided a very large enhancement in sensitivity compared with the single pulse version. The LOD for $\mathrm{Cl}$ was $80 \mathrm{ppm}$.

Darwiche et al. ${ }^{155}$ developed a LIBS method capable of determining $B$ in both solid and molten silicon. This system comprised a frequency quadrupled Nd:YAG laser $(266 \mathrm{~nm}$, operating with a $4 \mathrm{~ns}$ pulse duration, a repetition rate of $20 \mathrm{~Hz}$ and a power of $10 \mathrm{~mJ}$ pulse $^{-1}$ ), a Czerny-Turner spectrometer (with three different gratings: 1200, 2400 and 3600 grooves $\mathrm{mm}^{-1}$ ) and a $1024 \times 1024$ pixel intensified CCD detector. Ten laser shots were used to provide an emission signal that compensated for any laser beam energy fluctuations. The signal to background ratio improved when helium or argon was used instead of air at a reduced pressure (500 mbar). The signal to background ratio also improved with increasing laser energy and this was why $10 \mathrm{~mJ}$ pulse $\mathrm{s}^{-1}$ was chosen in preference to 4 $\mathrm{mJ}$ pulse $\mathrm{e}^{-1}$. Calibration was by analysis of samples with a known B concentration in silicon. A calibration graph between 
0 and $100 \mathrm{ppm}$ B was shown. Although the correlation coefficient was only 0.89 , a LOD of $0.2 \mathrm{mg} \mathrm{kg}^{-1}$ was claimed. The system was also applied to the molten silicon sample $\left(1410^{\circ} \mathrm{C}\right)$ but ensuring that the laser was moved further away (increasing the distance from $11 \mathrm{~cm}$ to $50 \mathrm{~cm}$ ) to ensure that it was not damaged by splashes of the molten material.

Analysis of zeolite materials using LIBS was reported by Hornackova et al. ${ }^{156}$ who used a Q-switched, frequency doubled $\mathrm{Nd}$ :YAG laser providing a laser pulse of $4 \mathrm{~ns}$ duration and with a maximum power of $165 \mathrm{~mJ}$ pulse $^{-1}$ to provide the plasma of excited material. Light was collected and transmitted to an Echelle type spectrometer that covered the spectral range 200 to $950 \mathrm{~nm}$ and used an intensified CCD detector. The gating time for the detector was $5 \mathrm{~ns}$. Parameters such as the time delay, gate width and laser pulse energy were all optimized and were different for different zeolite types. Similarly, the number of laser pulses averaged to provide the signals also varied with sample type, with 20 shots being sufficient for mordenite and zeolite type $\mathrm{Y}$ but 50 shots being required for ZSM-5. Molar ratios of $\mathrm{Si}$ : $\mathrm{Al}$ for the calibration were determined using a traditional wet chemical technique and were in the range of 5.3 to 51.8 for mordenite, 2.3 to 12.8 for zeolite type Y and 14 to 600 for ZSM-5. Correlation coefficients were typically greater than 0.97 .

A traditional LIBS method using fixed energy laser pulses was shown to be incapable of accurately determining $T i$ in aluminium oxide ${ }^{157}$ This was attributed to matrix effects causing the plasma temperature to differ significantly with differing amounts of Ti present. A calibration curve with a correlation coefficient of only 0.9457 was obtained. Zhang et al., therefore, developed a method by which calibration was achieved using a fixed temperature plasma. The temperature of the plasma was, however, affected by the depth of the crater produced in the sample. To overcome this problem, the sample was moved continuously, i.e. each laser pulse ablated a fresh part of the surface. The energy of the laser pulse was also varied with Ti content to ensure that the same plasma temperature was maintained. The resulting correlation coefficient was increased to 0.9938 . Precision was also improved from $12.5 \%$ RSD with fixed energy calibration to $4.3 \%$ RSD with fixed plasma temperature calibration. It was unclear how the analysis of a sample could be achieved when there was no prior knowledge of its Ti content.

Several other more simple applications of LIBS were provided. These included the determination of numerous analytes in fertilizer, ${ }^{158}$ the analysis of different brands of talcum powder for their $\mathrm{Cr}$ and $\mathrm{Pb}$ content ${ }^{159}$ and the determination of $\mathrm{CaCO}_{3}$ and $\mathrm{SiO}_{2}$ content in historic lime mortars. ${ }^{160}$ In the paper by Gondal et al. ${ }^{159}$ the LOD obtained were 1.72 and $1.96 \mathrm{ppm}$ for $\mathrm{Cr}$ and $\mathrm{Pb}$ respectively. The levels found in the talcum powders ranged from 20 to $30 \mathrm{ppm}$ for $\mathrm{Cr}$ and from 15 to $20 \mathrm{ppm}$ for $\mathrm{Pb}$.

2.3.4. Other applications of the analysis of inorganic materials. Two papers have described the preparation of isotopically enriched reference materials. Novotnik et al. ${ }^{161}$ described the preparation of ${ }^{50} \mathrm{Cr}(\mathrm{VI})$ and ${ }^{53} \mathrm{Cr}(\mathrm{III})$ from the enriched oxides without the use of either oxidizing or reducing agents. $\operatorname{The} \mathrm{Cr}(\mathrm{vI})$ solution was prepared by fusion of ${ }^{50} \mathrm{Cr}$ enriched chromium oxide with sodium potassium carbonate and sodium hydroxide in a platinum crucible on a Bunsen burner. After cooling, the melt was dissolved in concentrated $\mathrm{HCl}$ and then diluted to $10 \mathrm{~mL}$. The $\mathrm{Cr}$ (III) was prepared by microwave assisted nitric acid dissolution of ${ }^{53} \mathrm{Cr}$ enriched chromium oxide. After dissolution, the material was transferred to a platinum crucible, evaporated to $0.2 \mathrm{~mL}$ and then $\mathrm{HCl}$ added before dilution to $10 \mathrm{~mL}$. The concentrations of the materials were determined using ID-ICP-MS and were $197.2 \pm 0.8 \mathrm{mg} \mathrm{L}^{-1}$ and $200.3 \pm$ $0.9 \mathrm{mg} \mathrm{L}^{-1}$ for ${ }^{50} \mathrm{Cr}(\mathrm{III})$ and ${ }^{53} \mathrm{Cr}(\mathrm{VI})$ respectively. The purity of the compounds was verified by speciation analysis using the coupling of an anion exchange column with ICP-MS detection. The chromatographic conditions were discussed in the paper. The speciation system was also used to determine the effects of trace oxidants or reductants in the samples. In the absence of any oxidants or reductants, the species were stable for at least 18 months. The other paper to report the production of isotopic reference materials was by Vogl and Rosner. ${ }^{162}$ These workers produced and then certified a unique set of isotope reference materials (ERM-AE102a and ERM-AE104a) in which the absolute isotope ratios were determined and offset $\delta{ }^{11} \mathrm{~B}$ materials (ERM-AE120, ERM-AE121 and ERM-AE122) for B isotope determination in sample types as diverse as geochemical, environmental and industrial. All of the materials were prepared by mixing (under full gravimetric control) parent solutions enriched in ${ }^{10} \mathrm{~B}$ or ${ }^{11} \mathrm{~B}$ with a parent solution of natural $\mathrm{B}$ isotopic content. The $\mathrm{B}$ isotopic composition in the parent solutions was determined using TIMS. The resulting solutions were analysed to determine total B content as well as their isotopic composition using ID-TIMS. For all five reference materials the isotopic composition obtained on the basis of gravimetric data agreed well with those obtained using the TIMS techniques. Stability and homogeneity tests indicated that there was no effect on isotopic composition or on the related uncertainties. The certified $\delta^{11} \mathrm{~B}$ values were: $-20.2,19.9$ and 39.7 parts per thousand for ERM-AE120, -121 and -122, respectively, meaning that they were the first certified materials with $\delta^{11} \mathrm{~B}$ values that were not zero parts per thousand. For ERMAE102a and ERM-AE104a, the certified ${ }^{10} \mathrm{~B}$ isotope abundances were 0.29995 and 0.31488 respectively with expanded uncertainties of 0.00027 and 0.00028 .

Other papers have also reported isotopic analysis of materials, but have not produced reference materials. Gautier et al. ${ }^{163}$ used TIMS to undertake the isotopic analysis of germanium. A new sample loading protocol was developed in which germanium powder was mixed with a commercial cellulose nitrate adhesive that had been diluted in acetone. The mixture (totalling approximately $0.3 \mathrm{mg}$ of $\mathrm{Ge}$ ) was then placed on the rhenium filament. The advantage of the technique was that it avoided the problems associated with the dissolution of the germanium (neither a mixture of $\mathrm{HNO}_{3}$ and $\mathrm{H}_{2} \mathrm{O}_{2}$ nor aqua regia dissolved the sample completely). Unfortunately, there is no suitable CRM for Ge isotopic analysis and so the authors used some natural germanium under the assumption that it would have the natural isotopic composition published by IUPAC to enable them to calculate the mass fractionation factor which was then applied to the analysis of two enriched samples (containing 84.10 and $82.60 \%$ of ${ }^{76} \mathrm{Ge}$ ). The only other isotope of note was 
${ }^{74} \mathrm{Ge}$, which was present at approximately $14 \%$. The other isotopes were all $<1 \%$ abundant. Determination of the ${ }^{76} \mathrm{Ge} /{ }^{74} \mathrm{Ge}$ isotope ratio led to a precision of between 0.2 and $0.3 \%$ being obtained $(n=6)$. Huyskens et al. ${ }^{164}$ evaluated different sources of colloidal silica gels as emission activators for ionization of $\mathrm{Pb}$ during TIMS analyses. As well as the $\mathrm{Pb}$ and $\mathrm{U}$ blank levels in the materials, the authors also tested their ionization efficiency, signal stability, degree of fractionation and the reproducibility and accuracy of the $\mathrm{Pb}$ isotopic ratios measured using them as activators. Further tests to evaluate the dependency of the ionization on particle size, the concentration of the silica gel and the mixing ratio of the gels with phosphoric acid were also performed. Mass fractionation, reproducibility, and stability of the ion beam were monitored using the $\mathrm{Pb}$ isotope standard SRM 981. The products from four manufacturers were compared. Two provided far better isotope ratio data than the other two whilst also having significantly less $\mathrm{Pb}$ and $U$ contamination. As well as providing more accurate ratios, they were also precise with precision for the ${ }^{207} \mathrm{~Pb} /{ }^{206} \mathrm{~Pb}$ ratio being $0.05 \%$. Ionization efficiency was not dependent on the particle size but did depend on the concentration of the silica gel and its mixing ratio with phosphoric acid. The highest ionization efficiency $(9.2 \pm 2.2 \%)$ was obtained using $0.004 \mathrm{~mL}$ of silica gel at a concentration of $0.4 \%$ by weight of silica loaded with $0.05 \mathrm{~mL}$ of $0.02 \mathrm{~N}$ phosphoric acid.

Numerous interesting applications have been published in this review period, although there is no trend towards any particular topic area. Heinrich and Kipphardt ${ }^{\mathbf{1 6 5}}$ used methane as a gaseous modifier during the ETAAS determination of Si in aqueous samples. Using a mixture of 5\% methane in argon that was bled into the atmosphere of the ETAAS tube, significant improvements in peak shape (a double peak in the absence of the methane being transformed into a single peak) and sensitivity (a two-fold increase) were obtained. In addition, increased pyrolysis temperatures were achievable. These improvements occurred at pyrolysis temperatures greater than $1300{ }^{\circ} \mathrm{C}$ and this was attributed to the methane decomposing, producing a very reducing atmosphere as well as soot. The production of soot caused a significant increase in the background signal during atomization. Consequently, a flush period of 6 to $10 \mathrm{~s}$ duration, where only argon was introduced, was inserted between the pyrolysis and atomization stages. This removed much of the soot leading to improved atomic absorption whilst still obtaining the benefits provided by the reducing atmosphere. Under optimal conditions (pyrolysis and atomization temperatures of 1350 and $2450{ }^{\circ} \mathrm{C}$, respectively), characteristic masses (i.e., the mass of the analyte giving rise to an absorbance of 0.0044 ) of 50 and $30 \mathrm{pg}$ were obtained using a standard transversely heated graphite atomizer and an end-capped transversely heated graphite atomizer. These were less than other matrix modifiers that had been used previously. An added advantage of the gaseous modifier was that it introduced fewer contaminants than liquid modifiers. This enabled a very low LOD of $0.2 \mu \mathrm{g} \mathrm{L}^{-1}$ to be obtained.

A paper by Resano et al. ${ }^{\mathbf{1 6 6}}$ described the analysis of silica using solid sampling ETAAS. The procedure for the solid sampling was slightly different to the usual protocols in that carbon $(1.5 \mathrm{mg})$ was first weighed onto the platform, followed by the sample and then the liquid modifiers palladium and nitric acid (if required). The platform was then introduced to the instrument in the normal way. Calibration was achieved using aqueous standards. The method was validated by using the CRM NCS DC73304. Although this is a rock sample, it comprises $90 \% \mathrm{Si}$, with $\mathrm{Al}$ and $\mathrm{Fe}$ both representing approximately $3 \% \mathrm{~m} \mathrm{~m}^{-1}$ and was therefore of a similar composition to the materials being analysed. The temperature programs used were optimized for each of the analytes (As, Cd, Cr, Cu, Pb and $\mathrm{Sb}$ ). In the absence of the graphite powder, a hole was quickly formed in the platform and this was attributed to the reaction between the silica and the carbon. Addition of the graphite powder extended the lifetime of the platform significantly. As well as the use of the CRM, the data obtained using solid sampling ETAAS were compared with those obtained using a dissolution followed by ICP-OES determination. The results were in agreement although the ETAAS method provided significantly better LOD $\left(<0.1 \mathrm{mg} \mathrm{g}^{-1}\right.$ for all analytes). Precision was $6-9 \%$ which was fit for purpose and is normal for solid sampling ETAAS.

Rey-Raap and Gallardo ${ }^{167}$ used $C V$-AAS to determine $\mathrm{Hg}$ distribution in spent compact fluorescent lamps. Three phases of the lamps were analysed: the vapour phase, the glass and the phosphor powder. Median values were $24.52 \pm 0.3 \mathrm{ppb}$, $204.16 \pm 8.9 \mathrm{ppb}$ and $18.74 \pm 0.5 \mathrm{ppb}$ for the vapour, the phosphor and the glass respectively. The overall concentration depended on the manufacturer and on the year of manufacture. However, in all cases, the large majority of the $\mathrm{Hg}$ congregated in the phosphor powder $(\sim 85.76 \%)$ and a smaller amount diffused though the glass matrix (13.66\%). If all of the powder is removed from the glass by washing, the glass may be considered to be non-hazardous waste.

A separation-enrichment technique was described by Soylak and Murat ${ }^{\mathbf{1 6 8}}$ who determined $\mathrm{Co}, \mathrm{Cu}, \mathrm{Fe}$ and $\mathrm{Pb}$ in table salts. The salt was dissolved in water, buffered to $\mathrm{pH} 6$ and then the analytes were chelated with violuric acid and the chelates then retained on multi-walled carbon nanotubes. After removal of the solution, the analytes could be eluted and then determined using FAAS. Parameters such as $\mathrm{pH}$, amount of violuric acid, flow rates, eluent type and sample volume were all investigated. Under optimized conditions, the quantification limits were $0.36 \mu \mathrm{g} \mathrm{g}^{-1}, 0.43 \mu \mathrm{g} \mathrm{g}^{-1}, 0.15 \mu \mathrm{g} \mathrm{g}^{-1}$ and $0.38 \mu \mathrm{g} \mathrm{g}^{-1}$ for $\mathrm{Cu}, \mathrm{Pb}$, Co and Fe, respectively and precision was better than 10\% RSD. The protocol was validated by the analysis of several CRMs, including TMDA 54.4 fortified lake water, NIST 1515 apple leaves and HR-1 Humber river sediment. The analysis of the salt was partially successful, with Fe being found in the concentration range 1.6 to $6.4 \mu \mathrm{g} \mathrm{g}^{-1}$ and $\mathrm{Pb}$ being found in only one sample, but at a concentration of $5.0 \mu \mathrm{g} \mathrm{g}^{-1}$. The other analytes were still less than the LOD, despite the preconcentration factor.

A novel application was described by Bucker and Acker ${ }^{\mathbf{1 6 9}}$ who used high resolution continuum source absorption spectroscopy to determine $\mathrm{HNO}_{3}, \mathrm{HF}$ and $\mathrm{H}_{2} \mathrm{SiF}_{6}$ in process etching solutions used to treat the saw damage inflicted on silicon wafers. Since the solar cells need to have a consistent 
texturization, the composition of the etching baths needs to be known and maintained at a constant level. The Si was monitored at the $251.611 \mathrm{~nm}$ line whereas the nitrate was measured as the molecular absorption arising from NO at a wavelength of $214.803 \mathrm{~nm}$ and the fluoride was monitored through the AlF molecule at $227.46 \mathrm{~nm}$. The characteristic masses were actually quite impressive, with that for nitrate being the worst at $20 \mathrm{ng}$, the Si was $130 \mathrm{pg}$ and the fluoride was $13 \mathrm{pg}$. Therefore, huge dilutions of the sample were required for them to come onto the linear range. Calibration was against aqueous standards. A lanthanum modifier was used for the electrothermal molecular absorbance of the fluoride and of the nitrate and a palladiummagnesium nitrate modifier was used for the determination of the Si. Alternative techniques were used for method verification, with ICP-OES determining the Si and ion chromatography determining the nitrate and hydrofluoric acid. In general, the results obtained using the alternative methods were in good agreement with those data obtained using the proposed methodology. The relative uncertainties were greater for the developed method when compared with the alternative, but the levels were still fit for purpose. The nitrate and $\mathrm{Si}$ could be determined directly. However, the hydrofluoric acid had to be calculated as the difference between total fluoride and that present within the molecule $\mathrm{H}_{2} \mathrm{SiF}_{6}$. Spiking experiments led to recoveries within the range $96-105 \%$ for most analytes in most of the samples measured. The authors concluded that their method had the advantage of being capable of determining the acids and the metal impurities using the same instrument.

Castell et al. ${ }^{170}$ developed a TXRF and ICP-OES method capable of analysing liquid phase laser-ablated nanoparticles of cryolite-alumina solutions originating from industrial HallHerault reduction cells. The resulting technique was capable of determining the $\mathrm{Al}: \mathrm{Na}$ ratios with adequate precision for aluminium production plant cell diagnostics whilst also being able to identify trace contaminant elements. Molten samples were taken directly from the production plant and placed under ultra-pure water in a quartz cell. From here they were laser ablated using the third harmonic of a Nd:YAG laser and the water suspension deposited on quartz reflectors for TXRF analysis or aspirated directly into the nebuliser of an ICP-OES instrument, with calibration using aqueous standards. The results obtained using the developed methods were compared with those obtained using a dissolution process of the cryolite followed by standard methods of quantification.

Numerous analytical techniques were employed by Bencs et $a{ }^{171}$ to determine the $\mathrm{Cr}, \mathrm{Fe}$ and $\mathrm{Mn}$ dopants in lithium niobate optical crystals. The samples were analysed using solidsampling ETAAS and solution-based analyses such as ETAAS, FAAS, ICP-OES and ICP-MS. The crystals were first pulverized to form a homogeneous powder. For the solid-sampling ETAAS protocol, the powders were weighed $(0.05$ to $5 \mathrm{mg}$, depending on the analyte) directly onto platforms ready for insertion into the tube. Standard additions were required to obtain reliable data, with the analyte addition from aqueous standards preserved in $0.05 \mathrm{M} \mathrm{HNO}_{3}$ or $0.24 \mathrm{M} \mathrm{HCl}$. The addition of $20 \mu \mathrm{L}$ of the standards and the drying process occurred prior to weighing of the sample. The dissolution process involved the material being heated in a platinum crucible with potassium chloride and sulfuric acid. After cooling, the solid melt was dissolved in either $8 \mathrm{M} \mathrm{HF}$ or $1 \mathrm{M}$ triammonium citrate before being diluted to $100 \mathrm{~mL}$. Boric acid was then introduced to remove the fluoride prior to analysis using ICP-OES. The sample had to be diluted further for ICP-MS measurements, but remained undiluted for FAAS and ICP-OES determinations. Each of the protocols was optimized and the resulting figures of merit presented in tabular form. Unsurprisingly, the solutionbased ICP-MS technique provided the best LOD and the FAAS supplied the worst. The solid sampling ETAAS method provided quite poor LOD, but this was attributed to the necessity of using less sensitive lines to accommodate the high concentrations present in the crystal and the additions made for calibration. In addition, an increased internal argon flow rate was also required to decrease sensitivity. The authors rounded off their study by using XANES to study the residue left on the platform used in ETAAS.

2.3.5. Analysis of nano-structures. The use of nano-materials is still gaining significant interest because they have very diverse applications including drug delivery, bactericides, catalysts, electronic components etc. This review is designed to summarise advances in the atomic spectrometric analysis of such materials rather than give a general overview of their uses. The review is, therefore, somewhat selective.

Several reviews and overviews of the analysis of nano-materials have been published during this review period. A publication by Baer ${ }^{172}$ briefly discussed ISO technical report 14187 , which described the use of surface analysis tools (SIMS, XPS, RBS, Auger electron spectroscopy, scanning probe microscopy, $\mathrm{X}$-ray reflectometry (XRR), low energy ion scattering (LEIS), TEM and AFM). Bauer's paper did not discuss any of the techniques in detail, but it did give an assortment of other ISO technical reports that may be of relevance and briefly mentioned some of the potential problems associated with the analysis of nanoparticles, e.g., the effect of particle shape, damage done to the particle using some techniques, etc. Any reader interested in the subject would probably be better off referring to the original ISO report.

A lengthy review (with 232 references) by Senoner and Unger ${ }^{173}$ entitled "SIMS imaging of the nanoworld: applications in science and technology" discussed the state of the art in nano-scale SIMS analysis. A brief introduction in which SIMS and other spectrometric methods are compared was followed by a slightly more substantial section in which recent instrumentation for high resolution SIMS imaging and the limiting factors of lateral resolution were discussed. The bulk of the review gave examples from a number of different sample types including the analysis of the nanoparticles themselves, as well as environmental, geological, cosmological, materials science, semiconductors, nano-scaled polymer fibers, medical research, life science and surface analysis applications. The review finished off with a short section that gave a brief summary of the future challenges facing SIMS analysis of such materials.

Krystek $^{\mathbf{1 7 4}}$ reviewed (with 54 references) approaches to biodistribution studies for engineered gold and silver nanoparticles. An overview of the recent applications of gold and silver 
nanoparticles was also provided, but the main thrust of the review was their determination using ICP-MS.

2.3.5.1. Asymmetric flow field flow fractionation. Asymmetric flow field flow fractionation (A4F) is a well known method for determining the particle size and distribution of nano-sized particles, and is coming increasingly applied to the analysis of engineered nanoparticles. It has been known for many years that many nanoparticle types can be problematic in that they readily agglomerate, forming far larger assemblies of particles. These cause some serious problems for analysts in that they readily sink to the bottom of containers, providing inhomogeneous samples. Even if they are kept in suspension, the agglomerates are often so large that they will be discriminated against by the nebuliser-spray chamber assemblies of ICP instruments and will not reach the plasma. Significant underestimates of the nanoparticle concentration can, therefore, be obtained. Many nanoparticle materials are stabilized using dispersants or coatings. However, both of these are prone to failure if there is a change in ionic strength of the sample (e.g. if it is changed by dilution), change in $\mathrm{pH}$ etc. A paper by Ulrich et $a .^{175}$ discussed the critical aspects of sample handling for direct nanoparticle analysis and the analytical challenges to using A4F coupled online with numerous detector types. The paper by Ulrich et al. adopted a multi-detector approach in which the A4F separation was coupled online with a diode array UV-Vis detector, a multi-angle laser light scattering (MALLS) instrument in which a glass fibre optic collected light at $108^{\circ}$ and presented that to a dynamic light scattering (DLS) detector and an ICP-MS instrument. The flow through the first two detectors was split by a factor of 200 for the ICP-MS analysis and an internal standard $\left(10 \mu \mathrm{g} \mathrm{\textrm {L } ^ { - 1 }}\right.$ Ir) was added online to help overcome non-spectroscopic interferences. Several types of nanoparticles were investigated including silver coated with citric acid, silver coated with polyvinylalcohol and titanium oxide functionalized with polyacrylate. Both types of silver nanoparticle increased in size when diluted (from 10-12 nm to $>25 \mathrm{~nm}$ ) whereas the titanium nanoparticles remained unchanged. Addition of sodium nitrate had a similar, but more significant, effect on the silver nanoparticles. A change of $\mathrm{pH}$ delivered significant changes to the nanoparticles. The silverpolyvinylalcohol ones agglomerated rapidly and reached $150 \mathrm{~nm}$ over a period of 120 minutes. The silver-citrate ones increased to $120 \mathrm{~nm}$ over 60 minutes and then stabilized. The titanium-polyacrylate particles increased from approximately $300 \mathrm{~nm}$ to $2000 \mathrm{~nm}$ over a time period of 60 minutes. Another problem noted in the study was that of particle-membrane interactions; caused by electrostatic charges. Three membrane materials were tested: polyethersulfone, regenerated cellulose and polyvinylidene difluoride. The authors proposed a hypothesis that could be used to explain the interactions, but admitted that further study was required to substantiate it.

A similar paper by some of the same authors ${ }^{176}$ compared their multi-detector approach with TEM and batch DLS detection. In addition to characterizing different silver nanoparticle types, the authors also distinguished between nanoparticulate and ionic Ag by use of an ultracentrifugation protocol. The multidetector approach led to greatly improved reliability of data compared with those obtained using batch DLS detection and was significantly faster than TEM whilst also enabling the materials to be determined directly in suspension. Loeschner et $a$ l. $^{177}$ also developed a multi-detector system for the determination of polyvinylpyrrolidone-stabilized silver nanoparticles. Again, A4F was coupled on line with light absorbance, light scattering and ICP-MS detection systems with each of the components giving slightly different information on the nanoparticulate materials being eluted. Optimization of the $\mathrm{A} 4 \mathrm{~F}$ conditions demonstrated that carrier liquid composition, membrane material, cross flow rate and spacer height had a significant effect on the recoveries and the retention times; i.e. their findings were broadly in agreement with those found by Ulrich et al. above. Size calibration was obtained using two approaches, namely the use of polystyrene nanoparticles and from theory. These authors also used fraction collection followed by TEM analysis to confirm the particle size distribution and to provide information on the particle shape.

A further paper that used $A 4 F$ coupled to numerous detectors was prepared by Gigault and Hackley. ${ }^{178}$ Again A4F was coupled with UV-Vis, light scattering (static and dynamic) detectors and an ICP-MS instrument. Using this combination of detectors, it was possible to determine the mass isotopic signature of the silver nanoparticles as a function of their size and optical properties. This provided the specificity that is necessary for differentiating labelled silver nanoparticles from their naturally occurring or anthropogenic analogues. The authors applied their methodology to the detection of silver nanoparticles in estuarine sediments. A sediment suspension was doped with enriched ${ }^{109} \mathrm{Ag}$ nanoparticles stabilized using humic and fulvic acids. The data obtained indicated that the nanoparticles interacted not only with different particulate components of the sediment but also with themselves, forming agglomerates. Such a protocol should enable elucidation of the biological and environmental fate of silver nanoparticles.

An online A4F-ICP-MS approach for the detection of silver nanoparticles in wastewater was developed by Hoque et al. ${ }^{\mathbf{1 7 9}}$ The particulates could be quantified in terms of both size and concentration. Both standard addition and external calibration approaches were tested for the determination of the concentration and gave similar results. Using nanoparticulates of 20 , 40 and $60 \mathrm{~nm}$ for size calibration, a clearly resolved fractogram was obtained with the retention time of the nanoparticulates clearly correlating with the particle size. The method was applied to the analysis of wastewater where a concentration of $1.9 \mathrm{ng} \mathrm{mL}^{-1} \mathrm{Ag}$ was found. The modal particle size of the $\mathrm{Ag}$ present in the wastewater was $9.3 \mathrm{~nm}$. The procedure provided a LOD of $0.8 \mathrm{ng} \mathrm{mL}{ }^{-1}$.

An interesting paper by Gray et al. ${ }^{\mathbf{1 8 0}}$ compared hydrodynamic chromatography and A4F, both coupled with ICP-MS detection. The hydrodynamic chromatography system used was a PL-PSDA column, that was capable of separating particles between 5 and $300 \mathrm{~nm}$ diameter. The mobile phase was $0.5 \mathrm{M}$ disodium hydrogen phosphate, $0.05 \%$ Triton X-100, 0.05\% formaldehyde and $0.013 \%$ sodium dodcecyl sulfate, adjusted to $\mathrm{pH}$ 7.5. The optimal flow rate was $1.7 \mathrm{~mL} \mathrm{~min}^{-1}$, but a flow splitter postcolumn decreased the rate of flow to the ICP-MS instrument by 
approximately 55\%. Strangely, the A4F conditions were not optimized. Instead, the authors used literature recommendations. Using gold nanoparticles of diameter 5, 20, 50 and $100 \mathrm{~nm}$ as the analyte, A4F was shown to have significantly better resolution, with the differences being especially pronounced at the smaller size range. Hydrodynamic chromatography did, however, present some advantages. Recoveries of the analyte particles ranged from 77 to $96 \%$, which was somewhat better than those for $\mathrm{A} 4 \mathrm{~F}$, which ranged from 4 to $89 \%$. For $\mathrm{A} 4 \mathrm{~F}$, the poor recovery was especially prevalent for the larger particles. It is possible that, had the A4F conditions been optimized, recovery values could have improved. The hydrodynamic chromatography could also distinguish between dissolved, ionic gold and nanoparticulate gold, which the A4F could not. The LOD for both techniques was

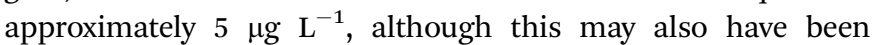
improved had rigorous optimization been undertaken.

An improved sample preparation method for the determination of titanium dioxide nanoparticles in sunscreens was reported by Nischwitz and Goenaga-Infante. ${ }^{181}$ The sunscreens $(0.1 \mathrm{~g})$ were first de-fatted using hexane $(10 \mathrm{~mL})$ and the hexane phase separated using centrifugation and then removed completely using a Pasteur pipette. The residue was then sonicated in the presence of an aqueous extractant $(10 \mathrm{~mL})$. This provided a stable suspension of nanoparticles. Addition of a small amount of hexane $(10 \mathrm{~mL})$ and mixing led to any agglomerated particles disaggregating. After leaving for an hour for the phases to separate, the aqueous suspension was collected ready for characterization using A4F-ICP-MS. The A4F conditions were: regenerated cellulose membrane, a spacer of $350 \mu \mathrm{m}$ and a carrier of water. The cross flow programme used was also cited in the paper. Particle size calibration was achieved using gold particles of 10, 30 and $60 \mathrm{~nm}$ diameter. The eluate from the $\mathrm{A} 4 \mathrm{~F}$ was diluted online with $3 \%$ nitric acid containing the internal standards $\mathrm{Rh}$ and Y. This diluent was replaced during blank runs with standards of known Ti content. This enabled calibration of the concentration to be achieved. The protocol developed was applied to an assortment of sunscreen products that possessed different protection factors. The Ti extraction efficiency, particle size distribution and titanium dioxide recovery from the A4F system were determined for each product.

Mitrano et al. ${ }^{\mathbf{1 8 2}}$ used two of the most powerful methods available for the analysis of engineered nanoparticles, comparing single particle characterization and $\mathrm{A} 4 \mathrm{~F}$ for the analysis of silver nanoparticles. This paper will be discussed at length in the next section.

Numerous other techniques have been applied to the analysis of nanoparticulate materials. Many of these are "bulk analysis" techniques, in which the presence of the nanoparticulate is determined by the overall concentration present in a material, be it a biological material, environmental sample etc. Numerous such examples exist but they do not really increase the knowledge of atomic spectrometry. They mainly pose the challenge of representative sampling, i.e., ensuring that the nanoparticles are either dissolved completely along with the matrix by using an appropriate acid (sonicated or vortex mixed prior to sample introduction), dispersed using materials such as Triton X-100, or any other sample preparation procedure which will ensure that agglomeration does not occur. As such, the large majority of these papers will not be discussed in this review. Instead, those techniques that are less commonly used, those that may be used online to monitor the production of nanoparticles or, in some other way, deliver new information will be concentrated upon. The first of these techniques to be discussed is SIMS. This is a technique capable of analysing very small areas and also of depth-profiling. However, its use for the analysis of nanomaterials has been limited. Chia et al. ${ }^{183}$ used TOF-SIMS on vertically standing nanowires embedded in Cyclotene polymer. Embedding the nanowires in the polymer enabled the top surface of the sample to be made planar whilst also protecting the background substrate from the primary ion beam. By analysing successively lower levels, the elemental profile of the nanowire as a function of length could be determined. Calibration was achieved using thin film standards. The paper reportedly demonstrated the first use of conventional SIMS to determine the doping density with excellent depth resolution and analytical accuracy for nanowire-based materials. A paper by Ling et al. ${ }^{184}$ used the primary ions $\mathrm{C}_{60}{ }^{2+}$ (50 keV) and $\mathrm{Au}_{400}{ }^{4+}(520 \mathrm{keV})$ to bombard a series of gold nanoparticles of different sizes $(2,5,10,15,20$ and $450 \mathrm{~nm}$ in diameter). Emissions of $\mathrm{Au}^{2-}$ and $\mathrm{Au}^{3-}$ were dependent upon particle size and this was attributed to different mechanisms of energy transfer and energy densities deposited into the nanoparticles. The optimal particle size and primary ion beam for the production of $\mathrm{Au}^{2-}$ and $\mathrm{Au}^{3-}$ ions were: $20 \mathrm{~nm}$ using $\mathrm{Au}_{400}{ }^{4+}$ and $10 \mathrm{~nm}$ using $\mathrm{C}_{60}{ }^{2+}$, respectively.

The technique of LIBS has been used by Zhang et al. ${ }^{185}$ for the analysis of titanium dioxide nanoparticle aerosols during flame synthesis. Titanium tetraisopropoxide was fed into a stagnation swirl flame setup (the design and operating conditions of which are given in the paper) and this resulted in the production of the nanoparticles. A novel low energy LIBS system used a laser beam operating at $532 \mathrm{~nm}$ with a frequency of $10 \mathrm{~Hz}$ and an energy of $35 \mathrm{~mJ}$ per pulse $\left(28 \mathrm{~J} \mathrm{~cm}^{-2}\right)$. The laser beam caused an area of approximately $200 \mu \mathrm{m}$ in diameter and $1 \mathrm{~mm}$ in length to emit light and this was collected at $90^{\circ}$ using a $0.5 \mathrm{~m}$ spectrometer containing a grating (2400 grooves per $\mathrm{mm}$ ) and an intensified CCD camera. The collection optics and detection system were described in detail. At the energy used, a macroscopic spark was not produced and the system was capable of generating emission from the particles, but not from the precursor compound. The emission intensity increased with increasing particle size, but then plateaued when the particle was greater than six $\mathrm{nm}$ in diameter. However, when the precursor concentration is above $150 \mathrm{ppm}$ (corresponding to a particle size of 6 to $8 \mathrm{~nm}$ ) the emission intensity increases linearly with concentration. According to the authors, the system developed could be useful for tracking nanoparticle formation and for measuring particle volume fraction during gas-phase synthesis. In addition, the size-dependent absorption efficiency of the particles could be used to determine the particle size in situ. A second LIBS system was described by Diwakar et al. ${ }^{\mathbf{1 8 6}}$ This paper also provided a brief review of recent research that covered both fundamentals and applications. The experimental work reported the use of aerosol particle Poisson 
sampling statistics and the analysis of laser ablation particles via aerosol LIBS to minimize matrix effects for the analysis of bulk solids. Also reported was a novel micro-needle particle concentration scheme that was capable of near real-time analysis. This latter part used a pair of micro-needles in the flow path of the particles. The particles were electrostatically charged using a corona field generated between the two needles by applying a high voltage across them. The particles were deposited on the tip of one of the $500 \mu \mathrm{m}$ diameter needles (the tip was the only exposed part since the walls were insulated). The laser beam from a low energy ( $20 \mathrm{~mJ}) \mathrm{Nd}$ :YAG system could then be focussed on the end of the needle. The system was applied to the analysis of copper nanoparticles.

Two papers described the determination of gold nanoparticles in environmental samples using cloud point extraction. In the first of these, by Hartmann and Schuster, ${ }^{\mathbf{1 8 7}}$ sample $(40 \mathrm{~mL})$ was vortex mixed with $800 \mu \mathrm{L}$ of $10 \%$ Triton $\mathrm{X}-114$, $400 \mu \mathrm{L}$ of $1 \mathrm{M}$ sodium thiosulfate, $400 \mu \mathrm{L}$ of $1 \%$ citric acid and $400 \mu \mathrm{L}$ of $0.1 \mathrm{M}$ hydrochloric acid and then heated to $40{ }^{\circ} \mathrm{C}$ to induce cloud formation. The phases were allowed to separate by either placing the sample in an oven or by centrifugation at 3500 rpm for $15 \mathrm{~min}$. Placing the sample tube in ice thickened the surfactant rich phase and allowed the aqueous phase to be removed more easily. Aliquots $(20 \mu \mathrm{L})$ of the surfactant rich phase were then analysed using ETAAS. Initially, approximately $14 \%$ of the $\mathrm{Au}^{\mathrm{III}}$ was extracted into the surfactant phase, hence potentially interfering with the signal obtained from ionic and particulate Au species. The presence of the thiosulfate reduced all $\mathrm{Au}^{\mathrm{III}}$ ions to $\mathrm{Au}^{\mathrm{I}}$ and then formed a negatively charged complex with them, preventing their extraction into the surfactant phase. The extraction efficiency of the gold nanoparticles was dependent on the size, but ranged from $101 \pm 6 \%$ for particles of $2 \mathrm{~nm}$ diameter down to $52 \pm 16 \%$ for those up to $150 \mathrm{~nm}$. The presence of organic material in the samples was tolerable to a concentration of $10 \mathrm{mg} \mathrm{L}^{-1}$. The proposed method yielded a preconcentration factor of 80 and therefore provided a very low LOD $\left(5 \mathrm{ng} \mathrm{L}^{-1}\right)$. Precision $(n=12)$ at a concentration of $100 \mathrm{ng} \mathrm{\textrm {L } ^ { - 1 }}$ was $9.5 \% \mathrm{RSD}$ and spiking experiments returned recoveries of better than $91 \%$. A second paper that used cloud point extraction to preconcentrate nanoparticles from water samples was prepared by Majedi et al. ${ }^{188}$ These authors used a three level orthogonal array design to optimize the surfactant (Triton X-114) concentration, the $\mathrm{pH}$, ionic strength, incubation temperature and incubation time during the extraction of zinc oxide nanoparticles. The contribution of the individual components to the extraction efficiency was then evaluated using ANOVA. Optimal extraction efficiency still was not quantitative, but achieved $87.3 \%$. This was obtained using $0.25 \%$ Triton $\mathrm{X}-114, \mathrm{pH}=10$, salt content of $15 \mathrm{mM}$ sodium chloride, an incubation temperature of $45{ }^{\circ} \mathrm{C}$ and a time of $30 \mathrm{~min}$. Preconcentration factors of 62 and 220 were obtained for $0.25 \%$ and $0.05 \%$ Triton $\mathrm{X}-114$ respectively. Inspection of the nanoparticulates using TEM before and after extraction indicated that the particulates did not change in shape or size during the extraction process. After extraction, the zinc oxide nanoparticles were digested using microwave digestion and analysed using ICP-MS. A LOD of $0.05 \mu \mathrm{g} \mathrm{L^{-1 }}$ was obtained.
Soto-Alvaredo et al. ${ }^{189}$ developed an analytical system that coupled reversed phase liquid chromatography with ICP-MS detection to speciate silver nanoparticles and Ag ions. Addition of thiosulfate to the eluent enabled the elution of $\mathrm{Ag}^{\mathrm{I}}$ from the column without degradation of the nanoparticle species. Recovery was $>80 \%$ for both species and detection limits were $<1 \mu \mathrm{g} \mathrm{\textrm {L } ^ { - 1 }}$. The use of gold nanoparticles as an internal size standard was recommended. The methodology was applied to several sample types including foetal bovine albumin (although this had an effect on the chromatography) and textiles. Included in these was the determination of silver species in sports socks. Both nanoparticulate and ionic Ag were observed in the extracts. Despite the advances made, the authors admitted that further work was required for quantitative and reliable data to be achieved.

Jiang et al. ${ }^{\mathbf{1 9 0}}$ determined $\mathrm{Au}$ and $\mathrm{S}$ in acid digests of ${ }_{\text {-cysteine }}$ modified gold nanoparticles. Polyatomic interferences arising from $\mathrm{O}_{2}$ on the ${ }^{32} \mathrm{~S}$ isotope during ICP-MS analysis were circumvented by the addition of further oxygen and then measuring the SO molecular species at $\mathrm{m} / \mathrm{z} 48$. Detection limits were 0.033 and $1.43 \mu \mathrm{g} \mathrm{L}^{-1}$ for $\mathrm{Au}$ and $\mathrm{S}$ respectively. The internal standard used was Sc.

An interesting paper by Elzey et al. ${ }^{191}$ discussed a method that was capable of quantitative characterization of suspended gold nanoparticles. A custom built electrospray differential mobility analyzer was coupled directly with a condensation particle counter and an ICP-MS instrument. The electrospray differential mobility analyzer achieved real-time upstream size discrimination whilst the particle counter and ICP-MS detectors yielded information on the number density and elemental composition. The key development was a gas exchange device, the design of which was optimized, to improve the conversion of air flow from the electrospray to argon flow that is necessary to sustain the plasma. This proof of concept paper provided a working prototype instrument that was used to achieve several goals during one analytical run.

Another paper has adopted a more theoretical approach to the analysis of nanoparticles. Although it is not strictly an application and should therefore be reviewed elsewhere in other ASU reviews, it is important because it highlights some of the errors that are possible. Brief mention of it will, therefore, be made in this update. Murtazin et al., ${ }^{192}$ discussed sample introduction and plasma-related matrix effects in ICP spectrometry. Use of single monodisperse microdroplets of analyte solution or nanoparticles with a narrow size distribution enabled the provision of quantitative information on the total atomization process (when viewed end-on) as well as spatial information on the process (side-on viewing). Significant spatial shifts of the position of analyte atomization were observed and these were dependent upon the injector gas flow, droplet size, analyte mass, the mass of accompanying elements, etc. The nature of the analyte itself could also be important, with ceramic-like nanoparticles such as titanium dioxide possibly causing more problems than a relatively volatile nanoparticle, e.g., copper or zinc oxide. This was not really addressed in the paper, although mention was made that less volatile analytes are more sensitive to these changes. Although the experiments were performed 
using optical emission, they are also directly relevant to ICP-MS because of the potential requirement of moving the position of the sampling cone, etc.

2.3.5.2. Single particle analysis. It is well known that single particle analysis requires an extremely rapid acquisition time (i.e., the dwell time must be very low) and that very dilute samples are required for analysis. This is to help reduce the possibility of more than one particle being analysed simultaneously. It is, therefore, assumed that a detected pulse corresponds to a single particle event. The number of pulses is directly correlated with the number of particles present and the intensity of the pulse (i.e. the signal height) is proportional to the size of the nanoparticle. Problems can be experienced though since the particles are generally assumed to be spherical. If they are nanowires or irregular in shape, estimation of the particle number concentration from the mass concentration, particle density and shape is difficult. However, if a few assumptions are made so that an estimation of particle number concentration is obtained, it is possible to make an approximation of the particle size from the size of the analyte signal. There is still some debate as to how successful this approach is, but numerous research groups have attempted to provide some insight.

A paper by Mitrano et al. ${ }^{182}$ discussed the use of two of the most powerful methods available for the analysis of engineered nanoparticles; i.e., single particle characterization and $A 4 F$ for the analysis of silver nanoparticles. A range of dwell times between 0.1 and $20 \mathrm{~ms}$ were tested with $10 \mathrm{~ms}$ being optimal, although the data were shown in an electronic supplement rather than in the paper itself. With very rapid dwell times, the possibility of more than one particle being detected at the same time is decreased, but the precision of the determination can be affected adversely. The paper discussed the operational parameters of both techniques and led the reader through their optimization. The two techniques were compared for their size and concentration detection limits, resolution and their multi-form elemental analysis capabilities. Detection limits in terms of particle number and on a mass basis were significantly better for the single particle approach, with LOD at the $\mathrm{ng}^{-1}$ level being obtained compared with the A4F which had LOD at the $\mu \mathrm{g} \mathrm{\textrm {L } ^ { - 1 }}$ level. The size resolution was, however, far superior for A4F-ICP-MS, with detection of $2 \mathrm{~nm}$ particles being possible rather than $20 \mathrm{~nm}$ as obtained using single particle ICP-MS. The single particle approach was, however, capable of differentiating between the dissolved, ionic $\mathrm{Ag}$ signal and nanoparticulate $\mathrm{Ag}$ which the A4FICP-MS was not. The dissolved $\mathrm{Ag}$ would manifest itself as an increase in the number of low intensity pulses. The dynamic range for single particle-ICP-MS is limited in that if too many particles are present, more than one will be detected at any instant. This problem is not suffered by the A4F-ICP-MS approach. Finally, the potential of the two techniques to determine more than one analyte was compared. The A4F-ICP-MS was very capable of this but the single particle approach was not.

The single particle approach to analysing nanoparticles presents many challenges and must be optimized carefully. Reed et al. ${ }^{193}$ have produced a paper entitled "overcoming challenges in analysis of polydisperse-metal-containing nanoparticles by single particle inductively coupled plasma-mass spectrometry".
These workers used zinc oxide, cerium oxide and titanium dioxide nanoparticles as well as silver nanowires during their study. At the sub-ppb detection required, the zinc oxide particles dissolved and hence the single particle approach was unsuccessful. However, the other three materials were determined successfully. Other factors that have to be taken into account to obtain a successful analysis include: the percentage of metal in each nanoparticle, the abundance of each isotope used and the possibility of mass interferences. For instance, the Ce content represents over $80 \%$ of cerium oxide. This means that it would potentially give a higher signal than the Ti, which represents only $60 \%$ of titanium dioxide. Similarly, the $\mathrm{Ag}$ content of silver nanowires is close to $100 \%$ and therefore, easily gives signals above background levels. The Ce signal is far higher for the ${ }^{140} \mathrm{Ce}$ than for any other isotope, simply because its abundance is $88.48 \%$. However, for the Ti determination, the more abundant isotopes cannot be used because they suffer from interferences and so an isotope $\left({ }^{49} \mathrm{Ti}\right)$ that has an abundance of only $5.41 \%$ was used. A $10 \mathrm{~ms}$ dwell time was sufficiently low to minimize the possibility of particle coincidence. However, the authors did note that this particle coincidence may be minimized relatively more easily in standard solutions where the particle size distribution is closely controlled than in "real" samples where they are usually much more diverse. The results obtained using the single particle approach were compared with those obtained using sedimentation field flow fractionation (FFF) and were in good agreement. For the titanium dioxide nanoparticles, the single particle approach yielded a modal diameter of $162 \mathrm{~nm}$ whereas the sedimentation FFF modal diameter was closer to $122 \mathrm{~nm}$. The shape of the distribution was, however, similar for both techniques. Comparison of data obtained using a combination of multiple analyses of dilute solutions with those obtained using a single analysis at higher concentration enabled discrimination between coincidence and polydispersity.

A paper by Pace et al. ${ }^{194}$ discussed some of the challenges experienced when comparing the sizing of four silver nanoparticle dispersions (nominal diameter 40, 60, 80 and $100 \mathrm{~nm}$ ) using the single particle approach with data obtained using dynamic light scattering, differential centrifugal sedimentation, nanoparticle tracking analysis and TEM. The results obtained demonstrated that the single particle approach provided data comparable to the other commercial techniques and with similar accuracy. The single particle analysis approach was then applied to the determination of nanoparticles suspended in algal growth media. An approach to evaluating particle coincidence similar to that described above was also employed in this study.

Hydrodynamic chromatography coupled on-line with single particle ICP-MS was described by Pergantis et al. ${ }^{195}$ The system was applied to the analysis of gold nanoparticles and yielded data on the nanoparticle size, number concentration and metal content. To obtain these data, three calibration series were required: to convert the ICP-MS signal spikes into nanoparticles injected, nanoparticle retention time to nanoparticle size, and ions detected per pulse to metal content in each nanoparticle. The methodology was applied to $60 \mathrm{~nm}$ diameter gold 
nanoparticles spiked into drinking water at a concentration at the $\mathrm{ng} \mathrm{L}^{-1}$ level. The LOD was $2.2 \mathrm{ng} \mathrm{L}^{-1} \mathrm{Au}$, which represented approximately 600 nanoparticles per $\mathrm{mL}$.

As discussed previously, there is a potential problem in single particle-ICP-MS analysis in that most models assume that the particles are spherical. Irregular shaped particles make the calculation of the mass introduced difficult. A paper by Olesik and Gray ${ }^{196}$ discussed the determination of the number of particles and the mass in each of the particles. These authors used the tried and tested protocol of using an ultrasonic bath on bulk materials in an attempt to break agglomerations of particles. Even after dilution, the dilute samples were placed in a sonic bath for two hours prior to analysis. From personal experience, this reviewer has found that such an approach is a help, but that use of a sonic probe is much more efficient. Different dwell times were tested and the precision and likelihood of two particles coinciding were calculated. These authors also found that the dynamic range of the particle number concentration was limited because of the potential coincidence of more than one particle. Several approaches were discussed for the calibration of the number of particles detected as a function of the number of particles per $\mathrm{mL}$ and of the calibration of the signal from a single particle as a function of analyte mass.

Franze et al. ${ }^{197}$ evaluated three different sample introduction devices for the single particle-ICP-MS characterization of silver nanoparticles. These three devices were: a micronebulizer with a Peltier-cooled spray chamber (system one), a micronebulizer with a cyclonic spray chamber and three stage Peltier-cooled desolvation system (system two) and a monodisperse droplet generator with a spray chamber made in-house (system three). Both monodisperse and polydisperse silver nanoparticles of diameter 20-100 $\mathrm{nm}$ were introduced. The authors demonstrated that single $30 \mathrm{~nm}$ nanoparticles could be detected using system one, whereas systems two and three could detect $20 \mathrm{~nm}$ particles. This improvement was attributed to the enhanced sample transport efficiency associated with these two devices. Detection of a $20 \mathrm{~nm}$ silver nanoparticle represents detection of 44 attograms of $\mathrm{Ag}\left(0.03 \mu \mathrm{g} \mathrm{L} \mathrm{L}^{-1}\right)$. Detection efficiencies were also tested. Since the precision was relatively poor, no significant difference could be observed between the three devices. The particle size measurements were validated by data obtained using TEM. The paper went on to differentiate between ionic (dissolved) Ag and signals arising from nanoparticulate silver.

Aureli et al. ${ }^{198}$ used an ICP-MS instrument to investigate the agglomeration and dissolution of silica nanoparticles. Polyatomic interferences on the ${ }^{28} \mathrm{Si}$ isotope arising from $\mathrm{CO}$ and $\mathrm{N}_{2}$ were overcome by introduction of methane at a flow rate of $0.8 \mathrm{~mL}$ $\mathrm{min}^{-1}$ into the dynamic reaction cell. Four different silicon dioxide materials were used and had different agglomeration rates. Some particles formed large clusters of up to $800 \mathrm{~nm}$ in size and, for one material, nearly $51 \%$ was between 91 and $800 \mathrm{~nm}$ with a further $43 \%$ between 21 and $90 \mathrm{~nm}$. The ICP-MS results were confirmed by others obtained using membrane filtration with selective cut-offs. Both SEM and TEM were also used to obtain primary particle sizing and size distribution data.

One other paper, by Tuoriniemi et al. ${ }^{199}$ is of interest in this section of the review. These authors used silver nanoparticles of between 20 and $80 \mathrm{~nm}$ diameter to investigate the detection capabilities of single particle-ICP-MS. An algorithm was developed that enabled particulate signal to be distinguished as outliers from the more continuous dissolved ion signal. If the measured intensity was greater than five times the standard deviation of the whole data set, then the signal was attributed to that of a particle. The smallest particle size that could be detected was $20 \mathrm{~nm}$ and this was attributed to the overlap of particle events and the signal from and dissolved ions. At such low concentrations the noise increases for both signals effectively making them a single event. The optimal dwell time for particles in the 40 to $80 \mathrm{~nm}$ range was $5 \mathrm{~ms}$. The lowest measurable number concentration was limited by the relative frequency of erroneously identified particle events. This number could be reduced by acquiring more data points. The potential of single particle-ICP-MS was then demonstrated using a wastewater treatment plant effluent.

\subsection{Nuclear materials}

There have been a large number of nuclear applications in this review period. Consequently, those that are environmentallybased have largely been omitted in this update in favour of those papers that describe more relevant applications.

2.4.1. Reviews, overviews and CRMs. Several papers have reported the preparation of CRMs or the results of round-robin tests. Amongst this number is a paper by Merchel et al. ${ }^{200}$ who reported the results of an international round-robin exercise for the determination of ${ }^{10} \mathrm{Be}$ using AMS. Three samples covering ${ }^{10} \mathrm{Be} /{ }^{9} \mathrm{Be}$ ratios in the range $10^{-12}$ to $10^{-14}$ were sent to 10 laboratories. To avoid discrepancies arising through the use of different calibration materials, all of the results were made traceable to the CRM NIST 4325. This means that the data obtained for the samples provided gave an indication of any errors during the measurement step alone. Multivariate statistical analysis of the data produced indicated that the participants were divided into two distinct groups with the maximum discrepancy between different laboratories being between 6 and $31 \%$, depending on the ${ }^{10} \mathrm{Be} /{ }^{9} \mathrm{Be}$ ratio. The authors concluded that such exercises are very helpful tools for improving accuracy and precision of AMS data. Varga et al. ${ }^{201}$ described preparation methodology for a reference material to be used for the determination of the production date of uranium materials based on the ${ }^{230} \mathrm{Th} /{ }^{234} \mathrm{U}$ radiochronometer. The reference material was prepared from highly enriched uranium by complete separation of thorium decay products (as proved by gamma spectrometry) followed by addition of a ${ }^{232} \mathrm{Th}$ tracer. The validation of the methodology was achieved by subsequent measurement of ${ }^{230} \mathrm{Th} /{ }^{234} \mathrm{U}$ at recorded times following the last chemical separation. The material produced could be used as a quality control measure for age determination of uranium in nuclear forensics and safeguards as well as for method validation. A further CRM was discussed by Mathew et al. ${ }^{202}$ who used TIMS to determine the isotope abundance ratios of natural uranium in the New Brunswick Laboratory certified reference material $112 \mathrm{~A}$.

Several reviews and overviews have also been published. Included in this number is one by Caroli $e t$ al. ${ }^{203}$ who reviewed 
(with 42 references) the performance of ICP-MS and radiometric techniques (liquid scintillation counting and alpha and gamma spectrometry) for assessing radioactivity in drinking water. The LOD of each of the methods was discussed and the major merits and drawbacks discussed. The review used data obtained from within the authors' own laboratories as well as those in the literature to form their conclusions. Thakur and Mulholland ${ }^{\mathbf{2 0 4}}$ provided a review of the analytical methodology available for the determination of ${ }^{237} \mathrm{~Np}$ in environmental and nuclear samples. The review, which contained 227 references, discussed methods for the separation of ${ }^{237} \mathrm{~Np}$ from the matrix components and from other radioisotopes. Among the methodologies discussed were ion exchange resin separations, liquid-liquid extractions and co-precipitation methods. As well as a great deal of useful text, the review also has an easy reference table that gives the salient points of many of the papers it cites. In addition to atomic spectrometric techniques, the review also covers those applications that use radiometric detection. A review of spectrometric techniques (including FAAS, ETAAS, ICP-OES and ICP-MS) and sample preparation protocols for the determination of trace impurities in nuclear fuel grade uranium was provided by de Souza et al. ${ }^{205}$ Containing 65 references, the review is split into several sections including an introduction, sample decomposition and preparation protocols, strategies employed for matrix separation and analyte preconcentration and individual sections on each of the above detection techniques. An easy reference table of some of the applications is also included in the review.

2.4.2. Nuclear safeguards and forensics. There are increasing concerns over the illicit production and trafficking of nuclear materials. One of the most valuable approaches to combating this is the chronometric investigation (age-dating) of materials. A beginner's guide to uranium chronometry in nuclear materials and safeguards was presented by Stanley. ${ }^{206}$ This author stated that a well-designed chronometric scheme may provide information on a sample's composition, enrichment history and the time lapsed since its last purification. This beginner's guide provided the fundamental concepts of uranium chronometry along with several unique concerns and recent applications. Since this is an expanding area of research, several areas of potential future development were also discussed.

Marin et $a .^{207}$ used LA-SF-ICP-MS for the determination of U isotope ratios in a highly enriched uranium sample. The measurements were improved by optimization of RF power, laser beam diameter, defocusing of the laser beam, laser energy, laser energy density, auxiliary gas and sample gas. The measurements were performed using continuous ablation with low energy density and defocussing since these provided optimal signal stability. A ${ }^{235} \mathrm{U} /{ }^{238} \mathrm{U}$ ratio of $16.36 \pm 0.15$ was obtained with a precision of $1.12 \%$ RSD. The results were compared with those obtained during a laboratory round robin and differed from the average ratio by $0.46 \%$. Comparison with the value assigned by the manufacturer of the material indicated a difference of $0.41 \%$. The data were therefore in sufficiently good agreement for the authors to conclude that LA-ICPMS using a SF instrument offers a rapid and accurate alternative for the determination of $\mathrm{U}$ isotope ratios. The procedure has the advantage of not requiring sample preparation, using the minimum of sample and hence preserving sample integrity.

Kraiem et al. ${ }^{208}$ reported the development of single particle uranium oxide reference materials with certified values for $\mathrm{U}$ content and for isotope ratios. The material was prepared at the European Commission's Institute for Transuranium Elements in Karlsruhe, using an aerosol generation technique developed in-house (the method was discussed in the paper). The paper also described the analysis of the mono-dispersed, micron-sized particles of uranium oxide using ID-TIMS. Although some agglomerates were formed during the generation process, it was only the single particles that were used during the analysis. Single particles (or a maximum of seven) were placed on a predegassed and carburized rhenium filament using a micromanipulator. The particles strongly adhered to the filament without the need for a fixing agent and this was important since any additional materials (i.e., a fixative) may have U contamination and could potentially have distorted the isotope ratios. The single particles were analysed alternately with certified solutions. A spike concentration of $2.2 \mathrm{pg} \mu \mathrm{L}^{-1}{ }^{233} \mathrm{U}$ prepared from the reference material IRMM-058 was used and this was introduced using a micropipette in volumes of between 1 and $4 \mu \mathrm{L}$, depending on the number of particles introduced per filament. The study demonstrated a clear correlation between the particle volume and the $\mathrm{U}$ mass and that the mass could be measured accurately with a relative expanded uncertainty of approximately $10 \%$ (where the coverage factor was $k=2$ ). The production of such materials would be directly applicable for analyses using TIMS, SIMS, LA-ICP-MS. It was noted, however, that some batches of sample had voids in the particles. This was concluded because although the particles were the same size as others, there was significantly less U present. The particles with voids could readily be identified using SEM and so could be removed.

Plutonium dioxide particles may also be used as a quality control measure for safeguards purposes. Shinonaga et al. ${ }^{209}$ first used a chemical separation of americium and then produced such particles using an atomizer system on the New Brunswick Laboratory material CRM-136 solution. The highest density of the size distribution of 312 particles on a selected impactor stage was 0.7 to $0.8 \mu \mathrm{m}$. Analysis of the particles using TIMS, ICP-MS and alpha spectrometry enabled the isotopic composition of $\mathrm{Pu}$ and $\mathrm{U}$ and amount of Am to be estimated. The isotopic composition was, within uncertainty, in agreement with expected values which had been derived from the decay correction of the $\mathrm{Pu}$ isotopes in the original solution. The elemental ratio of $\mathrm{Am}$ to $\mathrm{Pu}$ was determined on the 317th and 674th day after the Am separation. Analysis of single particles using micro-Raman-scanning electron microscopy with EDX detection indicated that the particles produced were plutonium dioxide. However, the authors did note that their initial attempts to measure the density of two single particles provided results with a spread of values accompanied by a large uncertainty.

2.4.3. Other nuclear applications. There have been a large number of other applications during this review period. This section will be split into several paragraphs, each dealing with a 
technique. In addition, a table of some of the applications is included (Table 2).

The first of the techniques to be discussed will be XRF. Several versions of $X R F$ have been employed to analyse nuclear materials. Sato et al. ${ }^{210}$ analysed remelted Zircaloy cladding material. The major elements $(\mathrm{Cr}, \mathrm{Fe}, \mathrm{Ni}, \mathrm{Sn}$ and $\mathrm{Zr})$ were determined using EDXRF whereas the Hf and contaminants were determined using WDXRF. The remelted material had elevated $\mathrm{Cu}, \mathrm{Fe}$ and $\mathrm{Cr}$ levels when compared with the original material. This was attributed to the contamination from iron scraps (for the $\mathrm{Fe}$ and $\mathrm{Cr}$ ) whereas the excess $\mathrm{Cu}$ was thought to originate from contact of the molten Zircaloy droplets with the copper crucible. A TXRF technique was applied by Dhara et al. ${ }^{211}$ for the determination of $\mathrm{Cl}$ in nuclear fuel materials such as $\mathrm{U}_{3} \mathrm{O}_{8}, \mathrm{Pu}$ alloys, $\mathrm{PuO}_{2}$ and $(\mathrm{U}, \mathrm{Pu}) \mathrm{C}$. Sample $(500 \mathrm{mg}$ ) was weighed into a quartz boat which was then placed in a pyrohydrolysis unit. The use of this unit was described in the paper and a schematic diagram was given. Essentially, a mixture of argon and oxygen containing water was passed into the apparatus and the system was heated to $900{ }^{\circ} \mathrm{C}$. The sample breaks down and volatile components, e.g. B, $\mathrm{Cl}, \mathrm{F}$ and $\mathrm{S}$, were liberated and transported by the gas flow through a condenser unit and collected in $5 \mathrm{mM} \mathrm{NaOH}$. After dilution to $25 \mathrm{~mL}$ and the addition of a Co internal standard, aliquots $(30 \mu \mathrm{L})$ of the sample was analysed using TXRF. The $\mathrm{Cl} \mathrm{K} \alpha$ line at $2.622 \mathrm{keV}$ was used to excite the analyte and the fluorescence was measured using a $\mathrm{Si}(\mathrm{Li})$ detector. The sample mass, gas flow rates, pyrolysis temperature and other parameters were all optimized. Precision was quite poor (27\% RSD) when the TXRF determination was undertaken in an air atmosphere. This could, however, be improved to 8\% RSD if He was used instead. Data obtained using pyrohydrolysis-TXRF were compared with those obtained using ion chromatography and were in reasonable agreement, with the TXRF-ion chromatography concentration data having a ratio of between 0.68 and 1.17. The same research group used synchrotron-induced EDXRF to determine $\mathrm{Th}$ and $\mathrm{U}$ in mixed uranium-thorium oxide pellets. ${ }^{212}$ The uranium and thorium oxides were mixed in varying ratios such that the $\mathrm{U}$ content ranged from 0 to $85 \%$. A fixed amount of $\mathrm{Y}$ in the form of yttrium oxide was added as an internal standard and boric acid was added to give a final mass of the mixed material of $2.5 \mathrm{~g}$. The analytes were determined at the $\mathrm{U} \mathrm{L} \alpha$, Th $\mathrm{L} \alpha$ and $\mathrm{Y}$ $\mathrm{K} \alpha$ lines respectively. Precision of the determinations was $0.3 \%$ RSD for U and $0.2 \%$ RSD for Th. The results obtained deviated from the expected amount by 1 and $0.9 \%$ for $\mathrm{U}$ and $\mathrm{Th}$, respectively.

Other workers have used ICP-OES to analyse nuclear materials. The trace components of uranium zirconium alloy were determined using ICP-OES by Sengupta et al. ${ }^{33}$ who digested the material using nitric acid and then selectively extracted the matrix components into an organic phase comprising $30 \%$ tributyl phosphate and carbon tetrachloride. The analytes were then determined in the raffinate. The optimal acid concentration was $7 \mathrm{M}$. Separation of the matrix components was necessary because they are both very line rich, i.e. their presence at an appreciable concentration could interfere with the determination of the analytes. A systematic study of the potential interference posed by these metals on the determination of the analytes (Al, B, Cd, Co, Cr, Cu, Dy, Eu, Fe, Gd, Mn, Na, Ni, Sm and $\mathrm{Zn}$ ) was undertaken and $10 \mu \mathrm{g} \mathrm{mL} \mathrm{m}^{-1} \mathrm{U}$ and $1000 \mu \mathrm{g} \mathrm{mL} \mathrm{L}^{-1} \mathrm{Zr}$ could be tolerated. Five successive extractions were required to ensure that sufficient matrix components were removed. The same research group also published a paper that discussed the development of the methodology for the determination of Am and Th using ICP-OES and their inter-element effects. ${ }^{213}$ Two emission lines for Am were identified: 283.236 and $408.930 \mathrm{~nm}$ providing LOD of $5 \mathrm{ng} \mathrm{mL} \mathrm{m}^{-1}$ and $11 \mathrm{ng} \mathrm{mL} \mathrm{m}^{-1}$, respectively. However, the $283.236 \mathrm{~nm}$ line does suffer from a significant interference by high concentrations of thorium. Three thorium lines were also identified $(283.273,283.730$ and $401.913 \mathrm{~nm})$. The first and last had similar LOD (18 and $13 \mathrm{ng} \mathrm{mL}^{-1}$ ), but the $283.730 \mathrm{~nm}$ line was less impressive, with a LOD of $60 \mathrm{ng} \mathrm{mL} \mathrm{mL}^{-1}$. The authors also discovered that even a small amount of Am contributes significantly to the Th signal at $283.242 \mathrm{~nm}$. Overall, it was concluded that in the presence of Am, Th should be determined using the $401.930 \mathrm{~nm}$ line. Krishnakumar et al. ${ }^{214}$ determined impurities in $\mathrm{PuO}_{2}$ by dissolution of the material and then passing the digest through XAD-4 resin impregnated with trioctyl phosphine oxide. The Pu was retained on the resin whereas the analytes passed through. The $\mathrm{Pu}$ was therefore separated from the impurities.

Several research groups have used LIBS as a means of analysis. This is presumably because LIBS provides the capability of "standoff" analysis (thereby decreasing the exposure of the analyst to potentially harmful samples), is readily automated, and requires only small sample amounts with minimal damage, etc. These were certainly some of the advantages listed in a paper by Martin et al. ${ }^{215}$ who explored its use for the analysis of nuclear materials and in situ applications. These authors used a frequency doubled Q-switched Nd:YAG laser $(532 \mathrm{~nm})$ with $50 \mathrm{~mJ}$ per pulse energy to determine LOD for Cs and $\mathrm{Sr}$ (common nuclear fission products) as well as Ce (used as a surrogate for plutonium in laboratory studies) that had been spiked at different concentrations in calcium carbonate and carbon pellets. The method of pellet preparation was given in the paper and the authors also studied the sampling errors, reproducibility and accuracy of the measurements. Light emitted from the sample was transmitted via a fiber optic to an echelle spectrometer that covered the wavelength range of 200 to $950 \mathrm{~nm}$ and an intensified CCD detector. The spectra obtained from the samples were interrogated using the chemometric techniques of PCA and projection to latent structures. These chemometric techniques enabled the measurements to be made quantitative.

A feasibility study of fusion vessel inner wall chemical analysis using LIBS was undertaken by Almaviva et al. ${ }^{216}$ The LIBS system was described in detail and consisted of a Nd:YAG laser operating at $1064 \mathrm{~nm}$ that could deliver $1 \mathrm{~J}$ of energy in a $8 \mathrm{~ns}$ pulse at a repetition rate of $10 \mathrm{~Hz}$. The laser head was positioned $220 \mathrm{~cm}$ from the sample surface and the laser beam was focused using a lens. Operating at $645 \mathrm{~mJ}$ the laser produced a crater with an area of $2.6 \times 10^{-2} \mathrm{~cm}$, meaning that a fluence of approximately $25 \mathrm{~J} \mathrm{~cm}^{-2}$ was obtained. Light produced was transmitted to the spectrometer by a bundle of 12 fibre optics 
Table 2 Nuclear applications

\begin{tabular}{|c|c|c|c|c|}
\hline Element & Matrix & $\begin{array}{l}\text { Technique; atomization; } \\
\text { presentation }\end{array}$ & Comments & Ref. \\
\hline $\mathrm{Nd}$ & Nuclear fuel & OES; ICP; L, MS; ICP; L & $\begin{array}{l}\text { A cross validation of analytical } \\
\text { procedures (ICP-OES and SF-ICP-MS) for } \\
\text { the determination of Nd. A high } \\
\text { efficiency sample introduction device } \\
\text { was used for ICP-OES. Ten most sensitive } \\
\text { wavelengths monitored, all of which } \\
\text { yielded LOD of below } 1 \mu \mathrm{gg}^{-1} \text {. This was } \\
\text { an order of magnitude lower than had } \\
\text { previously been obtained. In addition, } \\
\text { lower sample consumption ( } 0.3 \mathrm{~mL} \\
\text { min }{ }^{-1} \text { ) was achieved. External } \\
\text { calibration and standard additions used. } \\
\text { SF-ICP-MS data used to confirm accuracy. }\end{array}$ & 223 \\
\hline $\mathrm{Pu}$ & $\begin{array}{l}\text { Analytical laboratory } \\
\text { waste }\end{array}$ & OES; ICP; L & $\begin{array}{l}\text { Hollow fibre supported liquid membrane } \\
\text { technique using } 30 \% \text { tributyl phosphate- } \\
\text { n-dodecane used to extract and } \\
\text { preconcentrate analyte. The two-stage } \\
\text { technique was fast with } 96 \% \text { of } \mathrm{Pu}^{\mathrm{IV}} \text { and } \\
\mathrm{U}^{\mathrm{VI}} \text { extracted from a mixture containing } \\
3.22 \mathrm{~g} \mathrm{~L}^{-1} \mathrm{Pu}, 110 \mathrm{~g} \mathrm{~L} \mathrm{~L}^{-1} \mathrm{U} \text { and } 60.2 \mathrm{mg} \\
\mathrm{L}^{-1} \mathrm{Am} \text { within two hours. The } \mathrm{Am} \\
\text { contamination was less than } 0.1 \% \text {. In the } \\
\text { second stage, the Pu was reduced to Pu }{ }^{\mathrm{III}} \\
\text { and the } \mathrm{U}^{\mathrm{VI}} \text { was selectively transported } \\
\text { into the receiver phase. Separation of } \mathrm{Pu} \\
\text { and } \mathrm{U} \text { therefore achieved. }\end{array}$ & 225 \\
\hline $\mathrm{U}$ isotope ratios & Uranium containing & SIMS;一; S & Fission track technique used as sample & 227 \\
\hline
\end{tabular}

particles

preparation method. A polycarbonate

film containing particles was irradiated with thermal neutrons and etched with 6 $\mathrm{M} \mathrm{NaOH}$ solution. Each particle was identified by observing fission tracks and then that portion of the film was cut and placed on a glassy carbon planchet. Swipe samples containing natural (NBL CRM 950a) or enriched uranium (NBL

CRM U100) had isotope ratios accurately determined. Method then applied to real samples from a nuclear facility. Ratios higher than those obtained using SIMS alone were obtained. 
Table 2 (Contd.)

\begin{tabular}{|c|c|c|c|c|}
\hline Element & Matrix & $\begin{array}{l}\text { Technique; atomization; } \\
\text { presentation }\end{array}$ & Comments & Ref. \\
\hline $\mathrm{Xe}$ & $\begin{array}{l}\text { Uranium dioxide } \\
\text { pellets }\end{array}$ & SIMS;-; S & $\begin{array}{l}\text { Xenon was implanted at two fluences } \\
\left(10^{15} \text { and } 10^{16} \text { atoms } \mathrm{cm}^{-2}\right) \text { in two } \\
\text { batches of uranium dioxide pellets that } \\
\text { differed in grain size ( } 7 \text { and } 22 \mu \mathrm{m}) \text {. } \\
\text { Pellets then treated at } 1673 \mathrm{~K} \text { or } 1873 \mathrm{~K} \text {. } \\
\text { Depth profiles of Xe determined using } \\
\text { SIMS. Diffusion coefficient for the low } \\
\text { fluence was much lower than that found } \\
\text { in the literature. }\end{array}$ & 228 \\
\hline Various (9) & $\begin{array}{l}\text { Structural materials } \\
\text { for a nuclear reactor }\end{array}$ & MS; ICP; LA & $\begin{array}{l}\text { Molten LiF-NaF salts at } 680^{\circ} \mathrm{C} \text { may cause } \\
\text { structural materials of heat exchangers to } \\
\text { become attacked causing micro- } \\
\text { structural and compositional changes. } \\
\text { These corrosion-influenced zones were } \\
\text { studied using LA-ICP-MS and the } \\
\text { analytes Cr, Fe, Mn, Mo, Ni, Ti, W as well } \\
\text { as Li and Na determined semi- } \\
\text { quantitatively. Corrosion products } \\
\text { determined in coolant after dissolution } \\
\text { using ICP-OES. }\end{array}$ & 229 \\
\hline $\begin{array}{l}\text { Various }(\mathrm{K}, \mathrm{Li} \\
\text { and } \mathrm{Na})\end{array}$ & $\begin{array}{l}\text { Samples from } \\
\text { pyrochemical } \\
\text { re-processing }\end{array}$ & OES; ICP; L, MS; ICP; L & $\begin{array}{l}\text { Comparison of ICP-OES and ICP-MS } \\
\text { similar to } \\
\text { samples employed to assess the accuracy. } \\
\text { No internal standard required. }\end{array}$ & 230 \\
\hline Various (REEs) & $\begin{array}{l}\text { Nuclear grade } \\
\text { uranium oxide }\end{array}$ & OES; ICP; L, MS; ICP; L & $\begin{array}{l}\text { Solid phase extraction of analytes as their } \\
\text { fluorides onto activated charcoal. } \\
\text { Residual U content in solution was }<10 \\
\mu \mathrm{g} \mathrm{mL}^{-1} \text {. Recovery of REEs ranged from } \\
85 \text { to } 105 \% \text {. Method validated using } \\
\text { uranium oxide reference materials. }\end{array}$ & 231 \\
\hline
\end{tabular}

and some lenses. The spectrometer itself comprised three different gratings with 1200, 2400 and 3600 grooves $\mathrm{mm}^{-1}$ and had a resolution of approximately $0.01 \mathrm{~nm}$ at $500 \mathrm{~nm}$. The detector was an intensified CCD that had a minimum gating time of $10 \mathrm{~ns}$. The effects of time delay and laser fluence on the LIBS sensitivity at reduced pressure were examined. It was possible to detect thin interfaces on different materials demonstrating that it would be possible to monitor the erosion of the surface and deposition of impurities. A paper, by Ko et $a{ }^{217}$ used femtosecond LIBS to make an initial study of natural uranium. The design and performance of the system were discussed.

Resonance ionization mass spectrometry (RIMS) is regarded as being one of the most sensitive and selective techniques for the determination of long-lived radionuclides. The research group at Mainz has produced three papers of interest during this review period. In one, by Trautmann and Wendt, ${ }^{218}$ the potential of RIMS for determining long-lived $\mathrm{Pu}$ and $\mathrm{Np}$ isotopes, including isotope ratio measurements, was determined. In addition, the first ionization potentials of 10 actinide elements were determined using the photothreshold method. Only $10^{12}$ atoms were required for these measurements. This paper also described the investigation of short-lived fission products. For these studies, a gas jet and its combination with a thermochromatographic separation and a continuous solvent extraction technique were used. The paper discussed all of the examples in detail. A second paper $^{\mathbf{2 1 9}}$ discussed the ultra-trace determination of Tc. The state of the art and the specifications of RIMS-based detection of Tc leading to detection at $3 \times 10^{4}$ atoms level were reported. The third paper in the series discussed the detection of Pu at ultra-trace quantities. According to the authors, the method enabled the unambiguous identification and individual quantification of both ${ }^{238} \mathrm{Pu}$ and ${ }^{241} \mathrm{Pu}$. These isotopes are, of course, of relevance for the dating of radiogenic samples. Since the ionization is selective to individual elements, the presence of large quantities of ${ }^{241} \mathrm{Am}$ and ${ }^{238} \mathrm{U}$ did not cause isobaric interferences. Again, LOD were of the order of $10^{4}$ to $10^{5}$ atoms.

There has been an upsurge in the number of accelerator mass spectrometry (AMS) papers published during this review period. In one paper, Lu et al. ${ }^{220}$ reported their attempts to separate the ${ }^{93} \mathrm{Zr}-{ }^{93} \mathrm{Nb}$ isobar so that ${ }^{93} \mathrm{Zr}$ may be determined accurately using AMS so that it may be used as a tracer in hydrological and radioactive waste studies. Results of the first experiments, involving the use of a combination of a gas-filled magnet with a position-sensitive parallel grid avalanche counter and a gas ionization chamber in the magnet's focal plane, were reported. Although the experiment was not entirely successful, sufficient progress was made to give reassurance that the separation may be possible. Further modifications include the use of a more 
powerful magnet, the reduction of the $\mathrm{Nb}$ and the further optimization of the beam energy and the gas pressure in the gas-filled magnet.

The final two papers to be discussed in this section describe speciation analysis. Stobener et al. ${ }^{221}$ developed a capillary electrophoresis (CE)-ICP-MS technique for the redox speciation analysis of Np. The CE and ICP-MS coupling was via a commercial low flow nebulizer and a Scott-type spray chamber. The CE separated $\mathrm{Np}^{\mathrm{IV}}$ and $\mathrm{Np}^{\mathrm{v}}$, which are the only oxidation states that are stable in the environment. Limits of detection were $1 \times 10^{-9}$ and $5 \times 10^{-10} \mathrm{~mol} \mathrm{~L}^{-1}$ for $\mathrm{Np}(\mathrm{Iv})$ and $\mathrm{Np}(\mathrm{v})$ respectively, which were sufficiently low to be suitable for measurement of the analytes in samples from a nuclear waste repository. A linear calibration range spanning three orders of magnitude $\left(10^{-9}\right.$ to $\left.10^{-6} \mathrm{~mol} \mathrm{~L}^{-1}\right)$ was obtained for both species. The method was applied to the speciation analysis of Np left in solution after interaction of $5 \times 10^{-7} \mathrm{~mol} \mathrm{~L}^{-1} \mathrm{~Np}(\mathrm{v})$ with Opalinus clay. Under oxidizing conditions, a Np sorption of $31 \%$ occurred and all of the Np remaining in solution was in the (V) state. A second experiment in which the $\mathrm{Np}$ was mixed with $\mathrm{Fe}^{2+}$ and then Opalinus clay led to complete sorption. After desorption using perchloric acid, the Np was present in both the (IV) and (V) states. The other paper of interest in this section was prepared by Eichler et al. ${ }^{222}$ who used HPLC-ICP-MS to monitor the mobility of Se in engineering barriers of stored nuclear waste. Since ${ }^{79} \mathrm{Se}$ is a decay product of ${ }^{238} \mathrm{U}$, it is necessary to predict its behavior in the bentonite barriers. The interconversions of non-radioactive Se species were studied in aqueous suspensions of bentonite at various $\mathrm{pH}$ using HPLC with both reversed phase and anion exchange columns. The chromatographic parameters were optimized to improve the separation of the species. In alkaline medium and in the presence of bentonite, some selenite was oxidized to selenate within two weeks. The retention of selenate in the barrier is lower than that of selenite and therefore it was concluded that Se could easily spread.

\section{Materials}

\subsection{Ceramics and refractories}

An overview (with 111 references) of the use of XRF in the analysis of cultural heritage artefacts was given by Musilek et al. ${ }^{54}$ The overview presented a brief description of the instrumentation and went on to discuss applications. Materials covered in the review included ceramics, glasses, paintings and frescos, manuscripts, drawings and prints, metals and other materials such as deposits on buildings etc. Although the paper is clearly not a comprehensive review, it does provide the reader with an indication of the areas that may be addressed using the different XRF-based techniques and gives many relevant references. A brief description of the work undertaken at the authors' institute was also presented.

Ceramics are usually resistant to heat and are often relatively inert meaning that very harsh conditions are usually required for their dissolution. Consequently, many of the methods used for their analysis have taken advantage of the techniques that are capable of analysing solid samples directly. This means that techniques that utilise lasers and X-ray-based methods are the most common. These will be discussed in this section of the review. It should be noted that a significant number of papers describing the preparation of bio-ceramic materials have been published. However, the focus of these papers was almost entirely on the material itself rather than the analysis. They have, therefore, not been reviewed.

A new multi-element analysis technique based on laserexcited atomic fluorescence was discussed by Cai et al. ${ }^{232}$ The authors managed to induce numerous analytes to fluoresce even though only one excitation wavelength $(193 \mathrm{~nm})$ was used. This was only possible if the analytes were embedded in a dense plume, such as that produced during pulsed laser ablation. Full details were given in the paper as was an explanation of the mechanism. Numerous applications were presented including the analysis of metals, plastics, ceramics and their composites. Impressive LODs (ng g ${ }^{-1}$ range) were obtained meaning that absolute amounts at the atto-mole level could be determined. The procedure was, therefore, regarded as being non-sample destructive.

Hall effect thrusters are promising electric propulsion devices that enable geostationary satellites to maintain their station. The main limiting factor of their lifespan is the erosion of the annular channel's ceramic walls caused by energetic xenon impacting on it. An interesting application of LIBS was discussed by Balika et al. ${ }^{233}$ who described the analysis of the ceramic wall to monitor the erosion process whilst the thruster was in operation. The full set-up was described and a schematic diagram was given in the paper. Briefly, the thruster was placed in a vacuum chamber and Xe gas introduced at a rate of $5 \mathrm{mg} \mathrm{s}^{-1}$. A Nd:YAG laser operating at $532 \mathrm{~nm}$, with pulses of $10 \mathrm{~ns}$ duration and energy in the 10 to $80 \mathrm{~mJ}$ range provided a beam spot $300 \mu \mathrm{m}$ in diameter. This was focussed via a lens and a slit machined in the outer wall onto the inner ceramic wall surface. A laser pulse rate of $10 \mathrm{~Hz}$ was used and the number of pulses used was between 50 and 100. The light emitted was transmitted via a fiber optic to a spectrometer and an intensified CCD camera detector. The amount of material ablated had to be determined so that a direct correlation between mass and the optical signal could be obtained. This was achieved off-line using both a mechanical contact profilometer and an optical profilometer. Once the correlation (i.e., a calibration) had been made, it was possible to use the LIBS system remotely to monitor the degradation of the ceramic wall.

Some ceramics are used in the electronics industry. Several papers have, therefore, been published that used techniques capable of surface analysis or depth-profiling to determine the diffusion properties of analytes. Several of these papers will have been discussed elsewhere in the review, but it is worth noting some here for completeness. Idris et al. ${ }^{234}$ used SIMS to monitor the effects of both temperature (between 1073 and $1673 \mathrm{~K}$ ) and oxygen activity on the segregation on the surface and near surface layers of yttria stabilized zirconia. The effect of oxygen was significant over the temperature range 1073-1473 K with the maximum enrichment of Y being observed at $1273 \mathrm{~K}$. Several techniques including SIMS, X-ray photoelectron core level spectroscopy and extended X-ray absorption fine structure 
(EXAFS) were used by Lysaght et al. ${ }^{235}$ to monitor the $\mathrm{Al}$ and $\mathrm{N}$ diffusion in hafnium dioxide and its interface with the silicon substrate in (001) $\mathrm{Si}-\mathrm{SiO}_{x}-2 \mathrm{~nm} \mathrm{HfO}_{2}-1 \mathrm{~nm} \mathrm{AlO}_{x}$ film structures. Diffusion of the $\mathrm{Al}$ was observed from the $\mathrm{AlO}_{x}$ cap layer deposited on annealed and unannealed hafnium dioxide following annealing in nitrogen and at an ambient temperature in ammonia. The authors decoupled the effects of nitrogen from the thermal reactions alone by annealing in both nitrogen and ammonia. Causal variations in the hafnium dioxide microstructure combined with the dependence of $\mathrm{Al}$ and $\mathrm{N}$ diffusion on initial hafnium dioxide conditions were presented with respect to anneal temperature.

Although the majority of applications utilized solid sampling methods, some have managed to undertake a dissolution process prior to analysis. One such example was described by Tagle et al. ${ }^{236}$ who determined metal impurities in advanced lead zirconate titanate ceramics using a microwave-assisted acid dissolution prior to ICP-OES analysis of the digests. Two microwave digestion methods were compared. Both used $0.25 \mathrm{~g}$ of pulverized material, but one used a mixture of hydrochloric and hydrofluoric acids and the other a mixture of nitric and hydrofluoric acids plus hydrogen peroxide. Both then underwent the same microwave assisted dissolution program, followed by addition of perchloric acid, evaporation and then dilution to $50 \mathrm{~mL}$ using $5 \%$ nitric acid. The protocol using hydrochloric acid provided a very low yield of $\mathrm{Pb}$ (approximately $40 \%$ ) and this was attributed to the precipitation of $\mathrm{PbCl}_{2}$. The recoveries of the other major elements were in good agreement with those obtained using the nitric acid dissolution procedure. The nitric acid digestion procedure was, therefore, adopted for subsequent experiments, even though the levels of $\mathrm{Sr}$, Ti and $\mathrm{Zr}$ were between 4 and $9 \%$ lower than the expected values. A suite of trace impurities (13 analytes) was then determined in the digests. The analysis stage was also optimized, with nebulizer flow rate and plasma power being of highest importance in obtaining robust plasma conditions and decreasing matrix effects. Precision for the analytes ranged from 3 to $13 \%$ whilst spiking experiments led to recoveries of between 90 and $110 \%$. The LOD were very variable with the best being $0.004 \mathrm{mg} \mathrm{kg}^{-1}$ (for $\mathrm{Mn}$ ) and the worst $10 \mathrm{mg} \mathrm{kg}^{-1}$ (for $\mathrm{Fe}$ ).

3.1.1. Analysis of archaeological, cultural heritage and art objects. As well as the review discussed previously in Section 3.4.1, ${ }^{54}$ there has been a large number of applications in this area of research. As discussed in previous updates, many of these applications are published in archaeological journals and few give many details of the analytical chemistry. Despite this, these references are interesting because they demonstrate how analytical chemistry can assist in the elucidation of manufacturing procedures, provenance, etc. This is especially true when a chemometric technique is used to analyse the atomic spectrometric data. Since the materials may be precious or delicate, minimal damage must be inflicted on them. Therefore the techniques used for these materials tend to be those that are capable of analysing solid materials directly leaving no visible damage. Some though, do still use a more traditional destructive approach, e.g., acid digestion. Those papers that are of interest but do not discuss the analytical chemistry in detail, are best presented in tabular form (Table 3). Other papers are discussed below.

The potential consequences of ion beam analysis on cultural heritage objects have been appraised by Zucchiatti and AgulloLopez. ${ }^{237}$ Ion beam analysis covers many techniques, e.g. PIXE, RBS, PIGE, elastic recoil detection analysis (ERDA), nuclear reaction analysis (NRA) and others. Although the aim is to cause no damage to the samples, the interaction of the ion beam with the sample does cause some changes. This paper attempted to establish the limits so that damage is minimal or that the analysis is viable in terms of the cost benefit ratio. Interaction of the ion beam with the sample may cause discoloration, heating, sputtering of the surface, displacement of atoms in the sample, changes in the composition arising from implantation of new species produced by nuclear reactions and changes in microscopic structures (because of the formation of new phases). Each of these potential problems was discussed in the paper.

Some studies are worth discussing in more detail. Bardelli et $a .^{238}$ used several synchrotron-based X-ray techniques to elucidate the Fe speciation in samples of ancient Attic pottery from the Gioiosa Guardia archaeological site in the Strait of Messina. The Fe speciation analysis was undertaken using PCA and least-squares fitting procedures applied to the data obtained from the near edge part of the absorption spectra (XANES). Details of the local structure around the Fe sites was achieved using the extended part of the spectra; the technique known as extended X-ray absorption fine structure (EXAFS). The red pigments were comprised of an admixture of hematite or maghemite $\left(\mathrm{Fe}_{2} \mathrm{O}_{3}\right)$ and magnetite $\left(\mathrm{Fe}_{3} \mathrm{O}_{4}\right)$. Different ratios of the material accounted for the dark and lighter coloured areas on the samples. The data obtained in this paper were complementary to those obtained previously using INAA, FTIR and time of flight neutron diffraction. A similar study by Figueiredo et al. ${ }^{239}$ used XANES to study the Co speciation in the blue and white glazes of 16 th and 17 th century Chinese porcelains. Preedge features and edge details were discussed and a comparison made with XANES data obtained from the model compounds $\mathrm{Co}_{3} \mathrm{O}_{4}, \mathrm{CoAl}_{2} \mathrm{O}_{4}$ and $\mathrm{Co}_{2} \mathrm{SiO}_{4}$, which have well known crystal structures. In addition, a blue pigment (cerulean) was also analysed. A WDXRF instrument was also used to determine other trace elements; with the $\mathrm{K} \alpha$ line being used for $\mathrm{Co}, \mathrm{Cu}, \mathrm{Fe}$, $\mathrm{K}, \mathrm{Mn}, \mathrm{Ni}$ and $\mathrm{Zn}$ and the $\mathrm{L} \beta$ line being used for $\mathrm{Sn}$. The Co K-edge XANES data presented confirmed the presence of tetrahedral $\mathrm{Co}^{2+}$ ions being responsible for the blue colouring of the glazes.

Another paper to use synchrotron-based XRF was prepared by Guilherme et $a l^{\mathbf{2 4 0}}$ who used $\mu$-XRF for the determination of trace elements in Portuguese glazed ceramics from the 16th to 18 th centuries. Samples from two sites were analysed. The ones from Coimbra comprised faiences and tiles, whereas those from Lisbon were only tiles. Such pieces contain three parts to their structure: the bulk ceramic, the glaze and the surface decoration. The decoration and glaze are not easy to investigate because of the high degree of heterogeneity resulting from the mixing procedures and the firing temperatures. The extent to which the decoration diffuses into the glaze is dependent on their compositions. The decoration was analysed using the high 
resolution instrumentation and the blue samples were high in $\mathrm{Co}$, the purple ones contained significant amounts of $\mathrm{Mn}$, the yellow ones contained $\mathrm{Sb}$ and the green ones $\mathrm{Cu}$. The glaze contained $\mathrm{Pb}$ whereas the body of the ceramic often contained significant amounts of Fe. A difference was observed between faiences and tiles, with the glaze in the former being typically 150 to $200 \mu \mathrm{m}$ thick whereas the tiles' glaze was typically 350 to $400 \mu \mathrm{m}$ thick. The samples from Lisbon had a higher intake of the pigment in the glaze than those from Coimbra.

\subsection{Thin films and depth profiling}

The development of techniques for the characterisation of thin films continues to be a subject of research in the current review period. Advances are summarised in this section only from those publications that focus specifically on analytical development, or provide insights in relation to comparative performance in the increasingly prevalent multi-technique approach to the characterisation of these materials. In some cases, thin films are deposited on glass substrates. Where the work relates to the characterisation of the film or its relationship with the substrate it is described in this section. Developments in the characterisation of glasses are presented in Section 3.3 of this review.

A six page review of the use of LIBS for the surface analysis of materials has been published by Zhang et al. ${ }^{261}$ Subjects covered include theory, instrumentation, and features of the technique such as elemental distribution and depth profiling. The factors that affect analytical performance such as the selection of the ambient gas and operating pressure, the pulse energy and distribution of the laser beam are also explored. The authors identify the key advantages of LIBS in terms of the ability to scan a large area of sample, and the capacity to analyse conducting and non-conducting samples. Applications in electronic materials, semiconductors and metallurgy are cited.

There have been many reports during the period under review of the use of multiple techniques in the characterisation of thin film materials. For example, the outcome of an interlaboratory comparison of surface analysis techniques for the characterisation of $\mathrm{Fe}-\mathrm{Ni}$ alloy thin films has been published by Kim et al. ${ }^{262}$ This work was carried out under the auspices of Surface Analysis Working Group of the Consultative Committee for Amount of Substance by four National Metrology Institutes and one Designated Institute. Four laboratories used XPS and one used Auger Electron Spectroscopy for the analysis. Unsurprisingly, it was shown that if quantification was carried out using relative sensitivity factors for $\mathrm{Fe}$ and $\mathrm{Ni}$ derived from an alloy reference sample, the results obtained were more accurate than if pure $\mathrm{Fe}$ and $\mathrm{Ni}$ films were used for the same purpose. However, it was noted that the uncertainty budget for the measured chemical composition of the Fe-Ni alloy film on this basis was dominated by the uncertainty of the composition of a reference specimen previously analysed by ICP-MS. The individual expanded uncertainties for the participating laboratories were given in the range 2.88 to 3.40 atomic $\%$.

The development of analytical techniques for investigating the growth of novel material structures remains an area of significant activity. For example, the nucleation of alpha $\mathrm{Al}_{2} \mathrm{O}_{3}$ via $\mathrm{Ti}_{x} \mathrm{O}_{y}$ templates on $\mathrm{Ti}(\mathrm{C}, \mathrm{N})$ coated cemented carbide has been studied using a range of techniques including SEM, XRD, Auger electron spectroscopy and TOF-SIMS. ${ }^{263}$ It was found that the titanium oxide template changed in structure from $\mathrm{Ti}_{4} \mathrm{O}_{7}$ phase to a $\mathrm{Ti}_{3} \mathrm{O}_{5}$ phase during deposition of $\mathrm{Al}_{2} \mathrm{O}_{3}$. The application of Auger electron spectroscopy and particularly SIMS depth profiling demonstrated that this transformation had the effect of creating pores at the $\operatorname{Ti}(\mathrm{C}, \mathrm{N})$ interface, thereby causing a lowering of interfacial strength. The growth of In-Ga-N type films on quartz glass substrates has been investigated using SIMS, XPS and XRD. ${ }^{264}$ The extent of oxygen contamination and the chemical states of the films were studied using XPS while the distribution profile of $\mathrm{O}$ in the films was investigated using SIMS. While these techniques indicated the presence of oxide phases in the entire film, this could not be confirmed using XRD analysis.

The accurate determination of fluorine is often challenging and an evaluation of five spectroscopic techniques for the detection of the element in doped tin oxide films has been published. ${ }^{265}$ Thin films of this type were prepared on cleaned glass substrates by spray pyrolysis using $\mathrm{SnCl}_{4}$ and $\mathrm{NH}_{4} \mathrm{~F}$. The $\mathrm{SnO}_{2} \mathrm{~F}$ doped films were characterised using EPMA, SEM, WDXRF, XPS and RBS. It was reported that acceptable agreement was obtained for the quantitative determination of $\mathrm{F}$ in these films by WDXRF and RBS. It was noted that approximately $90 \%$ of the $\mathrm{F}$ content was lost during the spray pyrolysis deposition process and only $10 \%$ was subsequently embedded within the films.

3.2.1. Laser-based techniques. A calibration-free method for the compositional analysis of an indium-tin-oxide material has been reported using laser induced breakdown spectroscopy. ${ }^{266}$ Indium emission was observed at three different wavelengths (325.609, 410.177 and $451.131 \mathrm{~nm})$ and four Sn lines (283.999, 286.333, 317.502 and $326.234 \mathrm{~nm}$ ) were used for measurements. As might be anticipated, it was reported that the intensity of emission signals for In and Sn were related proportionately to the applied laser energy but that the degree of enhancement observed was greater for In than for Sn. This was attributed to differences in energy levels of the transitions monitored. It was found that the optimal conditions for the analysis involved the use of a $20 \mathrm{~mJ}$ laser pulse (equivalent to a plasma temperature of $5400 \mathrm{~K}$ ) followed by a subsequent 2 to $4 \mu$ s delay prior to the acquisition of spectral data. Under these conditions the content of In and $\mathrm{Sn}$ in the oxide material was reported to be 30 and $32 \%$ respectively. Also in this vein, the elemental analysis of a thin $(1.23 \mu \mathrm{m})$ copper indium gallium diselenide (CIGS) absorption layer has been carried out using LIBS. ${ }^{267}$ The absorption layer was deposited by co-evaporation on the surface of a Mo-coated soda-lime glass substrate. Under optimum analytical conditions, good calibration linearity was achieved for the Ga-In ratio when compared with equivalent data measured using XRF and ICP-OES. (see also Section 3.2.3.1 of this review). The LIBS technique has also been used in the characterisation of thin $\mathrm{Cu}$ films $(1 \mu \mathrm{m})$ on soda lime glass substrates. ${ }^{268} \mathrm{~A}$ nanosecond Q-switched Nd:YAG laser was used to ablate material with a notional spatial resolution of $50 \mu \mathrm{m}$. It was found that when a 
Table 3 Analysis of archaeological, cultural heritage and art objects

\begin{tabular}{lll}
\hline Element & Matrix & $\begin{array}{l}\text { Technique; } \\
\text { presentatio }\end{array}$ \\
\hline Various (16) & $\begin{array}{l}\text { Pottery from Sisak, } \\
\text { Croatia }\end{array}$ & OES; ICP; L
\end{tabular}

Various $(>30)$

Various

Various (12)

Various (18)

Various (10)

Various (13)

(nine major and

four minor

analytes) 17th century Persian $\quad$ XRF;-; S

Haft Rang tiles

Islamic potteries

with a yellow

opaque glaze from

Sinai, Egypt

Varnished pottery

from Syracuse and

Adrano (Sicily)

White slip sherds

from late bronze age

sites in Cyprus and Syria

Ceramic artefacts from Neolithic sites in Serbia

Song dynasty Guan wares and later imitations

Fine ware from three Archaic and Hellenistic kilns in Sicily
XRF;-; S, XRD, SEM-EDX

XRF;-; S, SEM-EDX

MS; ICP; L, XRF;-; S, SEM-EDX

$\mathrm{XRF} ;-; \mathrm{S}$

XRF;-; S

XRF;-; S, XRD, microscopy
Comments

Ref.

High pressure microwave digestion of early Roman materials using only nitric acid. Data validated by analysis of CRMs. Chemometric techniques of principal components analysis (PCA) and cluster analysis enabled differentiation between local and imported goods.

Wavelength dispersive instrument used to determine analytes with PCA analysis of data enabling provenance to be elucidated. All 43 samples analysed were made from local clay. Portable XRF and XRD instruments used. Glaze composed of $\mathrm{Sn}, \mathrm{Sb}$ and $\mathrm{Pb}$. Paper is in Japanese.

Varnished surfaces analysed using nondestructive portable XRF and SEM-EDX. The bulk of the pottery was analysed using microdestructive XRF. Data were verified by analysis of numerous certified rock samples. Bi-plots and ternary diagrams used to identify several differences between varnishes. Syracuse samples identified as being high quality and produced at an advanced level. Adrano samples were poorer quality.

Fragments were acid digested and the $\mathrm{Pb}$ isotopes preconcentrated using an ion exchange resin prior to determination using two multi-collector ICP-MS instruments. Thallium was used as an internal standard. Wavelength dispersive XRF determined other analytes in $300 \mathrm{mg}$ of a dried and homogenised powder of the samples. Analytical data for analytes plus the ratios of ${ }^{208} \mathrm{~Pb} /{ }^{204} \mathrm{~Pb}$, ${ }^{207} \mathrm{~Pb} /{ }^{204} \mathrm{~Pb}$ and ${ }^{206} \mathrm{~Pb} /{ }^{204} \mathrm{~Pb}$ inserted into PCA to facilitate provenance studies.

Portable instrumentation used to ensure non-destructive analysis. The objective was to develop a decision making process to classify the samples. The analytical data were inserted into PCA and scattering matrices-based dimension reduction. The latter provided a better decision making process. Seven out of eight unknown samples were correctly classified. Samples (58) were analysed including 40 authentic Guan ware samples and 18 samples ascribed as being imitation. Analysis using EDXRF and PCA treatment of the data demonstrated that the glaze was significantly different between the Song Guan samples and the imitations. Results indicated that seven samples may have been mis-identified by the traditional method. A brief provenance study was also undertaken. Characterization of 35 samples originating from three kilns and five coming from a Hellensitic house. Ternary plots and PCA used for classification. All samples, except those that had been imported, had the same petrographic features. However, chemical analysis identified two clear groups. 
Table 3 (Contd.)

\begin{tabular}{|c|c|c|c|c|}
\hline Element & Matrix & $\begin{array}{l}\text { Technique; atomization; } \\
\text { presentation }\end{array}$ & Comments & Ref. \\
\hline Various & $\begin{array}{l}\text { Roman double- } \\
\text { layered crucibles } \\
\text { from Autun, France }\end{array}$ & $\begin{array}{l}\text { XRF;一; S, XRD, SEM-EDX, } \\
\text { EPMA }\end{array}$ & $\begin{array}{l}\text { WDXRF and other methods used to } \\
\text { analyse } 18 \text { samples so that information on } \\
\text { the raw materials in the individual layers } \\
\text { could be obtained. The mineral } \\
\text { composition enabled an estimation of the } \\
\text { firing temperature }\left(1200-1400^{\circ} \mathrm{C}\right) \text { to be } \\
\text { obtained. }\end{array}$ & 249 \\
\hline Various (47) & $\begin{array}{l}\text { Ceramic sherds } \\
\text { from Papua New } \\
\text { Guinea }\end{array}$ & MS; ICP, LA & $\begin{array}{l}\text { Analysis of } 287 \text { ceramic sherds used as a } \\
\text { proxy for human interaction on the North } \\
\text { coast of Papua New Guinea. Samples were } \\
\text { analysed using } 10 \times 100 \mu \mathrm{m} \text { spots from } \\
\text { clean edges ensuring large mineral grains } \\
\text { and pore spaces were not included. } \\
\text { Certified glasses NIST } 610 \text { and NIST } 612 \\
\text { and standard clay NIST } 679 \text { were used } \\
\text { every five samples. Jack-knifed } \\
\text { Mahalanobis distance and canonical } \\
\text { discriminant function analysis used to } \\
\text { classify specimens. Despite samples being } \\
\text { exported from Tumleo Island for a } \\
\text { thousand years (indicating human } \\
\text { interaction), the language diversity has } \\
\text { been maintained. }\end{array}$ & 250 \\
\hline Various (33) & $\begin{array}{l}\text { Formative period } \\
\text { ceramics from the } \\
\text { Teotihuacan valley }\end{array}$ & $\begin{array}{l}\text { MS; ICP; LA, TIMS;一; L, } \\
\text { INAA }\end{array}$ & $\begin{array}{l}\text { TIMS used to determine }{ }^{87} \mathrm{Sr} /{ }^{86} \mathrm{Sr} \text { after an } \\
\text { acid dissolution (details given). INAA used } \\
\text { to determine } 22 \text { analytes. LA-ICP-MS used } \\
\text { to target clay rather than mineral } \\
\text { inclusions. Data inserted into PCA and } \\
\text { Mahalonobis distances to obtain } \\
\text { classification. Most pottery was } \\
\text { prepared from local raw materials } \\
\text { and this differed from later } \\
\text { post-classical materials. One } \\
\text { settlement has ceramics made from } \\
\text { distinct raw materials. }\end{array}$ & 251 \\
\hline
\end{tabular}

major, 12 minor analytes)

\section{pottery}

crushed, dried at $700{ }^{\circ} \mathrm{C}$ for 4 hours and then fused with anhydrous lithium tetraborate to form a glass bead (methodology discussed). The bead was then analysed using WDXRF with calibration using the fundamental parameters method and three certified rock and three certified stream sediment samples. The second sample preparation protocol involved powdered sample $(100 \mathrm{mg})$ being transferred to a sample holder and gently pressed (by hand) into a pellet. Calibration was by mixing assorted inorganic components together and grinding to a particle size of less than $10 \mu \mathrm{m}$. Analysis of three reference materials demonstrated validity of the second protocol. Cluster analysis was used for data interpretation. The second sample preparation procedure had the advantage of consuming less sample and also being rapid. 
Table 3 (Contd.)

\begin{tabular}{|c|c|c|c|c|}
\hline Element & Matrix & $\begin{array}{l}\text { Technique; atomization; } \\
\text { presentation }\end{array}$ & Comments & Ref. \\
\hline Various (35) & $\begin{array}{l}\text { Ceramic building } \\
\text { material from } \\
\text { Carpow and York } \\
\text { Roman fortresses }\end{array}$ & MS; ICP; L & $\begin{array}{l}\text { Samples prepared using an acid dissolution } \\
\text { (methodology given). Reference materials } \\
\text { were run to verify data. Raw clay and fired } \\
\text { clay were also analysed and the results } \\
\text { obtained were compared with those from the } \\
\text { ceramic building materials. It was confirmed } \\
\text { that firing does not disturb the elemental } \\
\text { fingerprint. The building materials from } \\
\text { Carpow had the same elemental fingerprint } \\
\text { as those from York, demonstrating long } \\
\text { distance importation. }\end{array}$ & 253 \\
\hline Various & $\begin{array}{l}\text { Xicun Qingbai wares } \\
\text { from Northern Song } \\
\text { dynasty }\end{array}$ & XRF;一; S, MS; ICP; LA & $\begin{array}{l}\text { Major analytes ( } 7 \text { ) determined using EDXRF. } \\
\text { Minor analytes ( } 27+\text { REEs) determined using } \\
\text { LA-ICP-MS. NIST glass samples } 610 \text { and } 612 \\
\text { used for external calibration. Data } \\
\text { interpretation using PCA and scatter } \\
\text { diagrams enabled provenance to be } \\
\text { elucidated. Content of } \mathrm{Ba}, \mathrm{Pb}, \mathrm{Rb}, \mathrm{Ta}, \mathrm{Th}, \mathrm{V} \\
\text { and the Nb-Ta ratio enabled discrimination } \\
\text { between products from the Xicun and } \\
\text { Chaozhu kilns. }\end{array}$ & 254 \\
\hline Various (20) & $\begin{array}{l}\text { Semi-fused } \\
\text { Hellenistic } \\
\text { potsherds from } \\
\text { Harabebezikan, } \\
\text { Turkey }\end{array}$ & XRF;-; S, SEM-EDX, XRD & $\begin{array}{l}\text { Sample finely powdered and fused with } \\
\text { lithium tetraborate (a } 1: 10 \text { ratio) prior to } \\
\text { analysis using WDXRF. Both SEM-EDX and } \\
\text { XRD used to investigate the chemical } \\
\text { compounds present in the samples. The } \\
\text { absence of clay minerals such as illite and } \\
\text { muscovite and the presence of pyroxenes } \\
\text { such as diopside and augite indicate a firing } \\
\text { temperature of at least } 1000^{\circ} \mathrm{C} \text {. This implied } \\
\text { that the samples had been fired in a kiln } \\
\text { rather than in a pit or a bonfire. }\end{array}$ & 255 \\
\hline Various (36) & $\begin{array}{l}400 \text { samples of early } \\
\text { and middle bronze } \\
\text { age Cypriot pottery }\end{array}$ & $\mathrm{XRF} ;-; \mathrm{S}$ & $\begin{array}{l}\text { Portable XRF instrument used to analyse } \\
\text { pottery from four different sites. Data analysis } \\
\text { using PCA with Varimax rotation demonstrated } \\
\text { that much of the pottery was produced locally. } \\
\text { For some sites the finer samples were } \\
\text { imported. However, other sites produced both } \\
\text { fine and less complicated samples. }\end{array}$ & 256 \\
\hline $\begin{array}{l}\text { Various (38) } \\
\text { including REEs }\end{array}$ & $\begin{array}{l}\text { Sherds of Chinese } \\
\text { late Ming export } \\
\text { blue and white } \\
\text { porcelain. }\end{array}$ & MS; ICP; L & $\begin{array}{l}\text { Samples (25) had the glaze ground off and } \\
\text { were then pulverized forming a powder. An } \\
\text { acid dissolution then occurred (full details } \\
\text { given) prior to ICP-MS analysis. Three } \\
\text { Chinese CRMs used for validation (andesite, } \\
\text { basalt and shale). Provenance of the samples } \\
\text { was elucidated thanks to PCA interrogation } \\
\text { of the analytical data. }\end{array}$ & 257 \\
\hline Various (12) & $\begin{array}{l}\text { White clay ceramic } \\
\text { tiles from Veliki } \\
\text { Preslav }\end{array}$ & $\begin{array}{l}\text { OES; LIBS; S, XRF;-; S, } \\
\text { XRD }\end{array}$ & $\begin{array}{l}\text { Q-switched Nd:YAG laser operating at } 1.06 \\
\mu \text { m and with a pulse duration of } 10 \mathrm{~ns} \text { used } \\
\text { during LIBS analyses. Emitted light } \\
\text { transported through a } 10 \mu \mathrm{m} \text { fibre optic to an } \\
\text { Echelle spectrometer and intensified CCD } \\
\text { detector operating between } 220 \text { and } 850 \mathrm{~nm} \text {. } \\
\text { Laser energy of } 6 \mathrm{~mJ} \text { used since this did not } \\
\text { saturate the camera and led to no self- } \\
\text { absorption. } 40 \text { laser pulses were used to } \\
\text { obtain a good signal to noise ratio. EDXRF } \\
\text { spectrometer also used without sample } \\
\text { treatment. Elements in the range } 0 \text { to } 40 \mathrm{keV} \\
\text { monitored. XRD detected mineral } \\
\text { composition. This combined analytical } \\
\text { approach enabled complete chemical } \\
\text { characterization of samples. }\end{array}$ & 258 \\
\hline
\end{tabular}


Table 3 (Contd.)

\begin{tabular}{|c|c|c|c|c|}
\hline Element & Matrix & $\begin{array}{l}\text { Technique; atomization; } \\
\text { presentation }\end{array}$ & Comments & Ref. \\
\hline Various & $\begin{array}{l}\text { Unglazed } \\
\text { earthenware } \\
\text { artefacts }\end{array}$ & LIPS;一; S & $\begin{array}{l}\text { Home-made system developed comprising } \\
\text { six Czerny-Turner monochromators. } \\
\text { Calcium is known to accumulate in the } \\
\text { surface of buried ceramics. The method } \\
\text { developed was capable of depth-profiling Ca. } \\
\text { Discrimination between genuine ancient } \\
\text { ceramics and modern imitations was } \\
\text { therefore enabled. }\end{array}$ & 259 \\
\hline Various & $\begin{array}{l}\text { Heavily-tempered } \\
\text { ceramic pastes }\end{array}$ & MS; ICP; LA & $\begin{array}{l}\text { Samples previously characterized using NAA } \\
\text { were analysed using LA-ICP-MS to determine } \\
\text { if the latter methodology could reproduce } \\
\text { the data for the clay and the temper within } \\
\text { the pottery. The LA-ICP-MS results } \\
\text { demonstrated that the clay fraction could be } \\
\text { isolated and that the data for the clay } \\
\text { fractions were nearly identical to those } \\
\text { obtained using NAA for raw clay samples. } \\
\text { However, LA-ICP-MS could not replace the } \\
\text { more precise data generated using NAA } \\
\text { when the bulk sample was analysed, i.e. both } \\
\text { clay and temper. }\end{array}$ & 260 \\
\hline
\end{tabular}

supporting Mo layer was employed the characteristics of the ablation craters produced in the sample were completely different to those without this layer present. It was further observed that the electron densities from the plasma, calculated using the measured LIBS spectra, were lower by as much as a factor of four when the Mo supporting layer was employed. The effect was considered to result from a matrix interference arising from ionisation of $\mathrm{Na}$ atoms that had diffused into the Mo sub-film. Nevertheless, it was suggested that LIBS could be used to characterise such systems with particular reference to the measurement of $\mathrm{Na}$ content of each layer.

A novel dual laser ablation process had been used to grow epitaxial lead zirconate titanate (PZT) thin films on $\mathrm{SrTiO}_{3}$ and $\mathrm{MgO}$ substrates. One of the problems with existing methods is that the ejection of molten $\mathrm{Pb}$ from the target can lead to particulate-laden films affecting heterostructure growth. In this dual laser ablation approach, pulses from an excimer laser and a carbon dioxide laser were used to overcome $\mathrm{Pb}$ depletion in the material and increase the $\mathrm{Pb}$ content of such films while minimising the formation of particulates and other defects. Intensified CCD imaging and optical emission spectrometric observation of the laser plumes indicated that broader angular expansion and enhanced excitation of the ablated species was achieved using a dual laser as opposed to a single laser ablation process. $^{269}$ The behaviour of laser cooled near-surface Cs atoms close to a silica optical nano-fibre has been investigated using laser induced fluorescence spectroscopy. ${ }^{270}$ Excitation spectra were obtained by observing fluorescence photons through the guided modes. It was found that the Cs atoms exhibited spectra that suggested they were surface bound rather than free. The spectral line shape observed could be influenced by altering conditions at the fibre surface, indicating a possible means of controlling absorption and desorption processes.
A LA-TOF-MS has been described for micron scale imaging and depth profiling of thin metal foils and semiconductor wafers. ${ }^{271}$ The system utilised a Ti:sapphire laser for ablation operating at an output wavelength of $800 \mathrm{~nm}$, generating $75 \mathrm{fs}$ pulses at $1 \mathrm{k} \mathrm{Hz}$. Mass spectra could be acquired by the instrument using pressures ranging from $10^{-8}$ to $0.3 \mathrm{mbar}$ at a rate of $1 \mathrm{kHz}$. Results were reported that demonstrate the feasibility of elemental quantification and depth profiling using the LA-TOF-MS system. The thickness of sub-nanometre metallic layers deposited on silicon wafers has been determined by LA-ICP-MS. ${ }^{272}$ Wafers were coated with Nd layers of $0.5,1,3$, and $6 \mathrm{~nm}$ thickness and covered by a further $10 \mathrm{~nm} \mathrm{Al}$ layer. It was reported that integrated ion signals correlated with layer thickness. Using the ablation of a glass SRM and the Al layer as a reference, sensitivity ratios were obtained to calibrate the instrument response for the thickness measurement. The difference between the prepared thickness and the measured thickness using this strategy was given as less than $10 \%$. In a similar way, laser-induced plasma spectroscopy has been used to determine composition and thickness of protective coatings used in the naval sector. ${ }^{273}$ Finally, a mass spectrometric study has been conducted to seek to understand the surface chemistry of $Y$ thin films used in photo cathodes. ${ }^{274}$ Such films are grown using the pulsed LA technique. The effect of laser energy on the formation of ionic species in the ablation of a Y target was examined by time-integrated and timeresolved MS. Hydride, oxide and nitride species of Y were only observed in the MS spectrum when a laser fluence of $7.6 \mathrm{~J} \mathrm{~cm}^{-2}$ was used, but not at the more moderate setting of $4.5 \mathrm{~J} \mathrm{~cm}^{-2}$. It was also noted that due to deposition of a $\mathrm{Y}$ layer on the internal wall of the vacuum system during the ablation process, a getter effect was observed resulting from absorption of the residual gas. 
3.2.2. Glow discharge and plasma techniques. The direct analysis of thin films and layers continued to be explored using glow discharge sampling for mass spectrometry. For example, a pulsed rf GD-TOF-MS instrument has been used in the characterisation of ultrathin $\mathrm{Si}-\mathrm{Co}$ bilayers deposited on a silicon substrate. ${ }^{275}$ The samples contained an external layer of Si only 30 $\mathrm{nm}$ thick with internal Co layers in the range 1-30 $\mathrm{nm}$ thick. The top layer and the substrate had essentially the same matrix composition (>99.99\% Si). The analysis of such thin materials represents a significant analytical challenge. The purpose of this study was to demonstrate the effectiveness of using the analyte pre-peak pulse time region during the sputtering process to make analytical measurements. In this phase of the cycle, the analyte ion concentrations peak after the initial sputtering caused by each GD pulse. It was anticipated that this approach would improve the resolution of the depth profile analysis and at the same time minimise spectral interferences. The results obtained using this approach were compared with those achieved using TOF-SIMS and the authors reported that the outcome was favourable. Depth profiling analysis of $\mathrm{Bi}-\mathrm{Te}$ and $\mathrm{Sb}-\mathrm{Te}$ thermoelectric layers deposited on $\mathrm{Au}$ and $\mathrm{Cr}$ coated silicon wafers has been achieved using MS methods. ${ }^{276}$ The performance of a pulsed rf GD or plasma profiling TOF-SIMS instrument was assessed in comparison with that of a conventional SIMS system. The depth resolution of both systems was similar but the rf GDTOF-SIMS method was faster due to higher sputtering rates. The latter system was also able to achieve quantitative analysis if the stoichiometry of one sample was determined by ICP-OES and SEM-EDXRF. The standard deviations achieved for the same sample ranged from 1.1 to $1.9 \%$ for the $\mathrm{Bi}_{2} \mathrm{Te}_{3}$ layer and $3.6 \%$ from sample to sample. The equivalent precision data obtained for the $\mathrm{Sb}_{2} \mathrm{Te}_{3}$ layer were in the range $1.7-42 \%$ for a single sample and $5.3 \%$ from sample to sample.

The utility of a pulsed glow discharge in a Grimm-type source for the OES analysis of thin layered and thermally fragile samples has been explored by a group in Dresden. ${ }^{277}$ The selection of key operating conditions such as duty cycle, frequency and pulse length to achieve optimal sputtering and crater shape were investigated. It was noted that while operation in dc or rf modes did not greatly alter crater shape and thermal effects, the choice of pulse length had a significant effect and was an important parameter in optimising the resolution of depth profiles. The effect of oxygen on glow discharge emission spectra resulting from Fe samples covered with an oxide layer has been investigated. ${ }^{278}$ A sample of Calamine was analysed by GD-OES in a pure argon plasma and time resolved spectra and depth profile information acquired. A comparison was made of the effect of oxygen derived from this type of sample with an oxide layer and that from controlled addition of oxygen to the argon carrier gas for the analysis of a pure Fe sample. It was reported that analytically the effect of oxygen from the layered sample was similar in magnitude to that from sputtering in argon with $0.05 \% \mathrm{v} / \mathrm{v}$ oxygen in the carrier gas. The application of GD-OES to the examination of organic molecular monolayers adsorbed onto $\mathrm{Ag}, \mathrm{Au}$ and $\mathrm{Cu}$ surfaces has been described. ${ }^{279}$ A radiofrequency GD was used to study the adsorption of thiourea on metal surfaces and on metal centred (Co) molecular wires of $4-5 \mathrm{~nm}$ in length. The latter molecules were adsorbed on a gold substrate. The depth profile analysis spectrum for the $\mathrm{Cu}$ surface showed a $\mathrm{N}$ peak followed by a $\mathrm{S}$ peak, confirming that thiourea was absorbed onto the metal surface via a $\mathrm{S}$ atom interaction. The positions of the $\mathrm{N}$ and $\mathrm{S}$ peaks were not consistent for $\mathrm{Ag}$ and $\mathrm{Au}$ surfaces, and either overlapped or were in the reverse order in comparison with that for $\mathrm{Cu}$. For the metal centred molecular wires, the $\mathrm{S}$ peak appeared prior to the Co peak with respect to sputtering time. The sputtering time for thiourea and wire molecules were similar although the lengths of the molecules differed by more than an order of magnitude. It was concluded that for these systems it was not possible to correlate the appearance of depth profile peak sequence with molecular orientation on the surface.

A real time process control system based on emission spectrometry has been described for use in a chemical vapour deposition process for the creation of microcrystalline $\mathrm{Si}$ films. ${ }^{280}$ The operation of the system was based on the observation of optical emission from a very high frequency plasma employed to enhance the deposition process. The spectral intensity data were used in a closed loop feedback system. By modulating the VHF power and silane dilution in the gas phase, it was found possible to improve microcrystalline Si film growth. The OES system was shown to be able to stabilise the $\mathrm{H} / \mathrm{SiH}$ intensity ratio to within $5 \%$ during the deposition process. The approach achieved higher film growth rate while at the same time consuming less power and silane gas than the method without process control.

\subsection{Glasses}

Publications in the review period concerning the analysis of glasses fell into two categories: those that were concerned with the characterisation of new functional materials; and those that were focused on identifying the origin or provenance of glasses. Interest in the examination of glasses associated with cultural heritage remains strong, and the approaches described in these studies may have particular relevance to forensic or commercial investigation the source of glasses.

A review of techniques used in the assessment of materials for glass-making may be of general interest. ${ }^{281}$ This article summarised approaches to sampling for subsequent instrumental analysis such as XRF and ICP-OES, and classical wet chemical analytical methods. A review of X-ray synchrotron micro-beam techniques that can be used in supporting heritage conservation has been published. ${ }^{282}$ The article focused on a description of the principles of XRF, XRD and X-ray absorption, the information provided by each technique and applications relating to glasses, marble and timbers. The application of LA-ICP-MS for the quantitative multi-element analysis of ancient glasses has been the subject of a recent review. ${ }^{283}$ The article explored the use of reference materials and standards, response factors, fractionation and matrix effects, laser sampling, data reduction strategies, quantification approaches and analytical performance (including bias and reproducibility) with specific reference to the use of LA-ICP-MS in this field. 
The multi-technique characterisation of new silicate reference glasses for use in microanalysis has been described. ${ }^{284}$ The silicate reference glasses BAM-S005-A and BAM-S005-B were produced by the Federal Institute for Materials Research and Testing, Germany. The homogeneity of the materials was studied using EPMA, LA-ICP-MS and SIMS. It was reported that while most elements were homogenously distributed at the micrometer sampling scale, some trace elements (Cl, Cr, Cs, Mo and $\mathrm{Ni}$ ) were not. The certificate values for bulk composition were also confirmed using EPMA and LA-ICP-MS and data for a further 22 trace elements were reported. However, for 4 trace elements (Ba, Ce, $\mathrm{Pb}$ and $\mathrm{Sr}$ ) the LA-ICP-MS results were not in agreement with certified values, but no reasons for the discrepancy were given. It was concluded overall that the reference materials could be used in many micro-analytical applications.

The surface chemistry of zinc polyphosphate glasses has been investigated using a combination of XPS, TOF-SIMS and FTIR. ${ }^{285}$ Samples ranging from zinc metaphosphate to zinc orthophosphate were studied with a view to identifying the chain lengths of these materials. It was reported that XPS could identify chain length by measurement of the integrated intensity ratio of the bridging $\mathrm{P}-\mathrm{O}-\mathrm{P}$ and non-bridging $\mathrm{P}=\mathrm{O}$ and $\mathrm{P}-\mathrm{O}-\mathrm{M}$ oxygen peaks used for fitting the oxygen 1s signal while monitoring the $\mathrm{P} 2 \mathrm{p}_{3 / 2}$ signal shift to lower binding energies and the auger parameter to higher values as the $\mathrm{Zn}$ content was increased. By comparing the intensities of characteristic phosphate sputter fragments it was found possible to use TOF-SIMS spectra to distinguish between chain lengths. Further investigation by FTIR confirmed that chain lengths became shorter as the zinc content was increased.

Functional optical glass materials are now being produced to act as planar waveguides structures. In one such system, a telluride glass of the generic type $\mathrm{TeO}_{2}-\mathrm{WO}_{3}-\mathrm{La}_{2} \mathrm{O}_{3}-\mathrm{Na}_{2} \mathrm{O}$ (TWLN) has been doped with $\mathrm{Ag}$ layers and the elemental concentration profile determined by SIMS and RBS. ${ }^{286}$ A solid state field assisted diffusion process was used to prepare the materials. It was reported that the experimental data obtained for the Ag concentration profile using SIMS and RBS were in good agreement with those calculated using a theoretical model. The use of arsenic-sulfoselenide glasses in the fabrication of optical components in light transmitting optics and fibres is well established. However when XRF is used to quantify the composition of such materials, it is found that the X-ray signal is a non-monotonic function of As and Se concentrations and that use of model based corrections can give rise to errors in the analysis. ${ }^{287}$ Consequently, in this work a number of reference materials were used as samples in order to model the problem. It was reported that the use of a fundamental parameter approach yielded results that did not reduce the error in comparison with those from empirical calibration. However, it was found that by selective use of reference materials as standards, the error in the determination could be reduced by ensuring a monotonic relationship between the relative X-ray intensity and the target element concentration.

The structure and properties of a novel glass containing $\mathrm{SeO}_{2}$, $\mathrm{TeO}_{2} \mathrm{MoO}_{3}$ and $\mathrm{La}_{2} \mathrm{O}_{3}$ were investigated using a range of techniques, including atomic spectrometry. ${ }^{288}$ Thus LA-ICP-MS was used to assess the bulk chemical composition revealing a $17: 50: 32: 1$ ratio of the respective oxide components. The XPS results indicated that the main unit structures consisted of $\mathrm{TeO}_{3}, \mathrm{TeO}_{4}, \mathrm{SeO}_{3}$ and $\mathrm{Mo}_{2} \mathrm{O}_{8}$ and that this led to the creation of an amorphous network consisting of mixed bridging bonds.

The determination of $\mathrm{Pb}$ in glass containers used for food and beverages has been the subject of investigation for many years because of the possible risk to health. Techniques such as ICPOES, AAS and WD-XRF have been routinely used for the measurement of $\mathrm{Pb}$ in applications of this type. However, a rapid and simple procedure based on the use of a portable EDXRF instrument has been now been described. ${ }^{289}$ The portable instrument was calibrated using CRMs and reference samples previously characterised by classical analytical chemistry methods. Samples, (either flat polished or the unprepared original container surface) of a variety of types of soda-limesilica glass (white, half white, emerald green, yellow green and amber) were analysed quantitatively using this approach and the results compared to those obtained from a conventional WDXRF method. Results for $\mathrm{Pb}$ and other elements of interest (As, Cr, Fe and $\mathrm{Mn}$ ) were found to be satisfactory in terms of accuracy and precision. The instrument sensitivity was sufficient to detect analytes at the levels present in the samples and a LOD of $30 \mathrm{mg} \mathrm{kg}^{-1}$ for $\mathrm{PbO}$ was reported.

3.3.1. Laser-based techniques. The application of laserbased sampling techniques to the forensic analysis of glass continues to be an area of research interest. ${ }^{290}$ Thus the analytical performance of LA-ICP-OES has been compared to LA-ICP-MS for the determination of 9 elements in 41 glass samples. Sampling for both systems was carried out using a Q-switched Nd:YAG laser operating at $213 \mathrm{~nm}$. The ICP instrument utilised an echelle polychromator with tow segmented array CCDs for uv and visible spectrum measurement, and the plasma was viewed in an axial configuration. It was noted, unsurprisingly, that larger sample masses sizes (400-500 ng) were required for ICP-OES measurement and that the ablation spot sizes were also larger $(100 \mu \mathrm{m})$ than required for ICP-MS analysis (40-60 $\mu \mathrm{m})$. A FGS 1 reference material (BKA Forensic Science Unit, Wiesbaden, Germany) was used as the single point calibration standard for the method and NIST SRM 1831 run every two hours to check for instrumental drift. Limits of detection for the ICP-OES method ranged from $0.2 \mu \mathrm{g} \mathrm{g}^{-1}$ for $\mathrm{Sr}$ to $13.1 \mu \mathrm{g} \mathrm{g}^{-1}$ for Fe. A much higher LOD of $559 \mu \mathrm{g} \mathrm{g}^{-1}$ was reported for $\mathrm{Ca}$ due to the use of the less sensitive $315.88 \mathrm{~nm}$ line for the measurement of percentage levels of that element in the glass. Bias and precision for the method were both estimated as $<10 \%$. The LA-ICP-OES system was found to perform similarly to LA-ICP-MS in differentiating between vehicle glass fragments from different sources. A study comparing the use of refractive index and LA-ICP-MS for the forensic analysis of glass particles may also be of interest to those working in the field. ${ }^{291}$

The classification and discrimination of automotive glass of relevance in New Zealand has been the subject of a recent study. ${ }^{\mathbf{2 9 2}}$ The elemental composition of 243 samples of automotive glass was assessed using direct sampling with LA-ICP-MS. In most cases there was no noticeable variation in the spatial 
distribution of elements in the glass panes. However, the $\mathrm{Pb}$ content was found to be the most variable spatially. It was possible using the analytical results to distinguish relatively easily between glasses originating in the Northern hemisphere and from Australian sources. The latter class of samples showed much less variation within the group. However, using both elemental composition and refractive index, a method was proposed that was able to distinguish 170 out of 204 samples (84\%). When Australian samples were removed, the discrimination of the method increased to $95 \%$ (148 out of 156 samples).

The determination of uranium isotope content of micron-scale doped glass fragments has been the subject of a study using ICPMS. ${ }^{293}$ The operating parameters of a ns LA system coupled to a MC-ICP-MS were investigated. The secondary electron multiplier, with which ${ }^{236} \mathrm{U}$ was measured, had a deceleration filter installed to eliminate ions with low kinetic energies in order to improve abundance sensitivity. Certified reference materials (CRM U500 (New Brunswick Laboratory, US Department of Energy Washington DC USA) and IRMM 187 (European Commission, JRC, Geel Belgium)) were evaluated for the purposes of external calibration correction. In order to allow convenient switching between solid and liquid sample analysis modes, a membrane desolvation system was employed in parallel with the LA cell. No liquid sample was aspirated using the LA process. A natural U solution (IRMM-184) was used for the determination of the $\mathrm{UH}^{+}: \mathrm{U}^{+}$hydride ratio when introducing liquid standards and was diluted as required for determinations at low concentration levels (i.e. $<10 \mathrm{ng} \mathrm{g}^{-1}$ ). The effect of Ar and He as ablation carrier gases on accuracy and precision was investigated but it was reported that, contrary to the finding of other workers, there was no difference in analytical performance arising from the use of He. It was reported that the application of a deceleration filter was proven to be a prerequisite for the accurate determination of very low ${ }^{236} \mathrm{U} /{ }^{238} \mathrm{U}$ isotope ratios (i.e. $10^{-5}$ ). Results obtained were in good agreement with certified values and detailed uncertainty budgets for the methodology were produced. The multi-technique characterisation of nuclear waste glass materials and related corrosion and weathering effects may also be of interest in this context. ${ }^{294,295}$

Element fractionation in laser ablation inductively coupled plasma mass spectrometry applications remains a problematic issue. This effect has been studied with reference to the response of semi-volatile metals in the analysis if silicate glasses. ${ }^{296}$ In a novel approach intended to address element fractionation, the design of a two volume ablation chamber was described and its influence on analytical performance for the determination of $\mathrm{Cd}, \mathrm{Cu}, \mathrm{Mo}, \mathrm{Pb}, \mathrm{Sn}$ and $\mathrm{Zn}$ compared to that of a conventional single volume chamber. For the single ablation chamber, the differential response of these elements was evaluated relative to ${ }^{43} \mathrm{Ca}$. The variability of fractionation was reported to be related not to the interaction between laser pulse and sample but to the local He carrier gas velocity at the position of the analysis. This was found to affect not only volatile elements but also others (Ag, B, Co, Cu, In, Mo, Pb, Sb, Sn, W, Zn). These problems were not observed when a two volume ablation chamber was used, presumably because the He flow rate across the chamber remained more uniform. The response precision for normalised ratios of volatile metals using this method was given as $<10 \%$. A further investigation of matrix effects in LA-ICP-MS has been published recently. ${ }^{297}$ This study involved an assessment of whether or not glass transparency affected ablation behaviour. In order to test this proposition, transparent NIST, BAM and USGS reference glasses were analysed by LA-ICP-MS using a Nd:YAG laser with an output wavelength at $193 \mathrm{~nm}$. The samples were selected because they had good elemental homogeneity at the $\mu \mathrm{m}$ scale. It was reported that the observed fractionation of refractory and volatile lithophile elements (e.g., Ba, Rb, Sr) was minimal and not dependent on glass transparency. However, the material transparency was a significant factor in influencing fractionation for chalcophile and siderophile elements (such as $\mathrm{Pb}$ and $\mathrm{Zn}$ ). The fractionation factors in the latter case were found to vary between 0.7 and 1.0 and were also dependent on laser ablation spot size. It was noted that for volatile chalcophile and siderophile elements, mass-load matrix effects accounted for up to $10 \%$ of the signal when the mass load varied by a factor of 2.4. Relative sensitivity factors were calculated for calibration purposes and were consistent with uncertainty limits for the lithophile content of transparent and opaque glasses and were within 5$10 \%$ for the other element classes. The LA-ICP-MS results for the concentration of 27 trace elements (in the ranges 1 and 500 $\mu \mathrm{g}^{-1}$ ) in the reference materials were provided and uncertainties at the 95\% confidence level varied between 1and $4 \%$ and 7 and $10 \%$ respectively for the two ranges.

An investigation of matrix effects in the LA-ICP-MS determination of major and minor elements in anhydrous silicate samples has been described. ${ }^{298}$ An ultraviolet (200 nm) femtosecond LA sampling unit was coupled to a ICP-MS instrument employing a sector field. It was reported that the use of this laser resulted in a reduction in matrix effects by up to $50 \%$ of those found when using a $193 \mathrm{~nm}$ excimer laser for the same purpose. This improvement was attributed to the suppression of element fractionation effects caused by sample melting. However, it was noted that for those elements with high first ionisation energy response sensitivity was enhanced for basalt relative to silicarich aerosols. A simulation using the Saha equation showed that the higher thermal conductivity of the basalt aerosol in the ICP enhanced ionisation, confirming the basis of this effect. Using a single basalt glass as a calibration standard, results obtained using the method were within $5 \%$ of accepted values and it was reported that this approach was suitable for application to the analysis of a range of silicate materials.

A novel femtosecond fibre laser has been described for use in portable laser induced breakdown spectroscopy. ${ }^{299}$ The system comprised a $1013 \mathrm{~nm}$ fs fibre laser and an intensified CCD detection system. The atom and ion emission line spectra produced during the qualitative analysis of glass, metal and semiconductor materials using this instrument were characterised. Time resolved spectra were recorded during the development of the laser induced plasma. It was reported that the intensified CCD used provided a 20 -fold improvement in SNR compared with a non-gated, non-intensified detector. It was commented that the system was portable and potentially field 
deployable. A portable laser sampling device has been described that allows the elemental fingerprinting of solid samples outside the laboratory environment. ${ }^{52}$ This system utilised a diode pumped solid state laser to sample materials in situ onto membrane filters. These filters were subsequently subjected to analysis by LA-ICP-MS. Glass and gold reference materials were used to assess the analytical performance of the method. The LODs achieved were between 0.01 and $1 \mu \mathrm{g} \mathrm{g}^{-1}$ and accuracies of better than $20 \%$ were reported for most elements.

3.3.2. Other techniques. A new Grimm-type ion source designed for time-of-flight mass spectrometry for the direct analysis of glasses has been described. ${ }^{300}$ This rf ms-pulsed GD was interfaced to a TOF-SIMS and its performance compared with that of a previous source design. The new 'UNIOVI' source chamber thickness was less than half that of the original design $(7 \mathrm{~mm}$ c.f. $15.5 \mathrm{~mm})$ and the flow tube used in the earlier system was removed. The source was operated with a pulse period of 4 ms and a duty cycle of $50 \%$. The effect of experimental conditions, including pressure and applied power on signal shapes along the pulse, were also investigated and a comparison of the analytical performance of the two sources was made for this application. It was reported that the new source induced less thermal stress on the glass samples and that as a result it was possible to use higher power settings than possible previously (up to $130 \mathrm{~W}$ c.f. $60 \mathrm{~W}$ ). Since glass breakdown was avoided with the new source at higher powers, better crater shapes were also obtained. Both sources provided comparable analytical results for the analysis of glasses. However, LODs were found to be about an order of magnitude better for the system employing the new source for the 18 elements investigated.

Prefilled glass syringes are now widely used in the pharmaceutical industry and are subject to restrictions on elemental impurity limits. The leaching of elements from glasses used in commercial syringe products has been investigated using sector field ICP-MS. ${ }^{301}$ The system was applied to the determination of $\mathrm{Al}, \mathrm{Na}, \mathrm{Si}, \mathrm{W}$ and 16 other elements listed in draft chapter 232 of the US Pharmacopeial Convention, selected on the grounds of toxicity. Unsurprisingly, $\mathrm{Na}$ (up to $1200 \mu \mathrm{g} \mathrm{\textrm {L } ^ { - 1 }}$ ) and Si (up to $4500 \mu \mathrm{g} \mathrm{L}{ }^{-1}$ ) were found in all leachate solutions. However, in one case, $\mathrm{Al}$, As and $\mathrm{W}$ were found in leachates at average concentrations of $400,0.55$ and $230 \mu \mathrm{g} \mathrm{L} \mathrm{L}^{-1}$ respectively. These levels were nevertheless still within the guideline limit concentrations.

The provenance and origin of glasses containing ${ }^{137}$ Cs is a matter of concern as the radioisotope is widely used in sealed radioactive sources. Thus, a method has been developed to separate and quantify $\mathrm{Cs}$ and Ba from glasses with high lanthanide content using ICP-MS. ${ }^{302}$ The age of the ${ }^{137} \mathrm{Cs}$ present is dependent on the date of separation from its radiochemical series daughter ${ }^{137} \mathrm{Ba}$, hence, it was necessary to develop a method to determine the concentration of both isotopes in order to estimate the age of the material. Samples of glass and high purity silica (as a control) were dissolved in hot concentrated nitric and hydrofluoric acids followed by fluoride compensation with boric acid and the evolution of excess boric acid as methyl borate. The material was then converted to nitrate species using $3 \mathrm{M}$ nitric acid before separation by gas pressurised extraction chromatography on an Erichrom Sr resin column. The Cs was eluted form the column using $3 \mathrm{M}$ nitric acid and the Ba was subsequently removed using $1 \%$ acetic acid. Following analysis by ICP-MS, it was reported that quantitative recovery was achieved for both Cs and Ba in both the lanthanide glass matrix and the control silica material.

The application of particle induced gamma-ray emission (PIGE) has been described for the measurement of fluorine in barium borosilicate glasses. ${ }^{303}$ Thick pellet samples were prepared in a cellulose matrix and subjected to a $4 \mathrm{MeV}$ proton beam form the folded tandem ion accelerator at BARC, Mumbai. Beam current variation was monitored by RBS in order to normalise the count rate of interest and by addition of an internal standard with good PIGE sensitivity. The method was validated using synthetic standards.

3.3.3. Analysis of archaeological, cultural heritage and art objects. The bulk of the reports received in the period under review relating to the analysis of cultural heritage materials have concentrated on the application of established techniques rather than to particular advances of interest to the wider analytical community. However, examples of the outcomes of such studies are cited here as a summary of the impact that atomic spectrometry techniques are now having in this field.

The application of EPMA and LA-ICP-MS to the analysis of late Roman window and vessel glasses from the Great Temple of Petra has been described. ${ }^{304}$ Forty seven samples were analysed for major and minor elements by EPMS and a subset of twenty nine samples for trace components by LA-ICP-MS. It was reported that six compositional groups were identified on the basis of the presence of decolouring agents indicating evidence of recycling The effect of laser treatment on the morphology of Roman glass of Serbian origin has been investigated. ${ }^{305}$ Three different laser systems (TEA $\mathrm{CO}_{2}, 10.6 \mu \mathrm{m}, 100 \mathrm{~ns}$ pulse; Nd:YAG, 1064 and $532 \mathrm{~nm}, 150$ ps pulse; and Ruby $694 \mathrm{~nm}, 30$ ns pulse) were used to ablate materials in ambient air. It was noted that under the moderate energy densities used (15 to $30 \mathrm{~J}$ $\mathrm{cm}^{-2}$ ) significant changes in sample morphology were produced. It was reported that the pulsed $\mathrm{CO}_{2}$ laser could be optimally used for surface cleaning of the materials at a fluence of $2 \mathrm{~J} \mathrm{~cm}^{-2}$.

A novel application of ETV-ICP-OES to the direct characterisation of archaeological glasses has been described. ${ }^{306}$ Samples of coloured Roman window glass from the 5th century and medieval glass bracelets from the 11th and 12th centuries excavated in West Bulgaria were prepared by grinding. These powders were then analysed directly, employing trifluoromethane as a modifier to enhance evaporation and transport. Calibration was achieved using dried aqueous standards and a range of glass and other CRMs. Major, minor and trace elements were determined in the samples using the procedure without any evidence of matrix effects. It was reported that characteristics such as the type of glass, the fluxing agents used and the colouring materials could be identified from these results.

The use of strontium and neodymium isotope ratios to provide evidence of provenance of raw materials used in the production of archaeological glass has also been the subject of an investigation involving multi-collector ICP-MS. ${ }^{307}$ In order to achieve 
reliable results, effects due to mass discrimination were eliminated by the isolation of the target element from the matrix. Powdered glass samples (100 mg), were pre-digested in $3 \mathrm{ml}$ $22 \mathrm{M} \mathrm{HF}$ and $1 \mathrm{ml}$ of $14 \mathrm{M} \mathrm{HNO}_{3}$ in a PFA screw top beaker on a hot plate at $110{ }^{\circ} \mathrm{C}$ overnight. Following evaporation to dryness, the residue was taken up in $4 \mathrm{ml}$ of aqua regia and evaporated to dryness again before solubilisation in $7 \mathrm{M} \mathrm{HNO}_{3}$ prior to separation. The Nd was separated from the sample matrix using two chromatographic resin packed micro columns. The first of these utilised chromatographic micro columns packed with TRU-Spec resin $(600 \mu \mathrm{l})$ and was used for primary matrix separation. Following washing with $4 \mathrm{ml}$ of $2 \mathrm{M} \mathrm{HNO}_{3}$, the first column was connected to a second Ln-Spec resin column capacity $(1.5 \mathrm{ml})$ for secondary extraction from LREEs and $\mathrm{Nd}$ eluted using $0.25 \mathrm{M} \mathrm{HCl}$. A separate parallel procedure for the separation of $\mathrm{Sr}$ (based on the use of Sr-spec resin and elution with $5.5 \mathrm{ml}$ of $0.05 \mathrm{M} \mathrm{HNO}_{3}$ ) was also described. Isotope ratios for $\mathrm{Nd}$ and $\mathrm{Sr}$ were measured using a multi-collector ICP-MS instrument. The intensities for ${ }^{147} \mathrm{Sm}$ and ${ }^{149} \mathrm{Sm}$ were measured to correct for isobaric overlaps from ${ }^{144} \mathrm{Sm}{ }^{148} \mathrm{Sm}$ and ${ }^{150} \mathrm{Sm}$ on the corresponding $\mathrm{Nd}$ isotopes. The use of elemental and isotopic analysis to characterise natron glass from the Eastern Roman Empire from three archaeological sites (Petra and Barsinia, both in Jordan, and Gonio, in Georgia) may also be of interest in this context. ${ }^{308}$ The provenance of 14th and 15th century English forest glasses has been investigated. ${ }^{309}$ For a subset of five of these samples, TIMS was applied to the determination of $\mathrm{Sr}$ and $\mathrm{Nd}$ isotope ratios to differentiate the glasses and to identify production sites within each region.

Proton induced $X$-ray emission and proton induced gamma emission techniques have been used extensively to study the provenance of archaeological glasses. Thus rare enamelled glass samples from 15th to 17th century Venice have been examined non-destructively using PIXE-PIGE techniques. ${ }^{\mathbf{3 1 0}}$ Eleven glass pieces taken from the Louvre collection were analysed and results, allowing the identification of objects that were of genuine Venetian renaissance provenance. Other similar examples of the use of PIXE-PXE reported in the period under review include applications to the examination of medieval glass fragments, selected from the archaeological museum in Savona, Italy; ${ }^{311}$ Bulgarian archaeological glasses ${ }^{312}$ and medieval glass bead samples from Slovenia; ${ }^{313}$ andThailand. ${ }^{314}$ Similarly, Roman glass from South West Iberia has been examined using a combination of synchrotron micro-XRF, PIXE-PIGE and SEM with an energy dispersive detector. ${ }^{\mathbf{3 1 5}}$

The application of portable X-ray fluorescence spectrometry to the on-site analysis of ancient glass vessel fragments has been described. ${ }^{316}$ Samples of Thai glass dating from the 7 th to 9 th centuries were analysed directly using a portable EDXRF spectrometer modified with a polymer window fitted to the silicon drift detector which allowed the detection of light elements. The origin of the glass and possible links to similar materials at sites in Egypt was discussed. In a related publication, the use of cobalt blue colorant in glasses of the New Kingdom of Egypt was investigated. ${ }^{317}$ Glass beads of Silk route origin, have been characterised using a high resolution portable XRF spectrometer. ${ }^{318}$ The quantitative chemical composition data, when combined with archaeological information, provided an insight into the source of these glasses which dated back to the Tang Dynasty. A handheld XRF spectrometer has been successfully applied to distinguish panes of 17th century high lime, low alkali glasses in situ at Christ Church Cathedral, Oxford, England. ${ }^{319}$ The system was also applied, unsuccessfully, to the characterisation of Roman glasses and the reasons for this difference in outcome were explored. In the same vein, the use of X-ray absorption spectrometry and synchrotron excited XRF has been described in application to the characterisation of stained glass samples from excavation of a Cathedral site on Paderborn, Germany. ${ }^{320}$ The XRF results obtained were compared to those achieved using LA-ICP-MS, while XANES was used for chemical speciation.

\subsection{Catalysts}

The field of catalysis continues to be hugely diverse in both the materials used and their application. Consequently, a wide variety of papers were published during the review period. However, many of these provided limited detail of characterization by atomic spectrometric techniques, instead focusing on preparation methods and reaction activity. For the purpose of this review, only papers that include sufficient detail of advances in or application of atomic spectrometric techniques were included.

3.4.1. Surface characterisation. With the majority of reactions occurring at the catalyst surface, techniques that examine the physical and chemical properties of the catalyst surface and its interactions with reactants and poisons are becoming increasingly popular. Chenakin et al. ${ }^{321}$ discussed the surface characterisation of aluminovanadate oxide catalyst precursors using a combination of XPS and TOF-SIMS. Samples were prepared by coprecipitation of $\mathrm{Al}\left(\mathrm{NO}_{3}\right)_{3} \cdot 9 \mathrm{H}_{2} \mathrm{O}$ and $\mathrm{NH}_{4} \mathrm{VO}_{3}$ from aqueous solution at varying $\mathrm{pH}$ followed by drying and calcining. Powdered samples were pressed into indium film to form a uniform layer and loaded into a combined XPS-TOF-SIMS instrument at $8.1 \times 10^{-10}$ mbar base pressure. For XPS, nonmonochromatic $\mathrm{Mg} \mathrm{K}$ radiation of $150 \mathrm{~W}$ operating power was employed. The static TOF-SIMS analysis was carried out using a pulsing beam of $\mathrm{Ar}^{+}$ions and positive secondary ions of mass up to $400 \mathrm{amu}$ were extracted at $1400 \mathrm{~V}$. Changes in the binding energy separations $(\Delta \mathrm{BE})$ between the $\mathrm{O} 1 \mathrm{~s}$ and $\mathrm{Al} 2 \mathrm{p}$ peaks, and the $\mathrm{O} 1 \mathrm{~s}$ and $\mathrm{V} 2 \mathrm{p}_{3 / 2}$ peaks were used to study the surface chemical environment, as this parameter was independent of surface charge. The surface $\mathrm{Al}$ of dried samples had an increasing tendency to form hydroxide-oxohydroxides at higher $\mathrm{pH}$. The major oxidation state of $\mathrm{V}$ was +5 , although an appreciable amount of $\mathrm{V}^{4+}$ species was also present together with hydroxylated $\mathrm{V}$. After calcination the relative fraction of reduced $\mathrm{V}^{4+}$ species increased, the extent of hydroxylation of both $\mathrm{Al}$ and $\mathrm{V}$ decreased and the $\mathrm{Al}-\mathrm{O}$ and $\mathrm{V}-\mathrm{O}$ bond strengths changed, giving rise to a modification of the valence band electronic structure. Comparison of SIMS peak ratios showed that a number of interesting processes occurred. The $\mathrm{V}^{+} / \mathrm{Al}^{+}$ratio indicated that the surface $\mathrm{V}$ concentration of dried catalysts was depleted compared with that of the bulk, with enrichment of $\mathrm{V}$ 
at the surface upon calcination. With increasing $\mathrm{pH}$, formation of aluminium hydroxides-oxohydroxide occurred in preference to vanadium hydroxide, indicated by the $\mathrm{AlOH}^{+} / \mathrm{VOH}^{+}$ratio. This trend was also seen for the $\mathrm{VO}^{+} / \mathrm{V}^{+}$peak ratio indicating a surface of aluminium hydroxide with dispersed vanadium species. As a consequence of these chemical and structural alterations, the catalytic activity of calcined aluminovanadate oxide for propane oxidative dehydrogenation increased.

The powerful combination of XPS and TOF-SIMS was also utilized by Wenmu Li $e t$ al. ${ }^{322}$ to characterize the active sites of pyrolysed iron-based oxygen reduction reaction catalysts. By poisoning the active sites with cyanide, the group were able to elucidate the Fe configuration from the $\mathrm{N} 1 \mathrm{~s}$ and Fe 2p XPS spectra. Further investigation with TOF-SIMS mapping in both positive and negative polarity gave evidence of the same spatial location of species originating from $\mathrm{Fe}$ and $\mathrm{CN}^{-}$.

Nano-sized magnesia-zirconia catalysts, for the reduction of carbonyl compounds under mild conditions were characterized by TOF-SIMS. ${ }^{323}$ Samples were presented to the instrument pressed into indium foil and secondary ion spectra collected in both positive and negative polarity using $\mathrm{a} \mathrm{Ga}^{+}$primary ion beam. In positive mode, the isotopic peaks of the main components and their oxy-hydrated compounds, $\mathrm{Mg}^{+}, \mathrm{MgO}^{+}$, $\mathrm{MgOH}^{+}, \mathrm{Zr}^{+}, \mathrm{ZrH}^{+}, \mathrm{ZrO}^{+}, \mathrm{ZrOH}^{+}, \mathrm{ZrO}_{2}{ }^{+}$and $\mathrm{ZrO}_{2} \mathrm{H}^{+}$, were found together with common surface contaminants $\left(\mathrm{Al}^{+}, \mathrm{Ca}^{+}, \mathrm{K}^{+}, \mathrm{Na}^{+}\right)$. The negative spectra contained the isotopic peaks of zirconium oxides and hydroxides. Repeated sputtering at a single site resulted in a decrease in the $\mathrm{Mg}-\mathrm{Zr}$ ratio, indicating that the particles consisted of a zirconia core covered with a magnesia shell. The techniques of XRD and TEM were used to determine the crystal phase and particle size. The "depth profiling" ability of SIMS was also utilized by Henry et al..$^{324}$ to determine $\mathrm{Al}$ gradients across synthesized ZSM- 5 zeolite crystals produced for $C_{4}$ olefin conversion. An $\mathrm{Ar}^{+}$primary ion beam was used to sputter an area $200 \times 200 \mu \mathrm{m}$. Resulting $\mathrm{Si}-\mathrm{Al}$ ratio profiles revealed aluminium depletion at the crystal surface with a gradient from $\sim 140 \mathrm{Si} / \mathrm{Al}$ at the surface to $\sim 20 \mathrm{Si} / \mathrm{Al}$ in the bulk.

Mechanical mixing of Ni-activated carbon catalyst with $R h-\gamma$ $\mathrm{Al}_{2} \mathrm{O}_{3}$ was shown to have a significant catalytic synergy in the methanation of $\mathrm{CO}_{2}{ }^{325}$ Static TOF-SIMS was used to illustrate that no chemical mixing or formation of new structures occurred when the materials were mixed. Both the separate catalysts and a mixture of the two were investigated using pulsed $\mathrm{Ga}^{+}$ions over a $120 \times 120 \mu \mathrm{m}$ square, after being pressed into a silver conductive tape. Charge effects were compensated using a pulsed electron flood gun and experimental conditions were set to ensure total ion fluence was lower than $10^{12} \mathrm{Ga}^{+}$ $\mathrm{cm}^{-2}$, ensuring static conditions. Positive and negative spectra of the separate catalysts showed characteristic secondary ions of $\mathrm{Rh}$ and Ni for each respective sample. The mixed sample contained both $\mathrm{Rh}$ and Ni characteristic secondary ions but there was no evidence of fragments composed of combined $\mathrm{Ni}$ and $\mathrm{Rh}$ atoms. Further investigation of the mixed sample surface composition using XPS showed that both Rh and Ni reduced to metallic components after activation, although remaining similar in composition to their corresponding separate catalyst, showing no agglomeration or interaction. The increased $\mathrm{CO}_{2}$ methanation was, therefore, explained by the in situ production of hydrogen by the activated $\mathrm{Ni}$ catalyst and migration towards the $\mathrm{Rh}-\gamma-\mathrm{Al}_{2} \mathrm{O}_{3}$ where it could readily react with adsorbed $\mathrm{CO}_{2}$.

3.4.2. Sampling and sample preparation. Primary sampling and chemical sample preparation are the first and often most important stages in atomic spectrometric analysis. However, the majority of catalyst research and development is performed on a small scale and the specimen submitted for analysis is commonly "the bulk" of the material. Many materials can also be prepared by a multitude of acid digestion procedures, be they open vessel or microwave assisted, that have become common practices in analytical laboratories. Therefore, for the purpose of this review, only papers that include sufficient procedural detail or use less common sample preparation techniques were included.

A systematic study of different methods for the decomposition of three-way catalytic converter catalysts, prior to analysis using ICP-OES, was presented by Dyachkova et al. ${ }^{326}$ The paper considered six decomposition procedures, four methods of mineral acid dissolution and two combining high temperature potassium pyrosulfate fusion followed by mineral acid dissolution. The test sample was a combined batch of various branded spent catalysts on ceramic base, ground to a $200 \mu \mathrm{m}$ powder. Complete dissolution of the sample was achieved by dissolving the sample successively with $\mathrm{HF}$ and $\mathrm{H}_{2} \mathrm{SO}_{4}$ followed by fusion of the residue with $\mathrm{K}_{2} \mathrm{~S}_{2} \mathrm{O}_{7}$ and dissolution with aqua regia. However, this resulted in a high salt concentration and significant interference from the matrix components (Al, Ce, Fe, $\mathrm{Zr}$ ) hindering the determination of low PGM concentrations. An alternative procedure of dissolving the sample successively with $\mathrm{H}_{2} \mathrm{SO}_{4}$ and aqua regia eliminated the salt background and also allowed a smaller passage of matrix elements into the analytical solution, whilst maintaining recovery of PGM of interest. The relative standard deviation of the method was $\sim 10 \%$ at concentrations of Pd, Pt and $\mathrm{Rh}$ ranging from 0.01 to $0.3 \%$. A new system for the direct sampling of PGM particulates in motor vehicle exhaust gases that also simultaneously analysed the exhaust gas was developed by Paraskevas et al. ${ }^{327}$ Previous sampling procedures, including acid bubblers or filters following a Dimroth condenser, alter the exhaust gas composition preventing subsequent gas analysis. The new system passed exhaust gases through a particle separation aerocyclon. Particles collected on a $\mathrm{PM}_{10}$ filter paper at the bottom, whilst uninterrupted exhaust gas exit through an outlet pipe at the top directly into an exhaust gas analyser. Filter papers were dissolved using aqua regia at $150^{\circ} \mathrm{C}$ for 45 minutes in an autoclave, followed by filtering to remove any remaining filter residue. Analysis was performed using both ICP-MS, utilizing the most sensitive isotopes, and GFAAS. Detection limits $(3 \sigma)$ were calculated to be: Pd $0.03 \mu \mathrm{g} \mathrm{L}{ }^{-1}$, Pt $0.01 \mu \mathrm{g} \mathrm{L}^{-1}$, and Rh $0.002 \mu \mathrm{g} \mathrm{L}^{-1}$ for ICPMS, whilst for GFAAS they were: Pd $0.6 \mu \mathrm{g} \mathrm{L}^{-1}$, Pt $0.4 \mu \mathrm{g} \mathrm{L}^{-1}$, Rh $0.2 \mu \mathrm{g} \mathrm{L} \mathrm{L}^{-1}$. Comparison of the two techniques for the analysis of samples collected from 31 vehicles showed good agreement and suggested no systematic measurement error. Accuracy of the methodology was validated by introducing a certified reference material, BCR-CRM 723, into the system. 
Modern trends in analytical chemistry are towards the simplification and miniaturisation of sample preparation procedures. Unconventional extraction techniques have emerged including liquid-liquid extractions (LLE), cloud point extractions (CPE) and dispersive liquid-liquid micro-extractions (DLLME), that are of low cost, require relatively small sample volumes and have high enrichment factors. Two papers reported the use of DLLME as a method for the determination of trace Pd. The first used a $2,2^{\prime}$-furyldioxime chelating agent, ${ }^{328}$ while chloroform and methanol were used as extraction and dispersive solvents respectively. Analysis was performed using FAAS calibrated by spiked water samples extracted under the optimized DLLME procedure. The LOD was $0.04 \mu \mathrm{g} \mathrm{L}^{-1}(3 \sigma)$, and the relative standard deviation was $1.1 \%$ at the $4 \mu \mathrm{g} \mathrm{L} \mathrm{L}^{-1}$ level. The second paper, by Eskandari ${ }^{329}$ used 1-(2-pyridylazo)-2-naphthol dissolved in ethanol to produce a hydrophobic palladium complex that was extracted in 1-hexa-3-methylimidazolium hexafluorophosphate. For analysis, $10 \mu \mathrm{L}$ of the ionic liquid phase was mixed with $15 \mu \mathrm{L}$ ethanol, to reduce the viscosity, and injected into a GFAAS furnace tube. The LOD of the optimized method was $0.003 \mu \mathrm{g} \mathrm{L}^{-1}(3 \sigma)$, and the relative standard deviation was $1.3-4.8 \%$ over the 0.05 to $40.0 \mu \mathrm{g} \mathrm{L}^{-1}$ range. Both papers discussed optimization of reagents, extraction procedures and interfering ions, and the protocols were successfully applied to the determination of trace Pd in catalytic convertor samples after grinding and dissolution of the material in mineral acid.

3.4.3. Miscellaneous. In situ X-ray absorption spectroscopy (XAS) is a powerful tool for studying elemental-resolved structure and chemical state information of materials under simulated reaction conditions. Li et al. ${ }^{330}$ utilized this technique to study the synergistic effects of the $\mathrm{PtRh}-\mathrm{SNO}_{2}$ interaction of ternary $\mathrm{Pt}-\mathrm{Rh}-\mathrm{SnO}_{2} / \mathrm{C}$ catalysts for ethanol electro-oxidation. Samples were prepared as working electrodes on Nafion membranes and sealed in a Plexiglas cell equipped with a conventional three electrode system. The XAS spectra were collected at varying potentials under steady state conditions. Fitting of XANES and EXAFS for the Pt L3-edge showed that the Pt was initially metallic, however, oxidation begins when the potential increases above $0.41 \mathrm{~V}$. $\mathrm{Rh}$, on the other hand, readily oxidized and fitting revealed that $\mathrm{Rh}$ changed its oxidation state from $\mathrm{Rh}^{0}$ to $\mathrm{Rh}^{3+}$, without passing through reaction intermediates with lower oxidation states. It was also observed that both the Pt and $\mathrm{Rh}$ white lines (strong absorbance lines) for the catalyst were less than those measured for the respective pure foils. Since this region is characteristic of electron transitions between orbitals, it was postulated that strong interactions between the alloyed $\mathrm{Pt}$ and $\mathrm{Rh}$ resulted in electron transfer between the two.

\section{Glossary of terms}

3D Three dimensional

AAS Atomic absorption spectrometry

AFFF Asymmetric field flow fractionation

A4F Asymmetric flow field flow fractionation
AFS

AFM

AMS

APT

BCR

CCD

CRM

$\mathrm{CV}$

DLS

DMF

EDAX

EDXRD

EDXRF

ERDA

ESI-MS

ETAAS

ETV

EXAFS

FAAS

FFF

FI

FTIR

FWHM

GC

GD-MS

GD-OES

GI-SAXS

GI-XRD

GI-XRF

HG

HPLC

IAEA

ICP

ICP-MS

ICP-QMS

ICP-TOF

MS

ID

IL-

DLLME

IRMS

ISO

LA

LASIL

LEIS

LIBS

LIF

LIPS

LOD

LOQ

MALDI-

TOF

MALLS

MC

MS

MWCNT

Nd:YAG

NMR
Atomic fluorescence spectrometry

Atomic force microscopy

Accelerator mass spectrometry

Atom probe tomography

Community Bureau of Reference

Charge coupled device

Certified reference material

Cold vapour

Dynamic light scattering

$\mathrm{N}, \mathrm{N}$-dimethylformamide

Energy dispersive X-ray analysis

Energy dispersive X-ray diffraction

Energy dispersive X-ray fluorescence

Elastic recoil detection analysis

Electrospray ionization mass spectrometry

Electrothermal atomic absorption spectrometry

Electrothermal vaporization

Extended X-ray absorption fine structure

Flame atomic absorption spectrometry

Field flow fractionation

Flow injection

Fourier transform infrared

Full width at half maximum

Gas chromatography

Glow discharge mass spectrometry

Glow discharge optical emission spectrometry

Grazing incidence small angle X-ray scattering

Grazing incidence X-ray diffraction

Grazing incidence X-ray fluorescence

Hydride generation

High performance liquid chromatography

International Atomic Energy Agency

Inductively coupled plasma

Inductively coupled plasma mass spectrometry

Inductively coupled plasma quadrupole mass

spectrometry

Inductively coupled plasma-Time-of-flight mass

spectrometry

Isotope dilution

Ionic liquid-dispersive liquid liquid

microextraction

Isotope ratio mass spectrometry

International organization for standardization

Laser ablation

Laser ablation of sample in liquid

Low energy ion scattering.

Laser induced breakdown spectrometry

Laser induced fluorescence

Laser induced plasma spectroscopy

Limit of detection

Limit of quantification

Matrix assisted laser desorption ionization time-of-

flight

Multi-angle laser light scattering

Multicollector

Mass spectrometry

Multi-walled carbon nanotube

Neodymium doped:yttrium aluminium garnet

Nuclear magnetic resonance 
NRA Nuclear reaction analysis

OES Optical emission spectrometry

NIST National institute of standards and technology

PCA Principal component analysis

PGM Platinum group metals

PIGE Particle-induced gamma ray emission

PIXE Particle-induced X-ray emission

PL-PSDA Polymer Labs particle size distribution analysis

PLS Partial least squares

PLS-DA Partial least squares discriminant analysis

ppb Part per billion

ppm Part per million

RBS Rutherford backscattering spectrometry

RDA Regularized discriminant analysis

REE Rare earth element

rf Radiofrequency

RIMS Resonance ionization mass spectrometry

RSD Relative standard deviation

SEM Scanning electron microscopy

SF Sector field

SIMCA Soft independent modelling of class analogy

SIMS Secondary ion mass spectrometry

SPEM Scanning photoemission microscopy

SR Synchrotron radiation

SRM Standard reference material

SRS Synchrotron radiation source

STXM Scanning transmission X-ray microscopy

SWCNT Single-walled carbon nanotube

TEM Transmission electron microscopy

TGA Thermogravimetric analysis

TIMS Thermal ionization mass spectrometry

TPR Temperature programmed reduction

TXRF Total reflection X-ray fluorescence

UV-VIS Ultraviolet-visible

VOC Volatile organic carbon

WDXRF Wavelength dispersive X-ray fluorescence

XAFS X-ray absorption fine structure spectrometry

XANES X-ray absorption near-edge structure

XAS X-ray absorption spectroscopy

XPS X-ray photoelectron spectroscopy

XRD X-ray diffraction

XRF X-ray fluorescence

XRR X-ray reflectometry

\section{References}

1 S. Carter, A. S. Fisher, M. W. Hinds and S. Lancaster, J. Anal. At. Spectrom., 2012, 27(12), 2003-2053.

2 M. West, A. T. Ellis, P. J. Potts, C. Streli, C. Vanhoof, D. Wegrzynek and P. Wobrauschek, J. Anal. At. Spectrom., 2012, 27(10), 1603-1644.

3 E. H. Evans, C. D. Palmer and C. M. M. Smith, J. Anal. At. Spectrom., 2012, 27(6), 909-927.

4 R. Clough, L. R. Drennan-Harris, C. F. Harrington, S. J. Hill and J. F. Tyson, J. Anal. At. Spectrom., 2012, 27(8), 1185-1224.

5 A. Taylor, M. P. Day, J. Marshall, M. Patriarca and M. White, J. Anal. At. Spectrom., 2012, 27(4), 537-576.
6 S. Drensler, C. C. Mardare, S. Milenkovic and A. W. Hassel, Phys. Status Solidi A, 2012, 209(5), 854-858.

7 S. G. Gopalakrishnan, P. Huczkowski, J. Pernpeintner, T. Fend, H. Hattendorf, R. Iskandar, J. Mayer, L. Singheiser and W. J. Quadakkers, Mater. High Temp., 2012, 29(3), 249-256.

8 D. J. Mun, E. J. Shin, K. C. Cho, J. S. Lee and Y. M. Koo, Metall. Mater. Trans. A, 2012, 43A(5), 1639-1648.

9 S. Kim, Y. Kang and C. Lee, Mater. Sci. Eng., A, 2013, 559, 178-186.

10 K. J. Choi, S. H. Shin, J. J. Kim, J. A. Jung and J. H. Kim, Nucl. Eng. Technol., 2012, 44(5), 491-500.

11 S. Kaciulis, A. Mezzi, M. Amati, R. Montanari, G. Angella and M. Maldini, Surf. Interface Anal., 2012, 44(8), 982-985.

12 M. Y. Rafique, L. Q. Pan, M. Z. Iqbal, Q. U. A. Javed, H. M. Qiu, d. Rafi ud, M. H. Farooq and Z. G. Guo, J. Alloys Compd., 2013, 550, 423-430.

13 H. Itani, J. Duchoslav, M. Arndt, T. Steck, J. Gerdenitsch, J. Faderl, K. Preis, W. Winkler and D. Stifter, Anal. Bioanal. Chem., 2012, 403(3), 663-673.

14 T. Miller, J. M. Lin, L. Pirolli, L. Coquilleau, R. Luharuka and A. V. Teplyakov, Thin Solid Films, 2012, 522, 193-198.

15 B. Novotnik, T. Zuliani, J. Scancar and R. Milacic, J. Anal. At. Spectrom., 2012, 27(9), 1484-1493.

16 A. Michelin, E. Drouet, E. Foy, J. J. Dynes, D. Neff and P. Dillmann, J. Anal. At. Spectrom., 2013, 28(1), 59-66.

17 G. S. Groenewold, R. Avci, R. V. Fox, M. Deliorman, Z. Y. Suo and L. Kellerman, Ind. Eng. Chem. Res., 2013, 52(4), 13961404.

18 T. H. Kim, D. H. Lee, D. Kim, C. Jang and J. I. Yun, J. Anal. At. Spectrom., 2012, 27(9), 1525-1531.

19 I. Lopez-Quintas, M. P. Mateo, V. Pinon, A. Yanez and G. Nicolas, Spectrochim. Acta, Part B, 2012, 74-75, 109-114.

20 J. Gurell, A. Bengtson, M. Falkenstrom and B. A. M. Hansson, Spectrochim. Acta, Part B, 2012, 74-75, 46-50.

21 F. B. Gonzaga and C. Pasquini, Spectrochim. Acta, Part B, 2012, 69, 20-24.

22 H. Lefaix-Jeuland, S. Moll, F. Legendre and F. Jomard, Nucl. Instrum. Methods Phys. Res., Sect. B, 2013, 295, 69-71.

23 L. H. Wang, X. M. Lu, X. J. Wei, Z. Jiang, S. Q. Gu, Q. Gao and Y. Y. Huang, J. Anal. At. Spectrom., 2012, 27(10), 16671673.

24 Z. C. Meng and N. Zhang, Russ. J. Non-Ferrous Met., 2012, 53(4), 289-291.

25 S. Brennan, A. P. Warren, K. R. Coffey, N. Kulkarni, P. Todd, M. Kilmov and Y. Sohn, J. Phase Equilib. Diffus., 2012, 33(2), 121-125.

26 T. N. Vu, P. Volovitch and K. Ogle, Corros. Sci., 2013, 67, 4249.

27 L. J. Song and J. Mazumder, IEEE Sens. J., 2012, 12(5), 958964.

28 W. Ensinger, S. Flege and K. Baba, Nucl. Instrum. Methods Phys. Res., 2012, 272, 441-445.

29 S. L. Yang, C. R. Yu, G. W. Yang, G. Gao and L. Q. Wu, Rare Met. Mater. Eng., 2012, 41(3), 539-541.

30 Y. Kamo, M. Ueda, K. Kawamura and T. Maruyama, ISIJ Int., 2012, 52(11), 2083-2092. 
31 I. S. Molchan, S. Marimuthu, A. Mhich, Z. Liu, T. Hashimoto, G. E. Thompson, D. Whitehead, Z. B. B. Wang, P. Mativenga, L. Li, C. Grafton-Reed, I. H. Leaver, S. Cheetham and S. Dilworth, J. Anal. At. Spectrom., 2013, 28(1), 150-155.

32 C. Yong, Rare Met. Mater. Eng., 2012, 41(10), 1871-1874.

33 A. Sengupta, B. Rajeswari, R. M. Kadam and S. V. Godbole, At. Spectrosc., 2012, 33(2), 48-52.

34 P. J. Purohit, N. Goyal and S. V. Godbole, At. Spectrosc., 2012, 33(3), 92-99.

35 X. Y. Wang and H. Ren, Rare Met. Mater. Eng., 2011, 40, 18-20. 36 B. G. Oztoprak, E. Akman, M. M. Hanon, M. Gunes, S. Gumus, E. Kacar, O. Gundogdu, M. Zeren and A. Demir, Opt. Laser Technol., 2013, 45, 748-755.

37 R. J. Holmberg, S. Bolduc, D. Beauchemin, G. Jerkiewicz, H. Schulz, U. Kohlhaas and H. Strzelecki, ACS Appl. Mater. Interfaces, 2012, 4(12), 6487-6498.

38 E. Cadirli, U. Boyuk, H. Kaya and N. Marasli, Met. Mater. Int., 2012, 18(2), 349-354.

39 V. Jayan, Mater. High Temp., 2012, 29(1), 54-58.

40 G. Sutter, J. J. Arnoux, G. List, P. Bourson, S. Margueron and H. Chaynes, Tribol. Int., 2013, 57, 86-91.

41 Z. Petrova, J. Jehlicka, T. Capoun, R. Hanus, T. Trojek and V. Golias, J. Raman Spectrosc., 2012, 43(9), 1275-1280.

42 P. Colomban, A. Tournie, M. Maucuer and P. Meynard, J. Raman Spectrosc., 2012, 43(6), 799-808.

43 S. B. Li, J. Ni, X. N. Liu, H. B. Lu, S. H. Yin, M. D. Rong, Z. H. Guo and L. Zhou, Mater. Trans., 2012, 53(5), 913-919.

44 E. Chikarakara, S. Naher and D. Brabazon, Surf. Coat. Technol., 2012, 206(14), 3223-3229.

45 S. Taguchi and F. Kirino, J. Jpn. Inst. Met., 2012, 76(4), 265271.

46 L. Pardini, A. El Hassan, M. Ferretti, A. Foresta, S. Legnaioli, G. Lorenzetti, E. Nebbia, F. Catalli, M. A. Harith, D. D. Pace, F. A. Garcia, M. Scuotto and V. Palleschi, Spectrochim. Acta, Part B, 2012, 74-75, 156-161.

47 M. Rodrigues, M. Schreiner, M. Melcher, M. Guerra, J. Salomon, M. Radtke, M. Alram and N. Schindel, X-Ray Spectrom., 2012, 41(6), 416-424.

48 V. K. Gouda, G. I. Youssef and N. A. A. Ghany, Surf. Interface Anal., 2012, 44(10), 1338-1345.

49 G. Galbacs, N. Jedlinszki and A. Metzinger, Microchem. J., 2013, 107, 17-24.

50 S. Legnaioli, G. Lorenzetti, L. Pardini, V. Palleschi, D. M. D. Pace, F. A. Garcia, R. Grassi, F. Sorrentino, G. Carelli, M. Francesconi, F. Francesconi and R. Borgogni, Spectrochim. Acta, Part B, 2012, 71-72, 123-126.

51 H. Suyanto, Z. S. Lie, H. Niki, K. Kagawa, K. Fukumoto, H. Rinda, S. N. Abdulmadjid, A. M. Marpaung, M. Pardede, M. M. Suliyanti, A. N. Hidayah, E. Jobiliong, T. J. Lie, M. O. Tjia and K. H. Kurniawan, Anal. Chem., 2012, 84(5), 2224-2231.

52 R. Glaus, J. Koch and D. Gunther, Anal. Chem., 2012, 84(12), 5358-5364.

53 D. Walaszek, M. Senn, M. Faller, L. Philippe, B. Wagner, E. Bulska and A. Ulrich, Spectrochim. Acta, Part B, 2013, 79-80, 17-30.
54 L. Musilek, T. Cechak and T. Trojek, Appl. Radiat. Isot., 2012, 70(7), 1193-1202.

55 M. Guerra, M. Manso, S. Longelin, S. Pessanha and M. L. Carvalho, J. Instrum., 2012, 7, 10.

56 M. B. Dessuy, R. M. de Jesus, G. C. Brandao, S. L. C. Ferreira, M. G. R. Vale and B. Welz, Food Addit. Contam., Part A, 2013, 30(1), 202-207.

57 S. H. Zhang, X. L. Yu, F. Li, G. J. Kang, L. H. Chen and X. Y. Zhang, Opt. Lasers Eng., 2012, 50(6), 877-882.

58 M. Buschbeck, F. Buchler, T. Halfmann and S. Arndt, Spectrochim. Acta, Part B, 2012, 74-75, 103-108.

59 F. A. S. Cunha, R. A. Sousa, D. P. Harding, S. Cadore, L. F. Almeida and M. C. U. Araujo, Anal. Chim. Acta, 2012, 727, 34-40.

60 E. M. Becker, M. B. Dessuy, W. Boschetti, M. G. R. Vale, S. L. C. Ferreira and B. Welz, Spectrochim. Acta, Part B, 2012, 71-72, 102-106.

61 R. Sanchez, J. L. Todoli, C. P. Lienemann and J. M. Mermet, J. Anal. At. Spectrom., 2012, 27(6), 937-945.

62 N. Vorapalawut, M. M. Labrador, P. Pohl, M. Caetano, J. Chirinos, C. Arnaudguilhem, B. Bouyssiere, J. Shiowatana and R. Lobinski, Talanta, 2012, 97, 574578.

63 M. A. Lopez-Anton, M. Diaz-Somoano, R. Ochoa-Gonzalez and M. R. Martinez-Tarazona, Int. J. Coal Geol., 2012, 94, 44-53.

64 L. Zhang, Z. Y. Hu, W. B. Yin, D. Huang, W. G. Ma, L. Dong, H. P. Wu, Z. X. Li, L. T. Xiao and S. T. Jia, Front. Phys., 2012, $7(6), 690-700$.

65 T. B. Yuan, Z. Wang, L. Z. Li, Z. Y. Hou, Z. Li and W. D. Ni, Appl. Opt., 2012, 51(7), B22-B29.

66 Z. Wang, T. B. Yuan, S. L. Lui, Z. Y. Hou, X. W. Li, Z. Li and W. D. Ni, Front. Phys., 2012, 7(6), 708-713.

67 M. R. Dong, X. L. Mao, J. J. Gonzalez, J. D. Lu and R. E. Russo, J. Anal. At. Spectrom., 2012, 27(12), 2066-2075.

68 S. Q. Guo, J. L. Yang and Z. Y. Liu, Energy Fuels, 2012, 26(6), 3388-3392.

69 F. Low and L. Zhang, Talanta, 2012, 101, 346-352.

70 M. Mujuru, S. Moyo, R. I. McCrindle and N. MokgalakaMatlala, Spectrochim. Acta, Part B, 2012, 76, 147-151.

71 J. Lusilao-Makiese, E. Tessier, D. Amouroux, H. Tutu, L. Chimuka and E. M. Cukrowska, Toxicol. Environ. Chem., 2012, 94(9), 1688-1706.

72 P. A. Mello, J. S. F. Pereira, M. F. Mesko, J. S. Barin and E. M. M. Flores, Anal. Chim. Acta, 2012, 746, 15-36.

73 G. S. Ortega, C. Pecheyran, G. Hudin, E. Marosits and O. F. X. Donard, Microchem. J., 2013, 106, 250-254.

74 F. V. M. Pontes, M. C. Carneiro, D. S. Vaitsman, M. I. C. Monteiro, A. A. Neto, M. L. B. Tristao and M. D. Guerrante, Fuel Process. Technol., 2013, 106, 122-126.

75 A. Cinosi, N. Andriollo, F. Tibaldi and D. Monticelli, Talanta, 2012, 101, 148-150.

76 F. I. de Albuquerque, C. B. Duyck, T. C. O. Fonseca and T. D. Saint'Pierre, Spectrochim. Acta, Part B, 2012, 71-72, 112-116.

77 G. Sanabria-Ortega, C. Pecheyran, S. Berail and O. F. X. Donard, Anal. Chem., 2012, 84(18), 7874-7880. 
78 G. S. Ortega, C. Pecheyran, S. Berail and O. F. X. Donard, J. Anal. At. Spectrom., 2012, 27(9), 1447-1456.

79 H. M. Pessoa, R. A. Hauser-Davis, R. C. de Campos, E. V. R. de Castro, M. Carneiro and G. P. Brandao, J. Anal. At. Spectrom., 2012, 27(9), 1568-1573.

80 S. Carballo-Paradelo, R. M. Soto-Ferreiro, A. CarlosenaZubieta, J. Teran-Baamonde, J. M. Andrade-Garda and D. Prada-Rodriguez, J. Anal. At. Spectrom., 2012, 27(10), 1694-1700.

81 A. de Jesus, A. V. Zmozinski, I. C. F. Damin, M. M. Silva and M. G. R. Vale, Spectrochim. Acta, Part B, 2012, 71-72, 86-91.

82 F. V. M. Pontes, M. C. Carneiro, D. S. Vaitsman, M. I. C. Monteiro, A. A. Neto, E. D. F. de Souza and M. L. B. Tristao, J. Anal. At. Spectrom., 2012, 27(10), 17941798.

83 M. Martinez, C. Arnaudguilhem, R. Lobinski, B. Bouyssiere, M. Caetano and J. Chirinos, J. Anal. At. Spectrom., 2012, 27(6), 1007-1011.

84 Y. B. Zhu, T. Narukawa, M. Numata, Y. Kitamaki, M. Matsuo, A. Hioki, K. Kato and K. Chiba, Fuel, 2013, 103, 736-741.

85 R. S. Amais, G. L. Donati, D. Schiavo and J. A. Nobrega, Microchem. J., 2013, 106, 318-322.

86 L. F. S. Caldas, C. E. R. de Paula, D. M. Brum and R. J. Cassella, Fuel, 2013, 105, 503-511.

87 J. R. de Souza, C. B. Duyck, T. C. O. Fonseca and T. D. Saint'Pierre, J. Anal. At. Spectrom., 2012, 27(8), 12801286.

88 P. R. Aranda, J. A. Gasquez, R. A. Olsina, L. D. Martinez and R. A. Gil, Talanta, 2012, 101, 353-356.

89 G. L. Donati, R. S. Amais and J. A. Nobrega, J. Anal. At. Spectrom., 2012, 27(8), 1274-1279.

90 D. R. Neves, R. S. Amais, J. A. Nobrega and J. A. G. Neto, Anal. Lett., 2012, 45(9), 1111-1121.

91 L. S. G. Teixeira, E. S. Santos and L. S. Nunes, Anal. Chim. Acta, 2012, 722, 29-33.

92 J. Cortez and C. Pasquini, Anal. Chem., 2013, 85(3), 15471554.

93 M. A. Aguirre, N. Kovachev, M. Hidalgo and A. Canals, J. Anal. At. Spectrom., 2012, 27(12), 2102-2110.

94 C. S. Da Silva, E. Q. Oreste, A. M. Nunes, M. A. Vieira and A. S. Ribeiro, J. Anal. At. Spectrom., 2012, 27(4), 689-694.

95 M. L. Franquelo, A. Duran, J. Castaing, D. Arquillo and J. L. Perez-Rodriguez, Talanta, 2012, 89, 462-469.

96 G. Sciutto, P. Oliveri, S. Prati, M. Quaranta, S. Bersani and R. Mazzeo, Anal. Chim. Acta, 2012, 752, 30-38.

97 M. Alfeld, W. De Nolf, S. Cagno, K. Appel, D. P. Siddons, A. Kuczewski, K. Janssens, J. Dik, K. Trentelman, M. Walton and A. Sartorius, J. Anal. At. Spectrom., 2013, 28(1), 40-51.

98 I. Cianchetta, I. Colantoni, F. Talarico, F. d'Acapito, A. Trapananti, C. Maurizio, S. Fantacci and I. Davoli, J. Anal. At. Spectrom., 2012, 27(11), 1941-1948.

99 A. Pitarch, A. Alvarez-Perez, K. Castro, J. M. Madariaga and I. Queralt, J. Raman Spectrosc., 2012, 43(3), 411-418.

100 A. Le Gac, R. Estrompa, J. C. Frade, S. Pessanha, T. I. Madeira, A. Cardoso, L. Piorro, L. Dias, J. Mirao,
A. Candeias and M. L. Carvalho, J. Anal. At. Spectrom., 2012, 27(4), 626-643.

101 F. Caruso, S. Saverwyns, M. Van Bos, D. F. C. Martino, A. E. Ceulemans, J. de Valck and E. Caponetti, Appl. Phys. A: Mater. Sci. Process., 2012, 107(1), 197-202.

102 D. M. Goltz, Anal. Lett., 2012, 45(4), 314-329.

103 K. L. Rasmussen, A. L. Tenorio, I. Bonaduce, M. P. Colombini, L. Birolo, E. Galano, A. Amoresano, G. Doudna, A. D. Bond, V. Palleschi, G. Lorenzetti, S. Legnaioli, J. van der Plicht and J. Gunneweg, J. Archaeol. Sci., 2012, 39(9), 2956-2968.

104 K. Nesmerak and I. Nemcova, Anal. Lett., 2012, 45(4), 330344.

105 M. Alfeld, D. P. Siddons, K. Janssens, J. Dik, A. Woll, R. Kirkham and E. van de Wetering, Appl. Phys. A: Mater. Sci. Process., 2013, 111(1), 157-164.

106 D. L. Howard, M. D. de Jonge, D. Lau, D. Hay, M. VarcoeCocks, C. G. Ryan, R. Kirkham, G. Moorhead, D. Paterson and D. Thurrowgood, Anal. Chem., 2012, 84(7), 32783286.

107 J. L. Mass, R. Opila, B. Buckley, M. Cotte, J. Church and A. Mehta, Appl. Phys. A: Mater. Sci. Process., 2013, 111(1), 59-68.

108 N. Salvado, S. Buti, M. Cotte, G. Cinque and T. Pradell, Appl. Phys. A: Mater. Sci. Process., 2013, 111(1), 47-57.

109 F. Casadio and V. Rose, Appl. Phys. A: Mater. Sci. Process., 2013, 111(1), 1-8.

110 I. Reiche, K. Muller, M. Eveno, E. Itie and M. Menu, J. Anal. At. Spectrom., 2012, 27(10), 1715-1724.

111 L. Bertrand, M. Cotte, M. Stampanoni, M. Thoury, F. Marone and S. Schoder, Phys. Rep., 2012, 519(2), 51-96.

112 M. F. Gazulla, S. Vicente, M. Orduna and J. Ventura, X-Ray Spectrom., 2012, 41(3), 176-185.

113 F. D. L. Borba, J. Cortez, V. K. Asfora, C. Pasquini, M. F. Pimentel, A. M. Pessis and H. J. Khoury, J. Braz. Chem. Soc., 2012, 23(5), 958-965.

114 Q. Q. Wang, K. Liu, H. Zhao, C. H. Ge and Z. W. Huang, Front. Phys., 2012, 7(6), 701-707.

115 F. C. De Lucia and J. L. Gottfried, Appl. Opt., 2012, 51(7), B83-B92.

116 J. L. Gottfried, Appl. Opt., 2013, 52(4), B10-B19.

117 Q. Q. Wang, K. Liu and H. Zhao, Chin. Phys. Lett., 2012, 29(4), 3.

118 J. Moros, J. Serrano, C. Sanchez, J. Macias and J. J. Laserna, J. Anal. At. Spectrom., 2012, 27(12), 2111-2122.

119 A. Fernandez-Bravo, P. Lucena and J. J. Laserna, Appl. Spectrosc., 2012, 66(10), 1197-1203.

120 J. L. Gottfried, Appl. Opt., 2012, 51(7), B13-B21.

121 N. C. Dingari, I. Barman, A. K. Myakalwar, S. P. Tewari and M. K. Gundawar, Anal. Chem., 2012, 84(6), 2686-2694.

122 V. U. Contreras, M. A. Meneses-Nava, N. Ornelas-Soto, O. Barbosa-Garcia, P. L. Lopez-de-Alba, J. L. Maldonado, G. Ramos-Ortiz, F. J. Acevedo-Aguilar and L. LopezMartinez, Appl. Spectrosc., 2012, 66(11), 1294-1301.

123 A. Albert, C. Brauckmann, F. Blaske, M. Sperling, C. Engelhard and U. Karst, J. Anal. At. Spectrom., 2012, 27(6), 975-981. 
124 C. Losada, J. J. Alberti, J. Saurina and S. Sentellas, Anal. Bioanal. Chem., 2012, 404(2), 539-551.

125 F. Seby, C. Gleyzes, O. Grosso, B. Plau and O. F. X. Donard, Anal. Bioanal. Chem., 2012, 404(10), 2939-2948.

126 F. van Heuveln, H. Meijering and J. Wieling, Bioanalysis, 2012, 4(15), 1933-1965.

127 B. Meermann and M. Sperling, Anal. Bioanal. Chem., 2012, 403(6), 1501-1522.

128 N. Ozbek and S. Akman, Talanta, 2012, 94, 246-250.

129 T. Limburg and J. W. Einax, Microchem. J., 2013, 107, 31-36.

130 B. J. Shaw, D. J. Semin, M. E. Rider and M. R. Beebe, J. Pharm. Biomed. Anal., 2012, 63, 151-159.

131 A. Meyer, S. Grotefend, A. Gross, H. Watzig and I. Ott, J. Pharm. Biomed. Anal., 2012, 70, 713-717.

132 H. Y. He, Z. L. Zhu, H. T. Zheng, Q. Xiao, L. L. Jin and S. H. Hu, Microchem. J., 2012, 104, 7-11.

133 Y. Gao, X. H. Peng, Z. M. Shi, R. X. Zhang, X. F. Xia, F. R. Yue and R. Liu, At. Spectrosc., 2012, 33(3), 73-77.

134 L. Hao, H. Y. Cheng, J. H. Liu and X. F. Yin, Chem. J. Chin. Univ., 2012, 33(9), 1957-1963.

135 A. Asfaw, G. Wibetoe and D. Beauchemin, J. Anal. At. Spectrom., 2012, 27(11), 1928-1934.

136 S. Gunduz and S. Akman, Regul. Toxicol. Pharmacol., 2013, 65(1), 34-37.

137 A. L. H. Muller, P. A. Mello, M. F. Mesko, F. A. Duarte, V. L. Dressler, E. I. Muller and E. M. M. Flores, J. Anal. At. Spectrom., 2012, 27(11), 1889-1894.

138 K. Van Hoecke, C. Catry and F. Vanhaecke, J. Anal. At. Spectrom., 2012, 27(11), 1909-1919.

139 E. Mladenova, I. Karadjova and D. L. Tsalev, J. Sep. Sci., 2012, 35(10-11), 1249-1265.

140 E. G. da Silva, F. Augusto and M. A. Z. Arruda, Analyst, 2012, 137(16), 3841-3846.

141 A. A. Alqadami, M. A. Abdalla, Z. A. Alothman and K. Omer, Int. J. Environ. Res. Public Health, 2013, 10(1), 361-374.

142 C. E. R. de Paula, L. F. S. Caldas, D. M. Brum and R. J. Cassella, J. Pharm. Biomed. Anal., 2013, 74, 284-290.

143 P. H. van Wyk, W. J. Gerber and K. R. Koch, J. Anal. At. Spectrom., 2012, 27(4), 577-580.

144 F. Varenne, M. Bourdillon, M. Meyer, Y. Lin, M. Brellier, R. Baati, L. J. Charbonniere, A. Wagner, E. Doris, F. Taran and A. Hagege, J. Chromatogr., A, 2012, 1229, 280-287.

145 L. Telgmann, H. Faber, S. Jahn, D. Melles, H. Simon, M. Sperling and U. Karst, J. Chromatogr., A, 2012, 1240, 147-155.

146 H. Y. Cheng, J. H. Liu, X. F. Yin, H. Shen and Z. G. Xu, Analyst, 2012, 137(13), 3111-3118.

147 S. Latzel, D. Neimke, R. Schumacher, M. Barth and L. Niewohner, Forensic Sci. Int., 2012, 223(1-3), 273-278.

148 Z. Abrego, A. Ugarte, N. Unceta, A. Fernandez-Isla, M. A. Goicolea and R. J. Barrio, Anal. Chem., 2012, 84(5), 2402-2409.

149 A. Taborelli, D. Gibelli, A. Rizzi, S. Andreola, A. Brandone and C. Cattaneo, J. Forensic Sci., 2012, 57(5), 1281-1284.

150 C. N. Rinke, M. R. Williams, C. Brown, M. Baudelet, M. Richardson and M. E. Sigman, Anal. Chim. Acta, 2012, 753, 19-26.
151 K. E. Eseller, F. Y. Yueh, J. P. Singh and N. Melikechi, Appl. Opt., 2012, 51(7), B171-B175.

152 M. M. Tripathi, K. E. Eseller, F. Y. Yueh and J. P. Singh, Sens. Actuators, B, 2012, 171, 416-422.

153 H. Heilbrunner, N. Huber, H. Wolfmeir, E. Arenholz, J. D. Pedarnig and J. Heitz, Spectrochim. Acta, Part B, 2012, 74-75, 51-56.

154 M. M. Suliyanti, A. N. Hidayah, M. Pardede, E. Jobiliong, S. N. Abdulmadjid, N. Idris, M. Ramli, T. J. Lie, R. Hedwig, M. O. Tjia, K. H. Kurniawan, Z. S. Lie, H. Niki and K. Kagawa, Spectrochim. Acta, Part B, 2012, 69, 56-60.

155 S. Darwiche, R. Benrabbah, M. Benmansour and D. Morvan, Spectrochim. Acta, Part B, 2012, 74-75, 115-118.

156 M. Hornackova, Z. Grolmusova, M. Hornacek, J. Rakovsky, P. Hudec and P. Veis, Spectrochim. Acta, Part B, 2012, 74-75, 119-123.

157 J. Zhang, G. Ma, H. H. Zhu, J. H. Xi and Z. G. Ji, J. Anal. At. Spectrom., 2012, 27(11), 1903-1908.

158 W. A. Farooq, F. N. Al-Mutairi, A. E. M. Khater, A. S. AlDwayyan, M. S. AlSalhi and M. Atif, Opt. Spectrosc., 2012, 112(6), 874-880.

159 M. A. Gondal, M. A. Dastageer, A. A. Naqvi, A. A. Isab and Y. W. Maganda, Appl. Opt., 2012, 51(30), 7395-7401.

160 E. U. Sagin, H. Boke, N. Aras and S. Yalcin, Mater. Struct., 2012, 45(6), 841-849.

161 B. Novotnik, T. Zuliani, A. Martincic, J. Scancar and R. Milacic, Talanta, 2012, 99, 83-90.

162 J. Vogl and M. Rosner, Geostand. Geoanal. Res., 2012, 36(2), 161-175.

163 E. Gautier, R. Garavaglia, A. Lobo, M. Fernandez and H. Farach, J. Anal. At. Spectrom., 2012, 27(5), 881-883.

164 M. H. Huyskens, T. Iizuka and Y. Amelin, J. Anal. At. Spectrom., 2012, 27(9), 1439-1446.

165 H. J. Heinrich and H. Kipphardt, Spectrochim. Acta, Part B, 2012, 70, 68-73.

166 M. Resano, E. Mozas, C. Crespo, J. Perez, E. Garcia-Ruiz and M. A. Belarra, Spectrochim. Acta, Part B, 2012, 71-72, 24-30.

167 N. Rey-Raap and A. Gallardo, Waste Manage., 2012, 32(5), 944-948.

168 M. Soylak and I. Murat, Food Anal. Methods, 2012, 5(5), 1003-1009.

169 S. Bucker and J. Acker, Talanta, 2012, 94, 335-341.

170 R. Castell, E. D. Greaves, L. Abdala and H. Barros, Appl. Phys. B: Lasers Opt., 2012, 109(1), 47-53.

171 L. Bencs, K. Gyorgy, M. Kardos, J. Osan, B. Alfoldy, I. Varga, Z. Ajtony, N. Szoboszlai, Z. Stefanka, E. Szeles and L. Kovacs, Anal. Chim. Acta, 2012, 726, 1-8.

172 D. R. Baer, Surface and I. Analysis, 2012, 44(9), 1305-1308. 173 M. Senoner and W. E. S. Unger, J. Anal. At. Spectrom., 2012, 27(7), 1050-1068.

174 P. Krystek, Microchem. J., 2012, 105, 39-43.

175 A. Ulrich, S. Losert, N. Bendixen, A. Al-Kattan, H. Hagendorfer, B. Nowack, C. Adlhart, J. Ebert, M. Lattuada and K. Hungerbuhler, J. Anal. At. Spectrom., 2012, 27(7), 1120-1130.

176 H. Hagendorfer, R. Kaegi, M. Parlinska, B. Sinnet, C. Ludwig and A. Ulrich, Anal. Chem., 2012, 84(6), 2678-2685. 
177 K. Loeschner, J. Navratilova, S. Legros, S. Wagner, R. Grombe, J. Snell, F. von der Kammer and E. H. Larsen, J. Chromatogr., A, 2013, 1272, 116-125.

178 J. Gigault and V. A. Hackley, Anal. Chim. Acta, 2013, 763, 57-66. 179 M. E. Hoque, K. Khosravi, K. Newman and C. D. Metcalfe, J. Chromatogr., A, 2012, 1233, 109-115.

180 E. P. Gray, T. A. Bruton, C. P. Higgins, R. U. Halden, P. Westerhoff and J. F. Ranville, J. Anal. At. Spectrom., 2012, 27(9), 1532-1539.

181 V. Nischwitz and H. Goenaga-Infante, J. Anal. At. Spectrom., 2012, 27(7), 1084-1092.

182 D. M. Mitrano, A. Barber, A. Bednar, P. Westerhoff, C. P. Higgins and J. F. Ranville, J. Anal. At. Spectrom., 2012, 27(7), 1131-1142.

183 A. C. E. Chia, J. P. Boulanger and R. R. LaPierre, Nanotechnology, 2013, 24(4), 7.

184 C. K. Ling, S. V. Verkhoturov, L. J. Chen and E. A. Schweikert, Int. J. Mass Spectrom., 2013, 334, 43-48.

185 Y. Y. Zhang, G. Xiong, S. Q. Li, Z. Z. Dong, S. G. Buckley and S. D. Tse, Combust. Flame, 2013, 160(3), 725-733.

186 P. K. Diwakar, K. H. Loper, A. M. Matiaske and D. W. Hahn, J. Anal. At. Spectrom., 2012, 27(7), 1110-1119.

187 G. Hartmann and M. Schuster, Anal. Chim. Acta, 2013, 761, 27-33.

188 S. M. Majedi, H. K. Lee and B. C. Kelly, Anal. Chem., 2012, 84(15), 6546-6552.

189 J. Soto-Alvaredo, M. Montes-Bayon and J. Bettmer, Anal. Chem., 2013, 85(3), 1316-1321.

190 W. Jiang, D. B. Hibbert, G. Moran and R. Akter, J. Anal. At. Spectrom., 2012, 27(9), 1465-1473.

191 S. Elzey, D. H. Tsai, L. L. Yu, M. R. Winchester, M. E. Kelley and V. A. Hackley, Anal. Bioanal. Chem., 2013, 405(7), 22792288.

192 A. Murtazin, S. Groh and K. Niemax, Spectrochim. Acta, Part $B, 2012,67,3-16$.

193 R. B. Reed, C. P. Higgins, P. Westerhoff, S. Tadjiki and J. F. Ranville, J. Anal. At. Spectrom., 2012, 27(7), 1093-1100.

194 H. E. Pace, N. J. Rogers, C. Jarolimek, V. A. Coleman, E. P. Gray, C. P. Higgins and J. F. Ranville, Environ. Sci. Technol., 2012, 46(22), 12272-12280.

195 S. A. Pergantis, T. L. Jones-Lepp and E. M. Heithmar, Anal. Chem., 2012, 84(15), 6454-6462.

196 J. W. Olesik and P. J. Gray, J. Anal. At. Spectrom., 2012, 27(7), 1143-1155.

197 B. Franze, I. Strenge and C. Engelhard, J. Anal. At. Spectrom., 2012, 27(7), 1074-1083.

198 F. Aureli, M. D'Amato, B. De Berardis, A. Raggi, A. C. Turco and F. Cubadda, J. Anal. At. Spectrom., 2012, 27(9), 15401548.

199 J. Tuoriniemi, G. Cornelis and M. Hassellov, Anal. Chem., 2012, 84(9), 3965-3972.

200 S. Merchel, W. Bremser, S. Akhmadaliev, M. Arnold, G. Aumaitre, D. L. Bourles, R. Braucher, M. Caffee, M. Christl, L. K. Fifield, R. C. Finkel, S. Freeman, A. RuizGomez, P. W. Kubik, M. Martschini, D. H. Rood, S. G. Tims, A. Wallner, K. M. Wilcken and S. Xu, Nucl. Instrum. Methods Phys. Res., Sect. B, 2012, 289, 68-73.
201 Z. Varga, A. Nichol, M. Wallenius and K. Mayer, Anal. Chim. Acta, 2012, 718, 25-31.

202 K. Mathew, P. Mason, A. Voeks and U. Narayanan, Int. J. Mass Spectrom., 2012, 315, 8-14.

203 S. Caroli, M. Forte, C. Nuccetelli, R. Rusconi and S. Risica, Microchem. J., 2013, 107, 95-100.

204 P. Thakur and G. P. Mulholland, Appl. Radiat. Isot., 2012, 70(8), 1747-1778.

205 A. L. de Souza, M. E. B. Cotrim and M. A. F. Pires, Microchem. J., 2013, 106, 194-201.

206 F. E. Stanley, J. Anal. At. Spectrom., 2012, 27(11), 18211830.

207 R. C. Marin, J. E. S. Sarkis and M. R. L. Nascimento, J. Radioanal. Nucl. Chem., 2013, 295(1), 99-104.

208 M. Kraiem, S. Richter, N. Erdmann, H. Kuhn, M. Hedberg and Y. Aregbe, Anal. Chim. Acta, 2012, 748, 37-44.

209 T. Shinonaga, D. Donohue, H. Aigner, S. Burger, D. Klose, T. Karkela, R. Zilliacus, A. Auvinen, O. Marie and F. Pointurier, Anal. Chem., 2012, 84(6), 2638-2646.

210 I. M. Sato, L. A. T. Pereira, M. A. Scapin, M. B. Cotrim, C. S. Mucsi, J. L. Rossi and L. G. Martinez, J. Radioanal. Nucl. Chem., 2012, 294(2), 283-288.

211 S. Dhara, N. L. Misra, U. K. Thakur, D. Shah, R. M. Sawant, K. L. Ramakumar and S. K. Aggarwal, X-Ray Spectrom., 2012, 41(5), 316-320.

212 N. L. Misra, M. K. Tiwari, S. S. Kumar, S. Dhara, A. K. Singh, G. S. Lodha, S. K. Deb, P. D. Gupta and S. K. Aggarwal, X-Ray Spectrom., 2013, 42(1), 4-7.

213 A. Sengupta, V. C. Adya and S. V. Godbole, J. Radioanal. Nucl. Chem., 2012, 292(3), 1259-1264.

214 C. Krishnakumar, P. Revathi, K. K. Nandy, K. S. Kumar, T. Kumar, J. K. Gayen, B. Shreekumar and P. K. Dey, J. Radioanal. Nucl. Chem., 2013, 295(2), 1019-1022.

215 M. Z. Martin, S. Allman, D. J. Brice, R. C. Martin and N. O. Andre, Spectrochim. Acta, Part B, 2012, 74-75, 177183.

216 S. Almaviva, L. Caneve, F. Colao, R. Fantoni and G. Maddaluno, Chem. Phys., 2012, 398, 228-232.

217 P. Ko, K. C. Hartig, J. P. McNutt, R. B. D. Schur, T. W. Jacomb-Hood and I. Jovanovic, Rev. Sci. Instrum., 2013, 84(1), 6.

218 N. Trautmann and K. Wendt, Radiochim. Acta, 2012, 100(89), 675-685.

219 K. Wendt, C. Geppert, C. Mattolat, G. Passler, S. Raeder, F. Schwellnus, K. Wies and N. Trautmann, Anal. Bioanal. Chem., 2012, 404(8), 2173-2176.

220 W. T. Lu, P. Collon, Y. Kashiv, M. Bowers, D. Robertson and C. Schmitt, Nucl. Instrum. Methods Phys. Res., Sect. B, 2013, 294, 392-396.

221 N. Stobener, S. Amayri, A. Gehl, U. Kaplan, K. Malecha and T. Reich, Anal. Bioanal. Chem., 2012, 404(8), 2143-2150.

222 S. Eichler, M. Vosmanska, E. Hofmanova, R. Koplik and O. Mestek, Chem. Listy, 2012, 106(3), 189-194.

223 M. Krachler, R. Alvarez-Sarandes and S. Van Winckel, J. Anal. At. Spectrom., 2013, 28(1), 114-120.

224 P. van Calsteren and L. Thomas, J. Anal. At. Spectrom., 2012, 27(6), 952-956. 
225 S. A. Ansari, S. Chaudhury, P. K. Mohapatra, S. K. Aggarwal and V. K. Manchanda, Sep. Sci. Technol., 2012, 48(2), 208214.

226 C. G. Lee, D. Suzuki, Y. Saito-Kokubu, F. Esaka, M. Magara and T. Kimura, Int. J. Mass Spectrom., 2012, 314, 57-62.

227 F. Esaka, C. G. Lee, M. Magara and T. Kimura, Anal. Chim. Acta, 2012, 721, 122-128.

228 B. Marchand, N. Moncoffre, Y. Pipon, C. Garnier, N. Bererd, C. Delafoy, M. Fraczkiewicz, A. Perrat-Mabillon, L. Raimbault, P. Sainsot and N. Toulhoat, Prog. Nucl. Energy, 2012, 57, 145-149.

229 T. Vaculovic, T. Warchilova, T. Simo, O. Matal, V. Otruba, P. Mikuska and V. Kanicky, J. Anal. At. Spectrom., 2012, 27(8), 1321-1326.

230 M. Krachler, S. Van Winckel, M. Cardinale, B. Lynch and T. Murakami, Microchem. J., 2012, 105, 9-14.

231 S. Durani, M. Krishnakumar and K. Satyanarayana, J. Radioanal. Nucl. Chem., 2012, 294(2), 215-220.

232 Y. Cai, P. C. Chu, S. K. Ho and N. H. Cheung, Front. Phys., 2012, 7(6), 670-678.

233 L. Balika, C. Focsa, S. Gurlui, S. Pellerin, N. Pellerin, D. Pagnon and M. Dudeck, Spectrochim. Acta, Part B, 2012, 74-75, 184-189.

234 M. A. Idris, T. Bak, S. Li and J. Nowotny, J. Phys. Chem. C, 2012, 116(20), 10950-10958.

235 P. S. Lysaght, J. C. Woicik, M. A. Sahiner, J. Price, C. Weiland and P. D. Kirsch, J. Appl. Phys., 2012, 112(6), 8.

236 M. E. V. Tagle, M. T. L. Marin, O. M. Gavilan, M. D. D. Rodriguez, F. C. Pinar and M. S. P. Alfonso, Talanta, 2012, 94, 50-57.

237 A. Zucchiatti and F. Agullo-Lopez, Nucl. Instrum. Methods Phys. Res., 2012, 278, 106-114.

238 F. Bardelli, G. Barone, V. Crupi, F. Longo, G. Maisano, D. Majolino, P. Mazzoleni and V. Venuti, J. Synchrotron Radiat., 2012, 19, 782-788.

239 M. O. Figueiredo, T. P. Silva and J. P. Veiga, J. Electron Spectrosc. Relat. Phenom., 2012, 185(3-4), 97-102.

240 A. Guilherme, G. Buzanich, M. Radtke, U. Reinholz, J. Coroado, J. M. F. Dos Santos and M. L. Carvalho, J. Anal. At. Spectrom., 2012, 27(6), 966-974.

241 S. Roncevic, L. P. Svedruzic, Z. S. Strukil and I. M. Caksiran, Anal. Methods, 2012, 4(8), 2506-2514.

242 P. Holakooei, F. C. Petrucci, R. Tassinari and C. Vaccaro, $X$-Ray Spectrom., 2013, 42(2), 105-115.

243 H. Gondai, Y. Shindo, M. Kawatoko and I. Nakai, Bunseki Kagaku, 2013, 62(2), 143-154.

244 E. Aquilia, G. Barbera, G. Barone, V. Crupi, F. Longo, D. Majolino, P. Mazzoleni and V. Venuti, X-Ray Spectrom., 2013, 42(1), 38-44.

245 V. Renson, A. Martinez-Cortizas, N. Mattielli, J. Coenaerts, C. Sauvage, F. De Vleeschouwer, C. Lorre, F. Vanhaecke, R. Bindler, M. Rautman, K. Nys and P. Claeys, Appl. Geochem., 2013, 28, 220-234.

246 M. D. Gajic-Kvascev, M. D. Maric-Stojanovic, R. M. JancicHeinemann, G. S. Kvascev and V. D. Andric, Chem. Cent. J., 2012, 6, 9.
247 J. M. Miao, C. L. Lv, H. Li and T. M. Chen, Archaeometry, 2012, 54, 955-973.

248 E. Aquilia, G. Barone, P. Mazzoleni and C. Ingoglia, J. Cult. Heritage, 2012, 13(4), 442-447.

249 D. Konig and V. Serneels, J. Archaeol. Sci., 2013, 40(1), 156165.

250 M. Golitko and J. E. Terrell, J. Archaeol. Sci., 2012, 39(12), 3568-3580.

251 B. A. Alex, D. L. Nichols and M. D. Glascock, Archaeometry, 2012, 54, 821-834.

252 S. Ichikawa, K. Nakayama and T. Nakamura, X-Ray Spectrom., 2012, 41(5), 288-297.

253 A. J. Finlay, J. M. McComish, C. J. Ottley, C. R. Bates and D. Selby, J. Archaeol. Sci., 2012, 39(7), 2385-2391.

254 T. Q. Zhu, W. D. Sun, H. Zhang, G. R. Kuang and L. B. Lv, Archaeometry, 2012, 54, 475-488.

255 A. Issi, Ceram. Int., 2012, 38(3), 2375-2380.

256 D. Frankel and J. M. Webb, J. Archaeol. Sci., 2012, 29(5), 1380-1387.

257 H. J. Ma, J. Zhu, J. Henderson and N. S. Li, J. Archaeol. Sci., 2012, 29(5), 1218-1226.

258 K. Blagoev, M. Grozeva, G. Malcheva and S. Neykova, Spectrochim. Acta, Part B, 2013, 79-80, 39-43.

259 I. Osticioli, J. Agresti, C. Fornacelli, I. T. Memmi and S. Siano, J. Anal. At. Spectrom., 2012, 27(5), 827-833.

260 W. D. Stoner and M. D. Glascock, J. Archaeol. Sci., 2012, 39(8), 2668-2683.

261 Y. Zhang, Y. H. Jia, J. W. Chen, Y. Liu, X. J. Shen, L. Zhao, S. M. Wang, H. Yu, P. C. Han, H. Y. Qu and S. Z. Liu, Guang Pu Xue Yu Guang Pu Fen Xi, 2012, 32(6), 1441-1446.

262 K. J. Kim, W. E. S. Unger, J. W. Kim, D. W. Moon, T. Gross, V. D. Hodoroaba, D. Schmidt, T. Wirth, W. Jordaan, M. van Staden, S. Prins, L. Zhang, T. Fujimoto, X. P. Song and H. Wang, Surf. Interface Anal., 2012, 44(2), 192-199.

263 M. Fallqvist, S. Ruppi, M. Olsson, M. Ottosson and T. M. Grehk, Surf. Coat. Technol., 2012, 207, 254-261.

264 J. Wang, X. J. Shi and J. Zhu, Appl. Surf. Sci., 2013, 265, 399404.

265 A. A. Shaltout, H. H. Afify and S. A. Ali, J. Electron Spectrosc. Relat. Phenom., 2012, 185(5-7), 140-145.

266 D. Q. Yuan, Lasers Eng., 2012, 23(3-4), 255-264.

267 S. H. Lee, H. S. Shim, C. K. Kim, J. H. Yoo, R. E. Russo and S. Jeong, Appl. Opt., 2012, 51(7), B115-B120.

268 C. K. Kim, D. S. Kim, S. H. Lee, H. S. Shim and S. Jeong, Appl. Opt., 2012, 51(7), B93-B98.

269 D. Mukherjee, R. Hyde, P. Mukherjee, H. Srikanth and S. Witanachchi, J. Appl. Phys., 2012, 111(6), 10.

270 K. P. Nayak, M. Das, F. L. Kien and K. Hakuta, Opt. Commun., 2012, 285(23), 4698-4704.

271 Y. Cui, J. F. Moore, S. Milasinovic, Y. M. Liu, R. J. Gordon and L. Hanley, Rev. Sci. Instrum., 2012, 83(9), 7.

272 B. Hattendorf, J. Pisonero, D. Gunther and N. Bordel, Anal. Chem., 2012, 84(20), 8771-8776.

273 M. P. Mateo, V. Pinon and G. Nicolas, Surf. Coat. Technol., 2012, 211, 89-92.

274 F. Gontad, A. Lorusso and A. Perrone, Thin Solid Films, 2012, 520(16), 5211-5214. 
275 J. Pisonero, R. Valledor, A. Licciardello, C. Quiros, J. I. Martin, A. Sanz-Medel and N. Bordel, Anal. Bioanal. Chem., 2012, 403(8), 2437-2448.

276 K. G. Reinsberg, C. Schumacher, A. Tempez, K. Nielsch and J. A. C. Broekaert, Spectrochim. Acta, Part B, 2012, 76, 175180.

277 V. Efimova, V. Hoffmann and J. Eckert, Spectrochim. Acta, Part B, 2012, 76, 181-189.

278 S. Mushtaq, V. Hoffmann, E. B. M. Steers and J. C. Pickering, J. Anal. At. Spectrom., 2012, 27(9), 1423-1431. 279 I. S. Molchan, G. E. Thompson, P. Skeldon, A. Licciardello, N. Tuccitto and A. Tempez, J. Anal. At. Spectrom., 2013, 28(1), 121-126.

280 C. C. Du, T. C. Wei, C. H. Chang, S. L. Lee, M. W. Liang, J. R. Huang, C. H. Wu, A. Shirakura, R. Morisawa and T. Suzuki, Thin Solid Films, 2012, 520(11), 3999-4002.

281 M. West, Glass Technol.: Eur. J. Glass Sci. Technol., Part A, 2012, 53(5), 198-201.

282 A. V. Chadwick, A. Berko, E. J. Schofield, A. M. Jones, J. F. W. Mosselmans and A. D. Smith, Int. J. of Archit. Heritage, 2012, 6(2), 228-258.

283 M. Bertini, A. Izmer, F. Vanhaecke and E. M. Krupp, J. Anal. At. Spectrom., 2013, 28(1), 77-91.

284 Q. C. Yang, K. P. Jochum, B. Stoll, U. Weis, D. Kuzmin, M. Wiedenbeck, H. Traub and M. O. Andreae, Geostand. Geoanal. Res., 2012, 36(3), 301-313.

285 M. Crobu, A. Rossi, F. Mangolini and N. D. Spencer, Anal. Bioanal. Chem., 2012, 403(5), 1415-1432.

286 B. S. Stepanov, T. Wagner, J. Lorincik, M. Frumar, M. F. Churbanov and Y. I. Chigirinsky, Inorg. Mater., 2012, 48(6), 642-647.

287 A. I. Suchkov, A. E. Kurganova and I. D. Kovalev, Inorg. Mater., 2012, 48(8), 851-856.

288 A. Bachvarova-Nedelcheva, R. Iordanova, K. L. Kostov, S. Yordanov and V. Canev, Opt. Mater., 2012, 34(11), 1781-1787.

289 A. Agostino and R. Falcone, Glass Technol.: Eur. J. Glass Sci. Technol., Part A, 2012, 53(2), 60-64.

290 E. R. Schenk and J. R. Almirall, Forensic Sci. Int., 2012, 217(1-3), 222-228.

291 F. Alamilla, M. Calcerrada, C. Garcia-Ruiz and M. Torre, Anal. Methods, 2013, 5(5), 1178-1184.

292 M. N. C. Grainger, M. Manley-Harris and S. Coulson, J. Anal. At. Spectrom., 2012, 27(9), 1413-1422.

293 S. Kappel, S. F. Boulyga and T. Prohaska, J. Environ. Radioact., 2012, 113, 8-15.

294 J. Neeway, A. Abdelouas, B. Grambow, S. Schumacher, C. Martin, M. Kogawa, S. Utsunomiya, S. Gin and P. Frugier, J. Non-Cryst. Solids, 2012, 358(21), 2894-2905.

295 V. Soltesz, I. Vicena, M. Liska, D. Galuskova and J. M. Mattei, Glass Technol.: Eur. J. Glass Sci. Technol., Part A, 2012, 53(4), 139-145.

296 M. W. Loewen and A. J. R. Kent, J. Anal. At. Spectrom., 2012, 27(9), 1502-1508.

297 J. Czas, K. P. Jochum, B. Stoll, U. Weis, Q. C. Yang, D. E. Jacob and M. O. Andreae, Spectrochim. Acta, Part B, 2012, 78, 20-28.
298 J. I. Kimura and Q. Chang, J. Anal. At. Spectrom., 2012, 27(9), 1549-1559.

299 H. Huang, L. M. Yang and J. Liu, Appl. Opt., 2012, 51(36), 8669-8676.

300 M. Bouza, B. Fernandez, C. Gonzalez-Gago, N. Bordel, R. Pereiro and A. Sanz-Medel, Anal. Chim. Acta, 2012, 756, 30-36.

301 K. Van Hoecke, C. Catry and F. Vanhaecke, J. Pharm. Biomed. Anal., 2013, 77, 139-144.

302 J. L. Steeb, C. J. Mertz, G. Sandi, D. A. Bass, D. G. Graczyk and M. M. Goldberg, J. Radioanal. Nucl. Chem., 2012, 292(2), 757-762.

303 S. Chhillar, R. Acharya, S. Sodaye, K. Sudarshan, S. Santra, R. K. Mishra, C. P. Kaushik, R. K. Choudhury and P. K. Pujari, J. Radioanal. Nucl. Chem., 2012, 294(1), 115119.

304 N. Schibille, P. Degryse, M. O'Hea, A. Izmer, F. Vanhaecke and J. McKenzie, Archaeometry, 2012, 54, 997-1022.

305 S. Polic-Radovanovic, S. Ristic, J. Stasic and M. Trtica, J. Non-Cryst. Solids, 2012, 358(22), 3048-3056.

306 A. Detcheva, J. Hassler and R. Georgieva, Anal. Lett., 2012, 45(5-6), 603-612.

307 M. Ganio, K. Latruwe, D. Brems, P. Muchez, F. Vanhaecke and P. Degryse, J. Anal. At. Spectrom., 2012, 27(8), 13351341.

308 M. Ganio, S. Boyen, T. Fenn, R. Scott, S. Vanhoutte, D. Gimeno and P. Degryse, J. Anal. At. Spectrom., 2012, 27(5), 743-753.

309 A. Meek, J. Henderson and J. Evans, J. Anal. At. Spectrom., 2012, 27(5), 786-795.

310 I. Biron and M. Verita, J. Archaeol. Sci., 2012, 39(8), 27062713.

311 S. Cagno, M. B. Badano, F. Mathis, D. Strivay and K. Janssens, J. Archaeol. Sci., 2012, 39(7), 2191-2197.

312 D. Lesigyarski, Z. Smit, B. Zlateva-Rangelova, K. Koseva and I. Kuleff, J. Radioanal. Nucl. Chem., 2013, 295(3), 16051619.

313 Z. Smit, T. Knific, D. Jezersek and J. Istenic, Nucl. Instrum. Methods Phys. Res., 2012, 278, 8-14.

314 K. Won-in, Y. Thongkam, T. Kamwanna and P. Dararutana, J. Radioanal. Nucl. Chem., 2012, 294(2), 247-250.

315 N. Schiavon, A. Candeias, T. Ferreira, M. D. Lopes, A. Carneiro, T. Calligaro and J. Mirao, Archaeometry, 2012, 54, 974-996.

316 K. Tantrakarn, N. Kato, I. Nakai, Y. Shindo and B. Chaisuwan, Archaeometry, 2012, 54, 508-527.

317 Y. Abe, R. Harimoto, T. Kikugawa, K. Yazawa, A. Nishisaka, N. Kawai, S. Yoshimura and I. Nakai, J. Archaeol. Sci., 2012, 39(6), 1793-1808.

318 S. Liu, Q. H. Li, F. Gan, P. Zhang and J. W. Lankton, J. Archaeol. Sci., 2012, 39(7), 2128-2142.

319 R. B. Scott, A. J. Shortland, P. Degryse, M. Power, K. Domoney, S. Boyen and D. Braekmans, Glass Technol.: Eur. J. Glass Sci. Technol., Part A, 2012, 53(2), 65-73.

320 J. Hormes, A. Roy, G. L. Bovenkamp, K. Simon, C. Y. Kim, N. Borste and S. Gai, Appl. Phys. A: Mater. Sci. Process., 2013, 111(1), 91-97. 
321 S. P. Chenakin, R. P. Silvy and N. Kruse, Top. Catal., 2012, 55(11-13), 731-746.

322 W. M. Li, J. Wu, D. C. Higgins, J. Y. Choi and Z. W. Chen, ACS Catal., 2012, 2(12), 2761-2768.

323 M. B. Gawande, A. K. Rathi, P. S. Branco, T. M. Potewar, A. Velhinho, I. D. Nogueira, A. Tolstogouzov, C. A. A. Ghumman and O. Teodoro, RSC Adv., 2013, 3(11), 3611-3617.

324 M. Henry, M. Bulut, W. Vermandel, B. Sels, P. Jacobs, D. Minoux, N. Nesterenko and J. P. Dath, Appl. Catal., A, 2012, 437, 96-103.
325 C. Swalus, M. Jacquemin, C. Poleunis, P. Bertrand and P. Ruiz, Appl. Catal., B, 2012, 125, 41-50.

326 A. V. Dyachkova, T. M. Malutina, T. Y. Alekseeva and Y. A. Karpov, Inorg. Mater., 2012, 48(14), 1272-1278.

327 M. S. Paraskevas, K. Papoutsi and M. OchsenkuhnPetropoulou, Anal. Lett., 2012, 45(5-6), 539-550.

328 S. Sacmaci, S. Kartal and S. Dural, J. Braz. Chem. Soc., 2012, 23(6), 1033-1040.

329 H. Eskandari, Turk. J. Chem., 2012, 36(4), 631-643.

330 M. Li, N. S. Marinkovic and K. Sasaki, Electrocatalysis, 2012, 3(3-4), 376-385. 